\title{
Catalog for the ESPRESSO blind radial velocity exoplanet survey ${ }^{\star}$
}

\author{
S. Hojjatpanah ${ }^{1,2}$, P. Figueira ${ }^{3,1}$, N. C. Santos ${ }^{1,2}$, V. Adibekyan ${ }^{1}$, S. G. Sousa ${ }^{1}$, E. Delgado-Mena ${ }^{1}$, Y. Alibert ${ }^{4}$, \\ S. Cristiani ${ }^{5,6}$, J. I. González Hernández ${ }^{7,8}$, A. F. Lanza ${ }^{9}$, P. Di Marcantonio ${ }^{5}$, J. H. C. Martins ${ }^{1}$, G. Micela ${ }^{10}$, \\ P. Molaro ${ }^{5}$, V. Neves ${ }^{11,12}$, M. Oshagh ${ }^{13,1}$, F. Pepe ${ }^{14}$, E. Poretti ${ }^{15,16}$, B. Rojas-Ayala ${ }^{17}$, R. Rebolo ${ }^{7,8,18}$, \\ A. Suárez Mascareño ${ }^{14}$, and M. R. Zapatero Osorio ${ }^{19}$
}

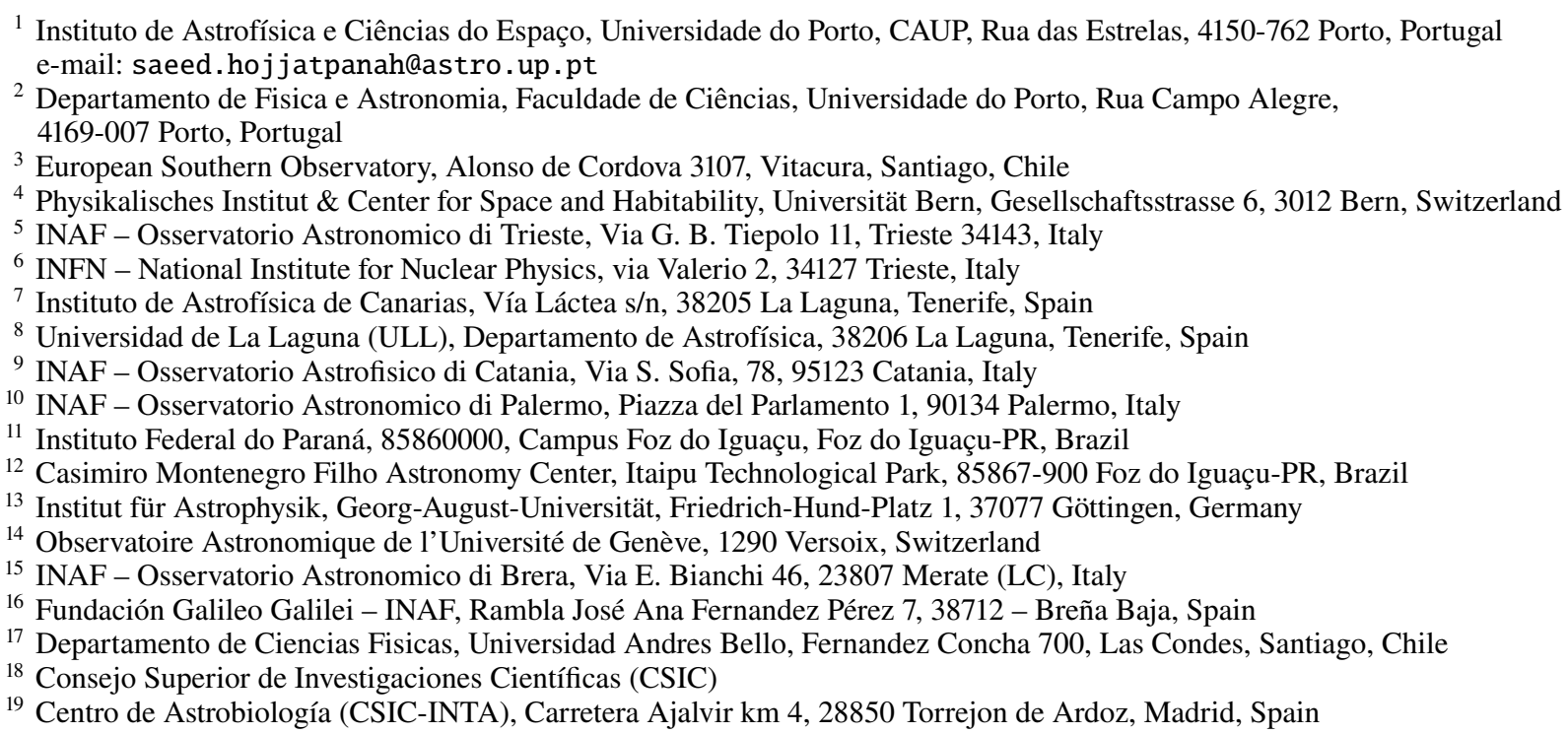

Received 27 November 2018 / Accepted 29 July 2019

\begin{abstract}
Aims. One of the main scientific drivers for ESPRESSO, Échelle SPectrograph, is the detection and characterization of Earth-class exoplanets. With this goal in mind, the ESPRESSO guaranteed time observations (GTO) Catalog identifies the best target stars for a blind search for the radial velocity (RV) signals caused by Earth-class exoplanets.

Methods. Using the most complete stellar catalogs available, we screened for the most suitable G, K, and M dwarf stars for the detection of Earth-class exoplanets with ESPRESSO. For most of the stars, we then gathered high-resolution spectra from new observations or from archival data. We used these spectra to spectroscopically investigate the existence of any stellar binaries, both bound or background stars. We derived the activity level using chromospheric activity indexes using $\log \left(R_{\mathrm{HK}}^{\prime}\right)$, as well as the projected rotational velocity $v \sin i$. For the cases where planet companions are already known, we also looked at the possibility that additional planets may exist in the host's habitable zone using dynamical arguments.

Results. We estimated the spectroscopic contamination level, $v \sin i$, activity, stellar parameters and chemical abundances for 249 of the most promising targets. Using these data, we selected 45 stars that match our criteria for detectability of a planet like Earth. The stars presented and discussed in this paper constitute the ESPRESSO GTO catalog for the RV blind search for Earth-class planets. They can also be used for any other work requiring a detailed spectroscopic characterization of stars in the solar neighborhood.
\end{abstract}

Key words. planetary systems - planets and satellites: composition - techniques: radial velocities - techniques: spectroscopic stars: abundances

\section{Introduction}

Since the discovery of 51 Peg b (Mayor \& Queloz 1995), the radial velocity $(\mathrm{RV})$ method has been extensively used to detect,

\footnotetext{
* Based on observations collected at the La Silla Observatory, ESO(Chile), with the HARPS spectrograph at the 3.6-m telescope for program 97.C - 0561 $(A)$, data obtained at the Paranal Observatory of the European Southern Observatory with UVES at VLT for program 097. $C-0561(B)$ and data obtained at the TNG telescope for program A33TAC_7, and also 95 programs. The large list is presented in Table B.1.
}

confirm, and estimate the planetary mass of exoplanets. Over the past years, hundreds of exoplanets have been discovered with the RV technique using diverse spectrographs with different levels of precision in the measurement of RVs. For instance, HARPS (High Accuracy Radial velocity Planet Searcher), on the European Southern Observatory $3.6 \mathrm{~m}$ telescope at La Silla, is one of the most successful planet-hunters. It has achieved an unprecedented precision of about $80 \mathrm{~cm} \mathrm{~s}^{-1}$ over long observing periods (Pepe et al. 2014a). After $15 \mathrm{yr}$ of intensive observations and many groundbreaking results (e.g., Santerne et al. 2018; Lovis et al. 2011; Anglada-Escudé et al. 2016; Díaz et al. 2016) 
HARPS has arguably reached its RV precision limit, and a new spectrograph was designed to be its successor: ESPRESSO (Échelle SPectrograph for Rocky Exoplanets and Stable Spectroscopic Observations) the new ultra-stable, fibre-fed, crossdispersed, high-resolution échelle spectrograph operates at the combined coudé focus of the European Southern Observatory's Very Large Telescope (VLT) (Pepe et al. 2014b; González Hernández et al. 2018).

It is important to note that ESPRESSO was designed to target several science drivers, including exoplanets, variations of fundamental constants, and spectroscopy of extra-galactic stars (Pepe et al. 2010). Among others, ESPRESSO will also allow many other problems of modern astrophysics to be tackled, including the measurement of stellar oscillations, the derivation of detailed chemical abundances in stars, as well as the study of interstellar gas.

ESPRESSO is able to use the superior light collection capabilities of ESO's VLTs and reach a RV precision of $10 \mathrm{~cm} \mathrm{~s}^{-1}$ on bright nearby stars in a matter of minutes. Such a value is crucial if we want to detect the signature of an Earth-class planet orbiting inside the habitable zone of a main-sequence, solar-type star. The expected RV modulation amplitude for the Earth around the Sun is at most about $9 \mathrm{~cm} \mathrm{~s}^{-1}$.

The main scientific driver for ESPRESSO is the measurement of high-precision RVs of $\mathrm{G}, \mathrm{K}$, and $\mathrm{M}$ dwarfs to search for rocky planets inside the habitable zone (HZ). Eighty percent of the observing nights in the guaranteed time observations (GTO) program will be dedicated to exoplanet science, having in mind the detection of rocky planets inside the HZ, the characterization of low mass planets discovered using high-precision photometric transits, and the characterization of exoplanet atmospheres. The ESPRESSO GTO catalog presented in this paper is meant to tackle the first of these cases by selecting the best candidates for detecting RV signals of low mass planets. To obtain the most precise RV measurements, several criteria should be considered to select the best targets. We defined a photon noise criterion in order to select the brightest stars. This criterion is a conservative approximation of the achievable photon noise by ESPRESSO. We also determined the activity indexes $\log \left(R_{\mathrm{HK}}^{\prime}\right)$ and $H_{\alpha}$, the projected rotational velocity $(v \sin i)$, and the signature of the spectroscopic contamination for 249 bright and nearby stars. This is a new, unique data-set that is of natural interest to a wide range of spectroscopic studies of $\mathrm{G}, \mathrm{K}$, and $\mathrm{M}$ type solar neighbors. By using focused criteria, we selected the most visible and bright stars for ESPRESSO. We also derive precise stellar parameters and chemical abundances for most of our targets.

The outline of this paper is as follows. We first describe our data and new observations in Sect. 2. We investigate the companion signature in Sect. 3. The procedure to determine activity indicator and $v \sin i$ is explained in Sects. 4 and 5, respectively. Targets with already known planets are considered in Sect. 6. We explain stellar characterization and chemical abundance in Sect. 7. We investigate the rotational period of $M$ dwarfs in Sect. 8. Our summary and conclusion are presented in Sect. 9.

\section{Observations and data}

\subsection{Target selection}

The first step to define the sample of stars was to identify the brightest $\mathrm{G}, \mathrm{K}$, and $\mathrm{M}$ stars in the sky. Since there is no comprehensive catalog for solar neighborhood $\mathrm{G}, \mathrm{K}$, and $\mathrm{M}$ dwarfs, we decided to use two different catalogs to determine initial samples. The two catalogs used were HIPPARCOS for $\mathrm{G}$ and K stars by Perryman et al. (1997), and Lépine \& Gaidos (2011) for M stars. The HIPPARCOS catalog is very probably complete down to the $\mathrm{G}$ and $\mathrm{K}$ faintest stars of interest; and Lépine \& Gaidos (2011) was selected as the most complete catalog for $\mathrm{M}$ dwarfs. This is an all-sky catalog of M-dwarfs with apparent infrared magnitude $\mathrm{J}<10$ that provides parallax measurements and photometric distance. Lépine \& Gaidos (2011) notes that the catalog is not absolutely complete, reporting completeness levels of $\approx 75 \%$ bright $\mathrm{M}$ dwarfs $(J<10)$, which cover $\approx 60 \%$ of the southern sky. However, it is the most comprehensive and suited for our goal.

At the outset, we selected all stars from the HIPPARCOS catalog with declination $<+30^{\circ}$ (i.e., well visible from Paranal). We recovered their $\mathrm{V}$ and $\mathrm{K}$ apparent magnitudes by matching HipPARCOS to the 2MASS catalog (Cutri et al. 2003). We used Eq. (6) in Casagrande et al. (2008) and Eq. (3) in Casagrande et al. (2010) for the color calibrations and estimated the stellar effective temperatures $\left(T_{\text {eff }}\right)$. We determined an approximate spectral type from $T_{\text {eff }}$ using Carroll \& Ostlie (2017). The goal was to classify the spectral type and select $\mathrm{G}, \mathrm{K}$, and $\mathrm{M}$ stars for the primary sample. We attributed spectral types based on the spectroscopic after the stellar characterizations in Sect. 7 (as seen in the Table B.1).

To the stars mentioned above we added stars with confirmed exoplanet(s) using all methods as discussed in Sect. 6. Around $22 \%$ of the known exoplanet systems have more than one planet detected corresponding to 634 multiplanet confirmed systems by November $2018^{1}$. There are also 817 multiplanet system candidates detected by NASA's Kepler spacecraft and certainly more will be identified by TESS, Transiting Exoplanet Survey Satellite, soon (Ricker et al. 2014). The high occurrence rate shows these systems are common. As an additional factor of interest for a given host, we considered the possibility of the presence of an Earth-mass planet inside the HZ of a known (i.e., confirmed) planet system (see Sect. 6).

\subsection{Cleaning by applying a photon noise criterion}

For an Earth-mass exoplanet inside the HZ to be detectable by ESPRESSO, the RV signal created by the planet should be larger than the photon noise of our measurements. To derive the location of the $\mathrm{HZ}$ around each star of the sample, we used the definition of Selsis et al. (2007), which satisfies two general conditions for HZ: (1) there should be the capability of hosting liquid water on the surface at any temperature between $273 \mathrm{~K}$ (triple point of water) and $647 \mathrm{~K}$ (critical temperature of water); and (2) the amount of $\mathrm{CO}_{2}$ in the planet's atmosphere should be abundant enough when the mean surface temperature decreases to less than $273 \mathrm{~K}$ (for more information see Selsis et al. 2007). We determined the position of the continuous HZ using the definition from Selsis et al. (2007) for the "extreme theoretical limits of the continuous habitable zone" and of an RV semi-amplitude signal $\left(K_{\max }=10 \mathrm{~cm} \mathrm{~s}^{-1}\right)$ for $1_{\sigma}$. We then computed $K_{\max }$ for an Earth-mass planet at a semi-major axis corresponding to the inner boundary of the HZ using Eq. (2.28) in Perryman (2011):

$K=28.4 \mathrm{~ms}^{-1}\left(\frac{P_{\mathrm{p}}}{1 \mathrm{yr}}\right)^{-1 / 3}\left(\frac{M_{\mathrm{p}} \sin i}{M_{\mathrm{J}}}\right)\left(\frac{M_{\star}}{M_{\odot}}\right)^{-2 / 3}$,

where $P_{\mathrm{p}}, M_{\mathrm{p}} \sin i$, are the period and minimum mass of the planet, and $M_{\star}$ is the mass of the host star. We consider the

1 https://exoplanetarchive.ipac.caltech.edu 
Table 1. RV signal semi-amplitude of one Earth-mass planet for $\mathrm{HZ}$ limits.

\begin{tabular}{cccccc}
\hline \hline $\begin{array}{c}\text { Spectral } \\
\text { type }\end{array}$ & $\begin{array}{c}\text { Mass } \\
\left(M_{\odot}\right)\end{array}$ & $\begin{array}{c}a_{\min } \\
(\mathrm{AU})\end{array}$ & $\begin{array}{c}a_{\max } \\
(\mathrm{AU})\end{array}$ & $\begin{array}{c}K_{\min } \\
\left(\mathrm{cm} \mathrm{s}^{-1}\right)\end{array}$ & $\begin{array}{c}K_{\max } \\
\left(\mathrm{cm} \mathrm{s}^{-1}\right)\end{array}$ \\
\hline G0 & 1.0 & 0.5 & 3.0 & 5.2 & 13 \\
G5 & 0.9 & 0.4 & 2.0 & 6.7 & 15 \\
K0 & 0.8 & 0.3 & 1.5 & 8.2 & 18 \\
K5 & 0.7 & 0.2 & 1.0 & 11 & 24 \\
M0 & 0.5 & 0.1 & 0.5 & 18 & 40 \\
M5 & 0.2 & 0.04 & 0.2 & 45 & 100 \\
M8 & 0.1 & 0.01 & 0.08 & 100 & 283 \\
\hline
\end{tabular}

inner boundary in order to provide the optimistic case with the maximum $K$ value.

HARPS is able to reach a precision of about $1 \mathrm{~m} \mathrm{~s}^{-1}$ in one minute on a late $\mathrm{G}$ dwarf of magnitude $V=7.5$. This project was started several years ago, much before the first ESPRESSO data were obtained. We used HARPS at the $3.6 \mathrm{~m}$ as a starting point for the photon noise calculations of ESPRESSO at the VLT. Due to the larger collecting area (i.e., mirror sized of $8.2-\mathrm{m}$ vs. 3.6-m), we assumed a gain of 1.75 magnitude for ESPRESSO relative to HARPS. Therefore we considered that ESPRESSO will have a gain of a factor of five in flux, and that the measurements would be done with a minimum integration time of $15 \mathrm{~min}$ (to average out oscillation modes Dumusque et al. 2011). We calculated the photon noise achievable by ESPRESSO by scaling it from experience using HARPS. As a consequence, the photon noise limits for $V$ can be defined by,

$V=5 \log \left(K_{\max } \mathrm{cm} \mathrm{s}^{-1}\right)+2.1$,

where $V$ is an estimation for the visual magnitude limit. By application of this formula to the $K_{\max }$ in Table 1, we estimated the following cutoff as the photon noise criteria: G0: $V<7.7$, G5: $V<8.0, \mathrm{~K} 0: V<8.4$, K5: $V<9.0$, M0: $V<10.1$, M5: $V<12.1$, and M8: $V<14.4$.

These values are compatible with the RV precision, calculated using the ESPRESSO Exposure Time Calculators, ETC ${ }^{2}$. For example, using a $\mathrm{K} 2 \mathrm{~V}$ template for a star in magnitude 8.4 and exposure time of $15 \mathrm{~min}$, we have $19 \mathrm{~cm} \mathrm{~s}^{-1}$ for the RV photon noise precision. Using the $\mathrm{M} 2 \mathrm{~V}$ template available in ETC, for a star in magnitude 12.1 and exposure time of $15 \mathrm{~min}$, we have $110 \mathrm{~cm} \mathrm{~s}^{-1}$ for the RV photon noise precision. We should point out that such an estimation for the photon noise is heavily dependent on spectral type. Most of the lines that have been used to measure RV are in the blue part of the spectrum for $G$ spectral type and in the red part for the $M$ spectral types. As such the precision is not only dependent on the visual magnitude, and here we only considered photon noise limitation (Neal \& Figueira 2019).

\subsection{Photometric variability in HIPPARcos catalog}

In the HIPPARCOS catalog, we considered large photometric variability as an exclusion criterion and discarded stars with a variability flag larger than $0.06 \mathrm{mag}$. We considered stars with HvarType flags C, M, R, $\mathrm{U}^{3}$ and also stars without any

\footnotetext{
2 https://www.eso.org/observing/etc/

3 In HIPPARCOS: HIPPARCOS-defined type of variability flag that were $\mathrm{C}, \mathrm{M}, \mathrm{R}$, and $\mathrm{U}$ (constant, micro-variable, revised color and unknown).
}

Table 2. Summary of data sources and number of spectra.

\begin{tabular}{cccccc}
\hline \hline & G and K type & M type & HARPS & HARPS-N & UVES \\
\hline Archive & 28 & 95 & 100 & 0 & 22 \\
New observation & 78 & 44 & 78 & 44 & 42 \\
Total & 106 & 139 & 178 & 44 & 64 \\
\hline
\end{tabular}

HvarType flags in the catalog. We identified a target as a single component and isolated star by using the Ncome $^{4}$ flag and by matching HIPPARCOS to the 2MASS catalog for the infrared counterpart of these stars as the closest source within $5^{\prime \prime}$ of the optical coordinates.

\subsection{Surface gravity}

In the next step we estimated the surface gravity of each star, with the goal of excluding evolved stars. Several studies have shown that for evolved stars the RV's jitter is much larger than for main sequence stars (e.g. Setiawan et al. 2004; Hatzes et al. 2005; Hekker et al. 2008; Lovis \& Mayor 2007). From Eq. (1) of Santos et al. (2004), using the mentioned quantities and the HiPPARCOS parallax, we calculated the $\log g$ of the stars from

$$
\begin{aligned}
\log \left(\frac{g}{g_{\odot}}\right)= & \log \left(\frac{M}{M_{\odot}}\right)+4 \log \left(\frac{T_{\text {eff }}}{T_{\text {eff }_{\odot}}}\right) \\
& +2 \log \pi+0.4(V+\mathrm{BC})+0.11
\end{aligned}
$$

where $V$ is the visual magnitude, $\pi$ is the parallax in arcseconds, $\mathrm{BC}$ is the bolometric correction, and $M$ is the stellar mass. Using each $T_{\text {eff }}$ we attributed an approximated mass at main-sequence, and bolometric correction to each star (Carroll \& Ostlie 2017). Due to a miscalculation in $\log g$ used in the selection process, we selected a large number of stars with low $\log g$ for further analysis. These were discarded in a later stage when a precise $\log g$ was derived from the spectra, having no impact on the final results. We applied a cutoff on $\log g>4.1 \mathrm{~cm} \mathrm{~s}^{-2}$ to exclude evolved stars from the final sample. The list of stars fulfilling all our criteria in HIPPARCOS has 187 entries, from which 15 were identified as spectroscopic binaries by Pourbaix et al. (2004), and were discarded (see Table B.5). Of the remaining 172 stars, we identified 70 stars that had been observed with HARPS or UVES at least two times with a signal-to-noise ratio $(\mathrm{S} / \mathrm{N})$ of at least 50 for $\mathrm{G}$ and $\mathrm{K}$, and 10 for $\mathrm{M}$ spectral type. The acquisition of new observations is discussed in Sect. 2.7 (see Table 2).

\subsection{Sampled M-dwarfs}

We repeated the procedure described in Sect. 2.1 for the M-dwarf catalog prepared by Lépine \& Gaidos (2011). Most of the stars in this catalog are closer than 60 parsec and are of K7 to M4 spectral type, except for a few late M-dwarfs. We searched for stars respecting the $V<14.5$ and declination $<+30^{\circ}$ criteria. We followed the same procedure while ignoring the $\log g$ restrictions, as in this dataset giants are supposed to have already been excluded (but see Sect. 2.6). By applying these criteria, we selected 114 M stars from Lépine \& Gaidos (2011).

Fifty-two of the stars selected above have high-quality data in the ESO archive, six of the stars are listed as spectroscopic binaries by Pourbaix et al. (2004) (see Table B.5) and five others were identified as binaries or as having close companions using

4 Hipparcos: Number of components in this entry. 


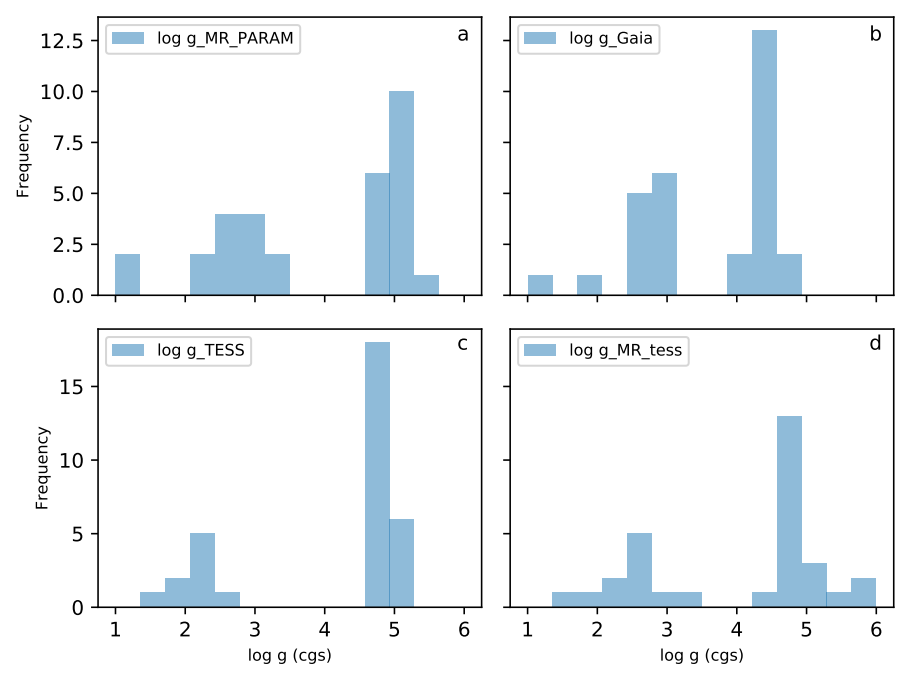

Fig. 1. Distribution of $\log g$ for cooler stars in final sample. This was completed using: (a) mass and radius derived by theoretical isochrones; (b) Gaia parallaxes; $(c)$ directly from TIC; and $(d)$ mass and radius reports in TIC.

Simbad database ${ }^{5}$ records. The remaining forty-one stars were observed (see Sect. 2.7 and Table 2).

\subsection{Screening the M-dwarfs in the sample by log $g$}

As mentioned above, the M-stars in our sample were supposed to be all dwarfs. However, to check for this assumption, we derived $\log g$ values for all the stars using the following approaches:

- $\log g$ using theoretical isochrones:

We used the theoretical isochrones described in da Silva et al. (2006) and the Padova web interface ${ }^{6}$. We then used the mass and radius derived with this method in order to determine the $\log g$ (Fig. 1a), using the formula directly:

$\log g=\log _{10}\left(\frac{G M}{R^{2}}\right)$.

- $\log g$ using Gaia parallaxes (trigonometric gravities):

After the Gaia Data Release 2 (Brown et al. 2018), we derived $\log g$ by using the Eq. (3) with Gaia parallax values (Fig. 1b). We used the mass values in $\mathrm{TIC}^{7}$ and the BC extracted using Table 1 in Torres (2010) as a function of temperature.

- $\log g$ in TESS Catalog:

After the TESS input catalog release, we identified the targets in the TIC and used the $\log g$ in the catalog (Stassun et al. 2018) (Fig. 1c). We also calculated $\log g$ by mass and radius reports in TIC and Eq. (4) (Fig. 1d).

In Fig. 1, we present the distribution of the $\log g$ for cool stars $\left(T_{\text {eff }}<3950\right)$ as derived by each method. There are ten $\operatorname{stars}^{8}$ in the Lépine \& Gaidos (2011) that are characterized as having $\log g$ $<3.5$ by all the above methods. As such, these are confidently classified as giant stars; we excluded these stars from the final

5 SIMBAD Astronomical Database: http://simbad.u-strasbg. $\mathrm{fr} /$ Simbad/: PMJ13550-2905, PMJ16554-0819, PMJ16554-0820, PMJ16589-3933N, PMJ17464+2743W.

6 http://stev.oapd.inaf.it/cgi-bin/param_1.3

7 Tess Input Catalog.

8 PMJ03219+0940, PMJ03340+1123, PMJ03377+1042, PMJ160154710, PMJ16051-0508, PMJ17173+0003, PMJ18400-1644, HIP78508, HIP79148, HIP81705. list. There are also five stars ${ }^{9}$ for which the $\log g$ have different values using the Gaia parallax and TIC if available. Therefore, these targets were flagged as potential giants and will be followed with high caution when performing RV measurements.

\subsection{Acquisition of new high-resolution observations}

High-resolution spectra for the previously described list of 122 stars for which no high-resolution high-S/N data were available, were requested in open time calls of an ESO proposal (program ID: 097.C-0561 $\left.(\mathrm{A}-\mathrm{B})^{10}\right)$, and then through a HARPS-N complementary proposal (program ID: A33TAC_ $7^{11}$ ). The instruments used were HARPS, HARPS-N (Cosentino et al. 2012) mounted on the $3.58 \mathrm{~m}$ Telescopio Nazionale Galileo at Roque de los Muchachos Observatory in La Palma (Canary Islands, Spain), and UVES (Dekker et al. 2000) spectrographs at Unit Telescope 2 of the VLT array.

Since M stars are faint in the optical, most of the M dwarfs identified as potential candidates were observed with UVES, mounted on the VLT. The choice of UVES as an instrument provides, just like HARPS, full coverage of the spectral optical domain, allowing the activity indicators to be covered. As an example, for a $V=12.0$, M 2 star we obtained a $\mathrm{S} / \mathrm{N}$ of $\sim 15$ at the $\mathrm{Ca}$ II $\mathrm{H} \& \mathrm{~K}$ regions, and $S / N \sim 100$ at $600 \mathrm{~nm}$ central wavelength.

The final sample consists of stars observed from 3 to 8 May 2016 using HARPS, UVES UT2-Kueyen between April-July 2016, and using HARPS-N, between July-September 2016. For each target, at least two spectra were collected on different nights. In Table 2, a brief overview of the observations is presented. The average $\mathrm{S} / \mathrm{N}$ for spectra obtained by HARPS and HARPS-N is 210 and by UVES is 51 at mid-wavelength (around $550 \mathrm{~nm})$.

\subsection{Data reduction and cross-correlation function derivation}

For HARPS and UVES, we collected all reduced, raw, and associated calibration-reduction spectra taken each night using the ESO archives ${ }^{12}$. We performed the same data collection procedure for the 122 targets that were already observed at least twice using HARPS and UVES spectrographs, above the threshold of 210 for HARPS and HARPS-N and 51 for UVES. For HARPS-N and HARPS spectra data reduction, we used the latest version of the HARPS-N pipeline (DRS V3.8) using the Cross-Correlation Function, CCF technique with a template of G2, K5, and M2 masks (Pepe et al. 2002; Baranne et al. 1996). This pipeline provides the CCF's full width at half maximum (FWHM), CCF's bisector span inverse slope, and the Ca II activity index $\mathrm{S}$ and $\log \left(R_{\mathrm{HK}}^{\prime}\right)$ for $\mathrm{F}, \mathrm{G}$, and $\mathrm{K}$ stars.

In order to evaluate the presence of a second set of spectroscopic lines, we need to calculate the CCF in a wide window (see Sect. 3). We used the following procedure to calculate the CCFs in a range of $200 \mathrm{~km} \mathrm{~s}^{-1}$ to ensure a contaminant CCF peak would not remain unnoticed.

The UVES data were reduced by a custom-made Python script that interfaces directly with ESO's CPL (Common

\footnotetext{
9 PMJ15357-2812, PMJ15543-3536, PMJ16046-5521, PMJ16118-3331, PMJ19473-2424.

10 PI: P. Figueira.

11 PI: P. Molaro.

12 https://www . eso.org/sci/observing/phase3/data_ streams.html
} 


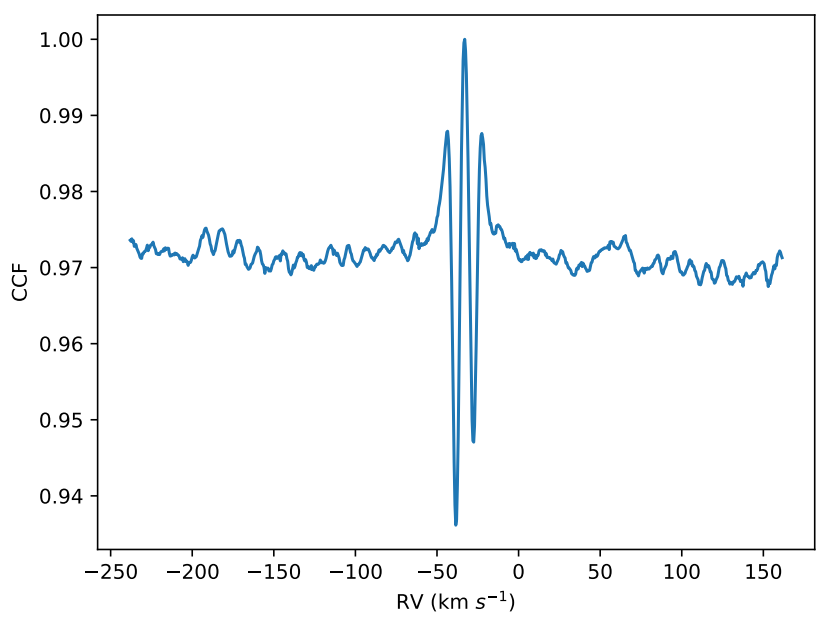

Fig. 2. Example CCF of star with two significant peaks.

Pipeline Library) data reduction recipes for the UVES spectrograph (Martins \& Figueira 2017). This method permits two different approaches: i) run the red chain recipe that performs the whole reduction; and ii) perform a step-by-step data reduction using the individual data reduction recipes. We selected the latter approach as it allows finer control over the data reduction process.

It should be noted that when performing the flat-fielding of the science raw spectra, the UVES reduction recipes will remove the instrumental profile (i.e., blaze) information from the spectra. This means that the reduced spectra loses the information about the real flux, not allowing us to estimate the noise level at each wavelength. Due to this effect, the correction of the blaze function will give incorrect weights to the spectral lines closer to the edges when computing the CCF. To tackle this issue, we used the extracted, reduced, master-flat spectra for each order to reconstruct the blaze function, meaning the instrumental profile of the instrument on each order. These were constructed by performing a moving average over the extracted flat field for each order with a window of 100 pixels in the spectral space. This allows us to recover the instrumental profile, by smoothing the flat function over a 100-pixel region in the spectrum space to discard local defects (e.g., bad pixels). Each twodimensional spectrum was then multiplied by the reconstructed blaze function to recalculate the spectra with the real flux.

To compute the CCF of each individual spectrum, we used the spectral masks from the HARPS DRS (Mayor et al. 2003). It should be noted that although they were originally built to be used with HARPS data, the wavelength coverage of both instruments is similar enough ([450-750]nm for UVES, against [378-691]nm for HARPS) that the same masks can be used. For each individual spectrum order and a given radial velocity $\mathrm{RV}_{\mathrm{i}}$, our implementation of the CCF is mathematically defined by

$\mathrm{CCF}_{\text {order }}\left(\mathrm{RV}_{\mathrm{i}}\right)=\sum_{\text {lines,order }}\left(d_{\text {line }} \times \sum_{\text {pixels }}\left(f_{\text {line,pix }} F_{\text {line,pix }}\right)\right)$,

where $d_{\text {line }}$ is the depth of the selected line (from the binary mask); pixels represents the list of spectral pixels covered by the selected line; $f_{\text {line,pix }}$ the fraction of the pixel that falls on the line; and $F_{\text {line,pix }}$ the flux of the line for each pixel (from the spectrum). Note that the line parameter is dependent on $R V_{i}$, as it refers to the spectral line wavelength position for the given $R V_{i}$.
The CCF value is then computed over a RV range centered on the expected RV of the system, yielding a plot such as the one presented in Fig. 2.

\section{Signature of stellar companions in the CCF}

One of the goals of this study is to identify stars that are binaries or affected by the presence of a background star inside the fibre. For $\mathrm{G}$ and $\mathrm{K}$ type stars the presence of a contaminator can create a systematic RV signal of $10 \mathrm{~cm} \mathrm{~s}^{-1}$ even in the extreme case when the difference in magnitude is of ten; this parasite signal can be larger than $1 \mathrm{~m} \mathrm{~s}^{-1}$ if the difference in magnitude is less than eight (Cunha et al. 2013). For M type stars, a difference of magnitude between target and contaminant of eight, creates an effect of $10 \mathrm{~cm} \mathrm{~s}^{-1}$ and a difference of magnitude between target and contaminant of six leads to an effect of $1 \mathrm{~m} \mathrm{~s}^{-1}$ (Cunha et al. 2013). As such, a careful examination should be made to search for hints of companions.

There are several methods that can be used to check if the star has any detectable contaminations. Here we investigate using the CCF. The goal is to determine CCF's pollution induced by a second star in the sky, close enough to contribute with light to the total collected in the fiber. This companion can be gravitationally bound or fortuitously aligned with the star. If the companion or companions exist and are bright enough, the presence of a second set of lines can create either a secondary correlation peak, if well-separated in RV from the target, or a change in the CCF line profile, if blended with the target. After calculating the CCFs in a wide window (200 $\mathrm{km} \mathrm{s}^{-1}$ ), we compared the different CCFs obtained on each star for any anomaly in its usually "Gaussian shape" as well as of variability in its profile. In the first approach, we searched for the characteristic double peak signature, and identified six targets, five of which are known binaries with significant CCF contamination. We considered a Gaussian-like shape with a CCF depth of at least $10 \%$ (an admittedly arbitrary value) to be a secondary peak. In Fig. 2, it is possible to see an example of double-line CCFs due to spectroscopic contamination. We include these targets with a signature of binary nature with "Y+" flag in Table B.1.

After this visual inspection procedure, we applied a more homogeneous criterion to identify stars on which a second component was present. We followed the method of Bouchy et al. (2016): after the derivation of CCFs, we shifted the reduced CCFs to a common RV value and mutually subtracted them. We then searched if there was a significant peak in the CCF residuals (see Fig. 3). The criteria for the absence of an additional peak is defined by

$$
\sqrt{\left(S / N_{1}\right)^{2}+\left(S / N_{2}\right)^{2}}<\frac{1}{\sigma_{\mathrm{RMS}}} \text {. }
$$

Here the $S / N_{1}$ and $S / N_{2}$ are the median order signal-to-noise ratios of the CCFs, which are available in the reduced FITS headers; and $\sigma_{\mathrm{RMS}}$ is the root mean square, RMS of the residual of the CCF difference. If the above condition is not met, then this means there is an additional source of scatter in the data. Equation (6) provides a systematic way of searching for the secondary peak on CCFs affected by contamination, provided we have at least two spectra. Stars for which our analysis satisfies the above condition are flagged with " $\mathrm{N}$ ", otherwise the target contamination flag is set as "Y". The results are presented in Table B.1.

In most cases, we did not find any significant hint of companion. Twenty-one stars have a companion and were discarded according to this criteria. 

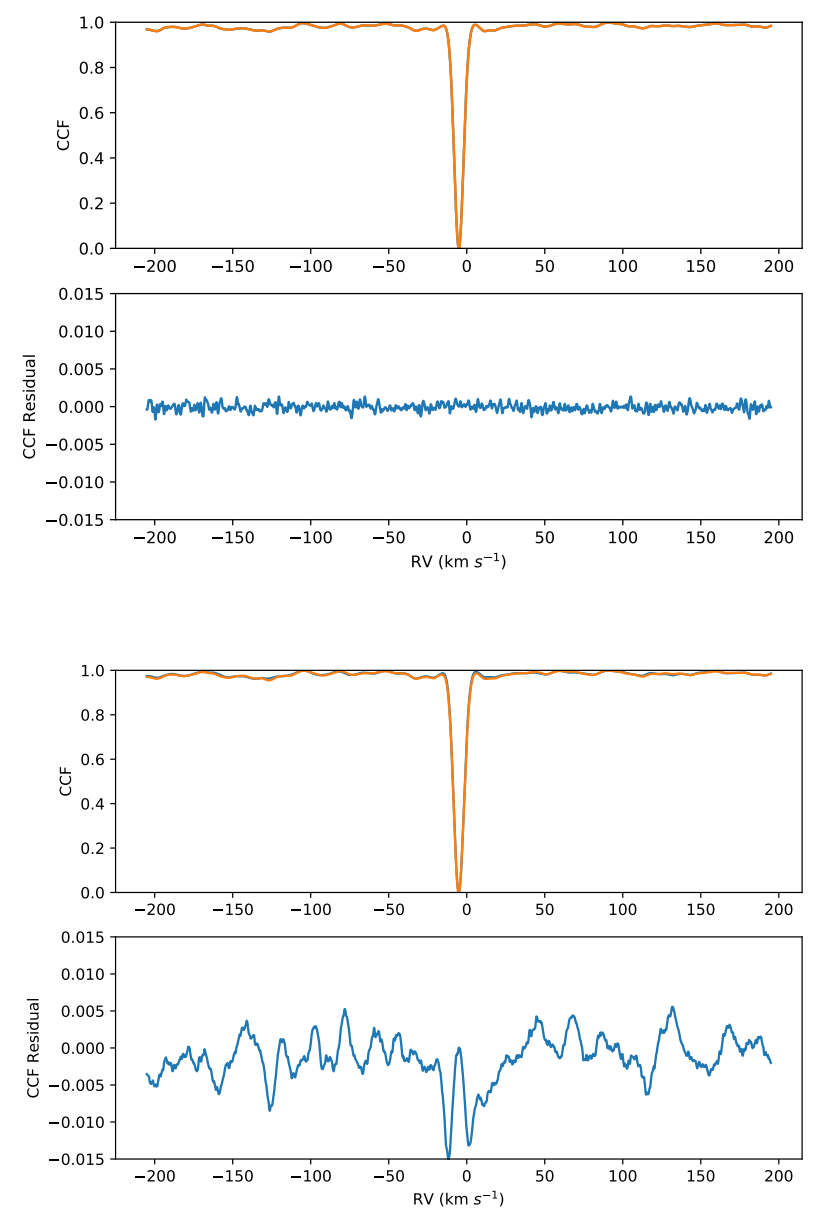

Fig. 3. Two examples of normalized CCFs and residuals of stars. Upper panels: without signs of contamination. Bottom panels: with hints of contamination.

\section{Chromospheric activity indicator $\log \left(R_{\mathrm{HK}}^{\prime}\right)$}

Stellar activity related phenomena are known to produce significant RV signals. These can both induce noise in our data or produce coherent signals that can mimic the signature of an orbiting planet. Stellar active regions (e.g., dark spots and bright faculae) produce RV signals at the level of exoplanet orbital signals when they combine with stellar rotation. Stellar active regions also deform the $\mathrm{CCF}$ profile. As a consequence, they generate parasite RV signals (Meunier et al. 2010; Dumusque et al. 2014; Oshagh et al. 2017). As such, it is mandatory to track and characterize activity signals in order to disentangle the real exoplanet RV signal (e.g., Hatzes 2013a,b; Queloz et al. 2001; Santos et al. 2014; Robertson et al. 2014; Suárez Mascareño et al. 2017, 2018).

To clear our sample from active stars, we used an activity indicator, $\log \left(R_{\mathrm{HK}}^{\prime}\right)$. We started by determining the $\mathrm{S}$-index. This is a well-known quantity used for the first time in the longterm observations in the Mount Wilson stellar activity program (Vaughan et al. 1978; Duncan et al. 1991).

We used $\mathrm{Ca}$ II $\mathrm{H} \& \mathrm{~K}$ lines and $R \& V$ continuum band-passes for $S$-index determination,

$S=\alpha \frac{N_{H}+N_{K}}{N_{R}+N_{V}}$.

The $S$-index measures the flux in the core of the line, normalized to the continuum band-passes. In this equation, $N_{H}, N_{K}$,
$N_{R}$ and $N_{V}$ are the total flux in each band-pass and $\alpha$ is a calibration constant usually adjusted at 2.30 (Duncan et al. 1991). The $1.09 \AA$ wide bands centered at 3933.664 and $3968.470 \AA$ were used for $K \& H$ respectively. For $V \& R$ we used the $20 \AA$ wide bands centered at 3901.070 and $4001.070 \AA$, respectively.

To compare different stars' activity, we need to remove the effect of the photospheric contribution and perform a normalization depending on the color index $(B-V)$ that was introduced. The $\log \left(R_{\mathrm{HK}}^{\prime}\right)$ has been defined by Noyes et al. (1984)

$R_{\mathrm{HK}}^{\prime}=1.34 \times 10^{-4} \times C_{\mathrm{cf}}(B-V) \times S-R_{\mathrm{phot}}(B-V)$,

where $C_{\mathrm{cf}}(B-V)$ is the conversion factor (Noyes et al. 1984; Middelkoop 1982). To derive $C_{\mathrm{cf}}(B-V)$ we used the recent work of Suárez Mascareño et al. (2015), who calibrated these values in the range of $\mathrm{B}-\mathrm{V}$ between 0.4 and 1.9:

$$
\begin{aligned}
\log _{10} C_{\mathrm{cf}}= & 0.668-1.270 \times B-V+0.645 \times B-V^{2} \\
& -0.443 \times(B-V)^{3} .
\end{aligned}
$$

Suárez Mascareño et al. (2015) call photospheric contribution to the $H$ and $K$ bandpasses $R_{\text {phot }}$ and we used the calibration defined as

$R_{\text {phot }}=1.48 \times 10^{-4} \times \exp [-4.3658 \times(B-V)]$.

Using these equations and measuring the fluxes in our spectra, we determined the $S$-index and $\log \left(R_{\mathrm{HK}}^{\prime}\right)$. The HARPS and HARPS-N DRS measure the $S$-index and convert this into the chromospheric activity indicator $\log \left(R_{\mathrm{HK}}^{\prime}\right)$. Both pipelines need $B-V$ and guess RV as input parameters. We used the $B-V$ values from the catalog data (e.g., HIPPARCOS) and the extracted RV from the CCFs. For HARPS-N, we used YAbI ${ }^{13}$ a web based access to the pipeline of HARPS-N. The pipeline provided the calibrated $\mathrm{S}$-index and $\log \left(R_{\mathrm{HK}}^{\prime}\right)$ for each spectrum in a similar way to HARPS.

For the stars observed with the UVES spectrograph, we corrected the RV shift using the RV's determined by the CCFs and then calculated the $\log \left(R_{\mathrm{HK}}^{\prime}\right)$.

We identified 43 stars with chromospheric activity higher than -4.80 dex, which usually is considered as the threshold for active stars (e.g., Vaughan \& Preston 1980). On the other hand, stars less active than this value are usually seen as stars with solar-like activity. We removed those active stars from our final sample. The derived activity values for all the stars are presented in Table B.1 and illustrated in Fig. 4.

\section{Projected rotational velocity $(v \sin i)$}

Another important parameter affecting RV precision is the projected rotational velocity of the host star $(v \sin i)$. Assuming, for simplicity, that the spectral lines are resolved and have a Gaussian shape, RV precision is inversely proportional to the square root of the FWHM of the lines (e.g., Bouchy et al. 2001; Figueira 2018). For a non-resolved spectral line, $\sigma_{\mathrm{RV}} \propto$ $(v \sin i)^{1.5}$, if the other broadening mechanisms can be neglected in comparison to the rotational broadening.

We estimated the $v \sin i$ using the FWHM of the CCF and $B-V$ color and following the procedure described by several studies (e.g., Santos et al. 2002; Maldonado et al. 2017, with references therein) for HARPS data. We extended the calibration and used the stars that were observed with both HARPS and HARPS-N. Knowing that the instruments are very similar in design and in particular in resolution, a linear relationship

${ }^{13}$ http://ia2.oats.inaf.it 


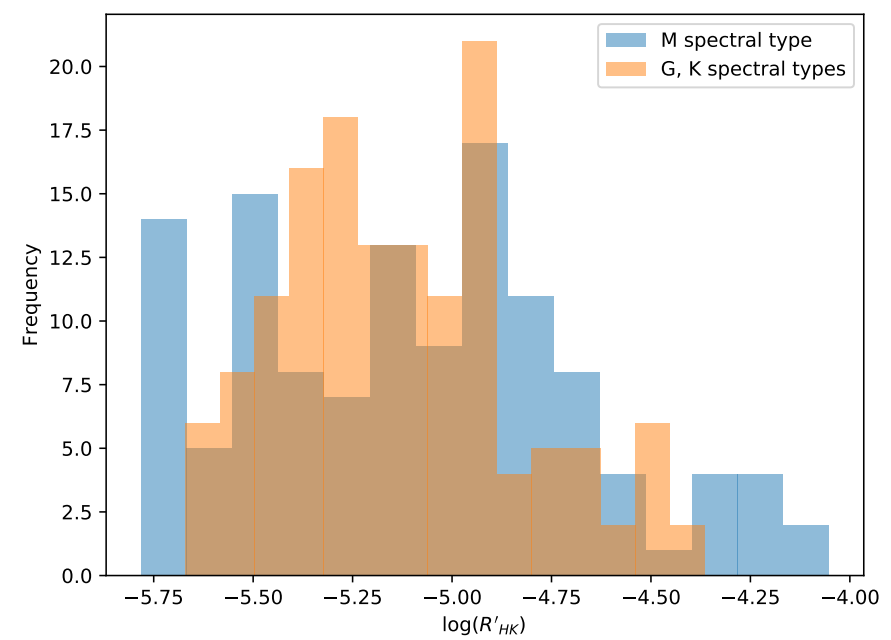

Fig. 4. Derived $\log \left(R_{\mathrm{HK}}^{\prime}\right)$ distribution for star sample.

was defined between the width of the CCF as measured by the two instruments. For CCF parameters like FWHM, we used the values produced by the previously mentioned standard pipelines of the two instruments. This allowed us to calculate the $v \sin i$ on HARPS-N for each FWHM. This calibration was performed independently for different masks corresponding to different spectral types (see Fig. 5). We followed the same procedure for UVES spectra as well. For the UVES spectra, we derived the width of the CCF and its associated uncertainty by using the tool provided by Martins \& Figueira (2017), see Sect. 2.8. We used a sample of stars in common between HARPS and UVES in order to derive $v \sin i$ for the stars observed with UVES.

In Fig. 6, we plot the distribution of the $v \sin i$ measurements. The uncertainties on $v$ sin $i$ are derived by error propagation. We considered uncertainties on the $\mathrm{CCF}$ width for non-rotating stars and on the observed CCF width (Maldonado et al. 2017). For stars with more than one observation, we considered the standard deviation of the observed CCF width and the photon noise on the CCF in the error propagation equations. The mean value of the final uncertainties is of $0.47 \mathrm{~km} \mathrm{~s}^{-1}$, which is compatible with the work of Browning et al. (2010) and Maldonado et al. (2017). Uncertainties coming from simple error propagation are by construction underestimations and the method needs a deeper analysis on the uncertainties and dependencies on both other astrophysical parameters as well as the systematic errors coming from the simplified model applied to link the $v$ sin $i$ to the FWHM of the CCF (Maldonado et al. 2017; Browning et al. 2010; Reiners et al. 2018, and references therein). However, it is enough to detect the high rotation velocity stars as it is the aim of the paper. The results for $v \sin i$ are presented in Table B.1. We reported values the $v \sin i<2.0 \mathrm{~km} \mathrm{~s}^{-1}$ as " $<2.0 \mathrm{~km} \mathrm{~s}^{-1}$ " because of the limited instrumental resolution. The FWHM of a spectrograph with $R=100000$ corresponds to $\sim 2.0 \mathrm{~km} \mathrm{~s}^{-1}$. As such, one cannot measure rotational velocity values smaller than this value. Most of the targets have low projected rotational velocity except three stars. Twenty-three targets were identified as having a high rotational velocity with $v \sin i>5.0 \mathrm{~km} \mathrm{~s}^{-1}$ and were removed from the final sample.

\section{Complementary list for the known exoplanet systems}

On top of the stars discussed above, we decided to give particular attention to the stars with known planets. In particular, we
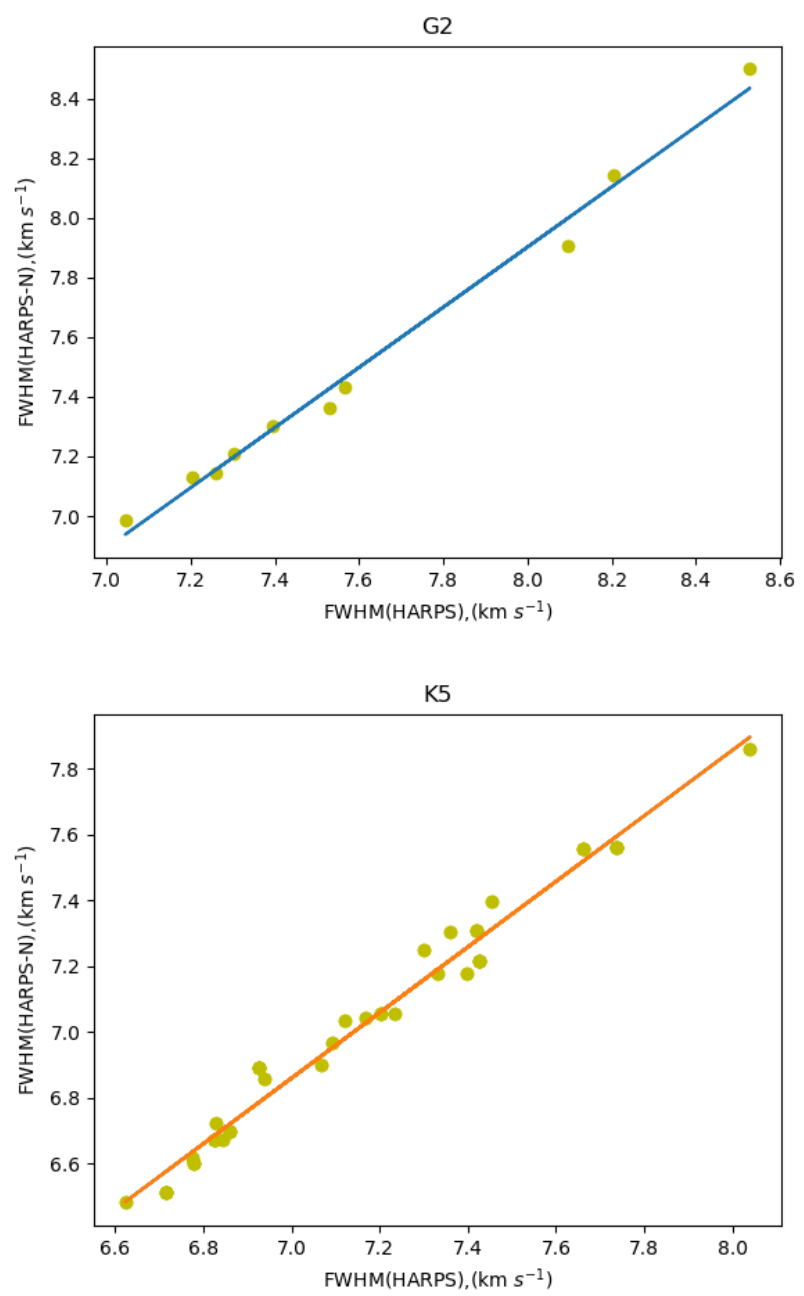

Fig. 5. Calibration of FWHM examples in order to derive $v \sin i$ for common stars sampled in HARPS and HARPS-N data.

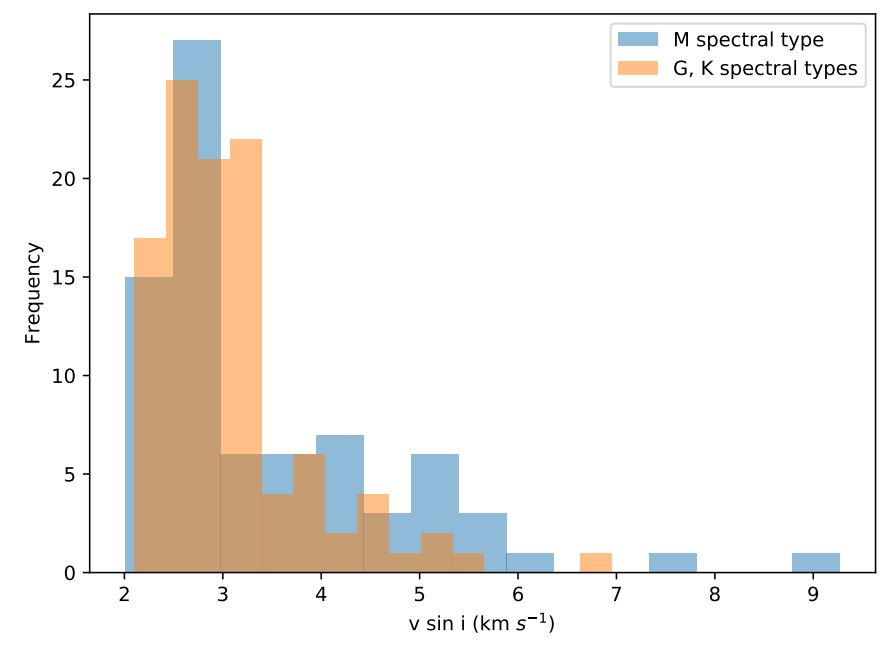

Fig. 6. Derived $v \sin i$ distribution for star sample.

looked for systems that both satisfy our previous criteria and that could host an additional Earth-mass planet in the habitable zone. We applied the photon noise criteria and visibility on the known $\mathrm{G}, \mathrm{K}$, and $\mathrm{M}$ spectral type hosting exoplanets. As a source of known exoplanets we used the Exoplanet Encyclopaedia ${ }^{14}$ and

14 http://www. exoplanet. eu/catalog/ 
Table 3. Nineteen known exoplanet systems with free stable orbit in $\mathrm{HZ}(\mathrm{C}=$ satisfy with circular orbits, EC: satisfy considering eccentric orbits).

\begin{tabular}{|c|c|c|c|c|c|c|c|c|}
\hline Star name & $T_{\text {eff }}(\mathrm{K})$ & {$[\mathrm{Fe} / \mathrm{H}]$} & $\log g\left(\mathrm{~cm} \mathrm{~s}^{-2}\right)$ & Spectral type & Period HZ (day) & $K\left(\mathrm{~cm} \mathrm{~s}^{-1}\right)$ & Stability & $\log \left(R_{\mathrm{HK}}^{\prime}\right)$ and [data sources] \\
\hline HIP43587 & $5279 \pm 62$ & $0.33 \pm 0.07$ & $4.37 \pm 0.18$ & G8 & 130.43 & 13 & $\mathrm{C}$ & $-5.070 \pm 0.012[7]$ \\
\hline HIP98767 & $5584 \pm 36$ & $0.24 \pm 0.05$ & $4.37 \pm 0.11$ & G8 & 179.46 & 12 & $\mathrm{C}$ & $-5.09[2]$ \\
\hline HIP57443 & $5629 \pm 29$ & $-0.29 \pm 0.02$ & $4.44 \pm 0.03$ & G8 & 198.01 & 12 & $\mathrm{C}$ & $-4.944 \pm 0.010[5]$ \\
\hline HIP64924 & $5577 \pm 33$ & $0.01 \pm 0.05$ & $4.34 \pm 0.11$ & G8 & 179.06 & 12 & $\mathrm{C}$ & $-4.920 \pm 0.014[1]$ \\
\hline HIP80337 & $5858 \pm 18$ & $0.03 \pm 0.01$ & $4.50 \pm 0.03$ & G2 & 225.18 & 10 & $\mathrm{C}$ & $4.52[2]$ \\
\hline HIP113357 & $5804 \pm 36$ & $0.20 \pm 0.05$ & $4.42 \pm 0.07$ & G5 & 203.02 & 10 & $\mathrm{EC}$ & $-4.963 \pm 0.042[3]$ \\
\hline HIP86796 & $5798 \pm 33$ & $0.32 \pm 0.04$ & $4.33 \pm 0.08$ & G5 & 209.91 & 10 & $\mathrm{C}$ & $-5.000 \pm 0.032[4]$ \\
\hline HIP40693 & $5402 \pm 28$ & $-0.06 \pm 0.02$ & $4.40 \pm 0.04$ & K0 & 158.35 & 13 & $\mathrm{EC}$ & $-4.898 \pm 0.250[4]$ \\
\hline HIP8102 & $5310 \pm 17$ & $-0.52 \pm 0.01$ & $4.46 \pm 0.03$ & K0 & 187.37 & 13 & $\mathrm{C}$ & $-4.886 \pm 0.202[8]$ \\
\hline HIP15510 & $5401 \pm 17$ & $-0.40 \pm 0.01$ & $4.40 \pm 0.03$ & K0 & 177.95 & 14 & $\mathrm{EC}$ & $-4.915 \pm 0.016[9]$ \\
\hline HIP99825 & $5099 \pm 65$ & $-0.30 \pm 0.04$ & $4.43 \pm 0.13$ & K0 & 135.45 & 14 & $\mathrm{C}$ & $-4.893 \pm 0.060[10]$ \\
\hline HIP3093 & $5182 \pm 79$ & $0.12 \pm 0.05$ & $4.40 \pm 0.16$ & K0 & 135.5 & 14 & $\mathrm{C}$ & $-5.02[2]$ \\
\hline HIP16537 & $5049 \pm 48$ & $-0.15 \pm 0.03$ & $4.45 \pm 0.09$ & $\mathrm{~K} 1$ & 126.01 & 15 & $\mathrm{C}$ & $-4.440 \pm 0.021[1]$ \\
\hline HIP10138 & $5114 \pm 61$ & $-0.29 \pm 0.04$ & $4.55 \pm 0.13$ & $\mathrm{~K} 1$ & 136.18 & 15 & $\mathrm{EC}$ & $-4.702 \pm 0.120[11]$ \\
\hline HIP83043 & $3519 \pm 150$ & $-0.04 \pm 0.10$ & $4.76 \pm 0.12$ & M1 & 27.79 & 32 & $\mathrm{C}$ & $-5.016[2]$ \\
\hline HIP24186 & $3550 \pm 50$ & -0.89 & - & M1 & 33.4 & 46 & $\mathrm{EC}$ & $-5.882 \pm 0.051[6]$ \\
\hline HIP85523 & $3334 \pm 110$ & $-0.23 \pm 0.09$ & $4.92 \pm 0.21$ & M2 & 31.43 & 41 & $\mathrm{EC}$ & $-4.967 \pm 0.110[6]$ \\
\hline HIP106440 & $3446 \pm 110$ & $-0.17 \pm 0.09$ & $4.83 \pm 0.15$ & M3 & 24.24 & 38 & $\mathrm{EC}$ & $-5.323 \pm 0.030[12]$ \\
\hline HIP70890 & $3050 \pm 100$ & -0.03 & 5.0 & M6 & 6.54 & 140 & $\mathrm{C}$ & $-4.963 \pm 0.009[13]$ \\
\hline
\end{tabular}

Notes. [1]: 192.C-0852(A), [2]: NASA Exoplanet Data Archive, [3]: 091.C-0271(A), [4]: 183.C-0972(A), [5]: (Gomes da Silva et al. 2014), [6]: 072.C-0488(E), [7]: 288.C-5010(A), [8]: 093.C-0062(A), [9]: 096.C-0053(A), [10]: 097.C-0021(A), [11]: 60.A-9700(G), [12]: 198.C-0838(A), [13]: 191.C-0505(A).

NASA Exoplanet Data Archive ${ }^{15}$. For the stellar parameters we used the SWEET-CAT (Santos et al. 2013; Andreasen et al. 2017) to exclude giants $\left(\log g<4.1 \mathrm{~cm} \mathrm{~s}^{-2}\right)$ and select the stars of $\mathrm{G}$, $\mathrm{K}$, and $\mathrm{M}$ spectral types. For each confirmed exoplanet system, we determined the HZ defined by Selsis et al. (2007). Then we investigated the stability of an Earth-mass planet inside $\mathrm{HZ}$ using Hill-stability for circular orbits as well as orbits with the given eccentricity in the exoplanets catalogs. For these stars we did not apply activity criteria. We then applied the Eq. (24) from Gladman (1993) as stability criterion

$\frac{a_{1}-a_{2}}{a_{1}}>2.40\left(\mu_{1}+\mu_{2}\right)^{1 / 3}$,

to ten different orbits within the HZ (in regular steps of orbital separation). Here $\mu_{i}=m_{i} / m_{\star}, m_{i}$, and $m_{\star}$ are the mass of planets and star, $a_{i}$ is a semi-major axis, and $i=1,2$ for the two planets respectively. For each of them we checked the stability condition for a potential Earth-mass planet in a circular orbit. Our goal was to select and retain only those systems for which at least one of the tested orbits is stable.

On the other hand, for systems with planets in eccentric orbits we used Eq. (21) from Gladman (1993),

$\left(\mu_{1}+\mu_{2} \frac{a_{1}}{a_{2}}\right)\left(\mu_{1} \gamma_{1}+\mu_{2} \gamma_{2} \sqrt{\frac{a_{2}}{a_{1}}}\right)^{2}>\alpha^{3}+3^{4 / 3} \mu_{1} \mu_{2} \alpha^{5 / 3}$,

where $\gamma_{i}=\sqrt{1-e^{2}}, \alpha=\mu_{1}+\mu_{2}$ and $e_{i}$ is the eccentricity. For the systems with more than one planet, each pair of planets was considered. By using the stable-orbits in the HZ of the system, we determined the Earth-mass planet orbital period in the innermost stable orbit in the most optimistic HZ using the generalized

15 https://exoplanetarchive.ipac.caltech.edu/index.html
Kepler third law:

$P / 1 \mathrm{yr}=\sqrt{\frac{a^{3} / \mathrm{AU}}{M_{\star} / M_{\odot}}}$.

Then we estimated the semi-amplitude for RV variation (K) using Eq. (1). Table 3 lists the parameters for the known exoplanet systems with at least one stable orbit. However using Hill's criterion can be risky because it is valid only for coplanar orbits (Gladman 1993) and ignores the possibility of the existence of stable islands due to the secular resonances. Therefore we did not exclude any target from the our list based on this criterion. Further comprehensive analysis of each individual system is required to reach any firm conclusion.

\section{Stellar characterization and chemical abundance}

Stellar parameters are not only crucial for accurate characterization of exoplanets but there is also increasing evidence that the characteristics of exoplanets are correlated to several host stars' properties (e.g., Adibekyan et al. 2016; Mayor et al. 2004; Fischer \& Valenti 2005; Reffert et al. 2015). In recent studies, chemical abundance has provided useful constraints for planet structure and composition, and to investigate planet formation theories (e.g., Santos et al. 2017). For this aim, we derived stellar parameters $\left(T_{\text {eff }}\right.$ : effective temperature, $[\mathrm{Fe} / \mathrm{H}]$ : metallicity, $\log g$ : surface gravity, and $\xi_{\mathrm{t}}$ : microturbulence) and chemical abundances for all of the stars in the sample.

The stellar parameters and chemical abundances for $\mathrm{G}$ and $\mathrm{K}$ dwarfs are determined with the procedure described in e.g. Sousa (2014); Adibekyan et al. (2012c). In short, we first automatically measured the equivalent widths (EWs) of the spectral lines using the Automatic Routine for line Equivalent widths in 


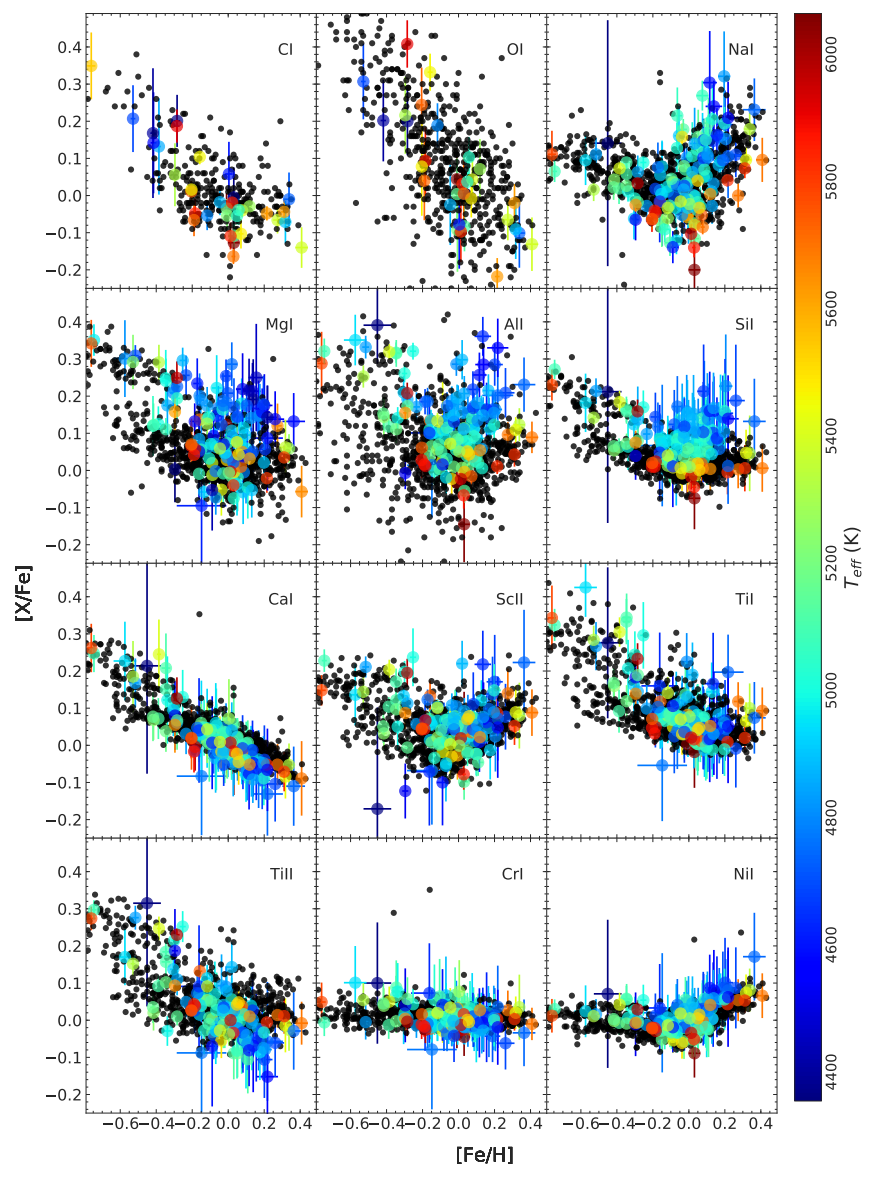

Fig. 7. Abundance ratio $[\mathrm{X} / \mathrm{Fe}]$ against stellar metallicity for current sample (color-coded by $T_{\text {eff }}$ ) and for HARPS-sampled field stars (black).

stellar Spectra, ARES v2 code (Sousa et al. 2015) ${ }^{16}$. For the more problematic elements we performed careful visual inspection of the determination of EWs. Then the spectroscopic parameters and chemical abundances were derived using the classical curveof-growth analysis method forcing excitation and ionization equilibrium under assumption of local thermodynamic equilibrium (LTE). We used the grid of the ATLAS9 plane-parallel model of atmospheres (Kurucz 1993) and the 2014 version of MOOG $^{17}$ radiative transfer code (Sneden 1973). The uncertainties of the stellar parameter and chemical abundances are also derived as in the references above. It is important to note that they represent the internal precision of the technique and not the absolute accuracies that can be significantly worse due to several factors as discussed in Hinkel et al. (e.g. 2016); Jofré et al. (e.g. 2017).

Abundances of the volatile elements, $\mathrm{C}$ and $\mathrm{O}$, were only derived for a subsample of the stars and following the method of Delgado Mena et al. (2010); Bertran de Lis et al. (2015). As discussed in those works the derivation of abundances for these elements with the EW method and the lines employed here is only possible for unevolved stars with $T_{\text {eff }} \gtrsim 5100 \mathrm{~K}$. For Carbon, we measured the EWs of two atomic lines with ARES v2 code but under a visual inspection since the line at $5052 \AA$ gets weaker as $T_{\text {eff }}$ decreases. Given that the very few oxygen lines

\footnotetext{
16 The last version of ARES code (ARES v2) can be downloaded at http://www . astro.up.pt/ sousasag/ares

17 The source code of MOOG can be downloaded at http://www . as . utexas. edu/ chris/moog.html
}

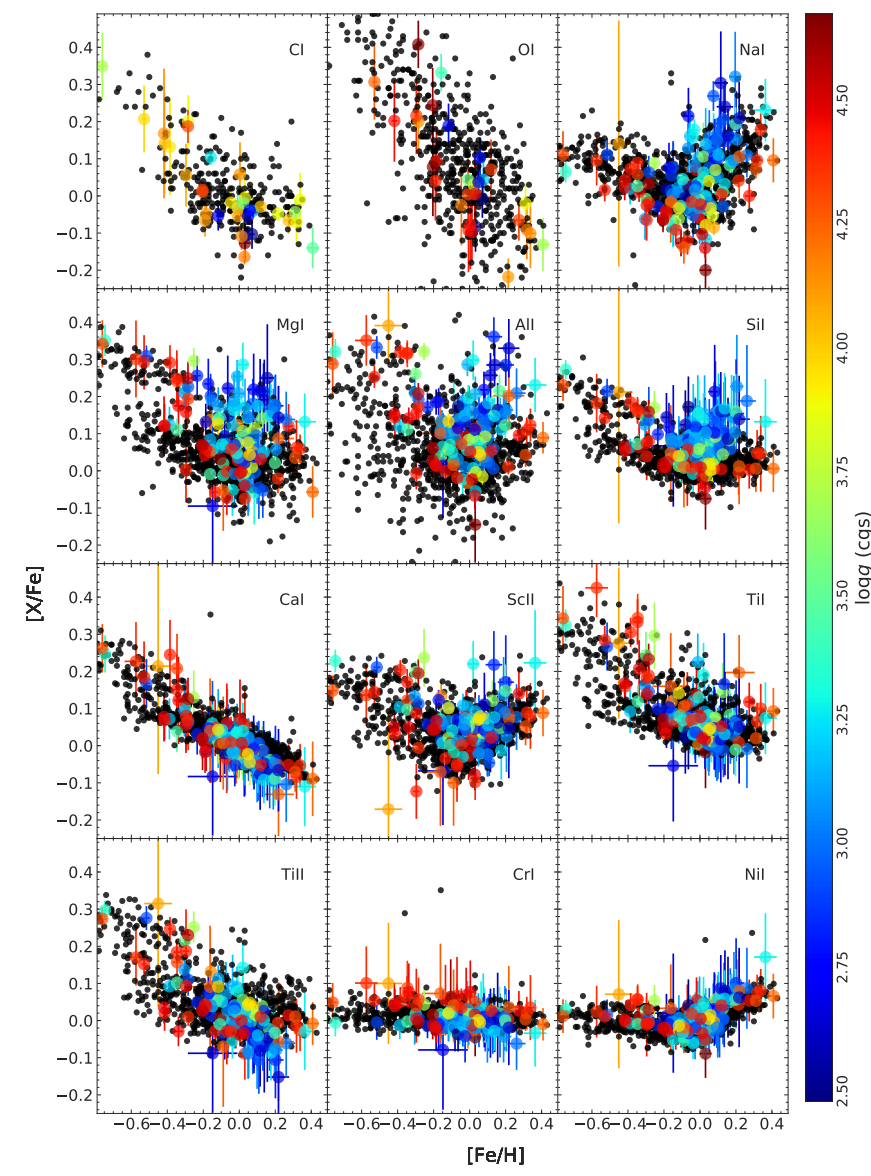

Fig. 8. Abundance ratio $[\mathrm{X} / \mathrm{Fe}]$ against stellar effective temperature for current sample (color-coded by $\log g$ ) and for HARPS-sampled field stars (black).

may be strongly blended, we choose to manually measure them with the task splot in Image Reduction and Analysis Facility, IRAF. The line at $6158 \AA$ gets very weak for cooler stars and the forbidden line at $6300 \AA$ is blended with Ni and CN lines (which become stronger as $T_{\text {eff }}$ decreases). Then the abundances were derived using the 2014 version of MOOG as they were for the other elements.

In Figs. 7 and 8 we present dependence of the $[\mathrm{X} / \mathrm{Fe}]$ abundance ratios on the stellar metallicity. The comparison is done for the large primary sample including stars with $\log g<$ $4.1 \mathrm{~cm} \mathrm{~s}^{-2}$. For comparison, the HARPS sample of FGK dwarf stars from Adibekyan et al. (2012c) is plotted as well. The abundances of atomic Carbon and Oxygen for the HARPS sample stars were derived in Delgado Mena et al. (2010) and Bertran de Lis et al. (2015), respectively ${ }^{18}$. Figure 7 shows that the two samples generally follow the same trend set by the Galactic chemical evolution. However, some discrepancies are apparent and worth mentioning. It seems that at metallicities above solar value $\mathrm{Al}$ and especially $\mathrm{Si}$ abundances relative to iron are higher for the current sample when compared to the HARPS sample stars. For the same metallicities, Ca and TiII seem to be underabundant when compared to their HARPS counterparts. Most probably this is because many of the stars of the first sample are giant, evolved stars. Figure 8 shows that indeed, most of the Al- and Si-enhanced, and Ca- and TilI-poor stars are evolved

18 Note that Suárez-Andrés et al. (2017) also derived Carbon abundance for the HARPS sample stars from the $\mathrm{CH}$ band at $4300 \AA$. 
Table 4. Iron-to-silicate and water mass fraction of planet building blocks.

\begin{tabular}{|c|c|c|}
\hline Star & $f_{\text {iron }}(\%)$ & $\mathrm{wf}(\%)$ \\
\hline HIP13402 & $35.0 \pm 3.1$ & $58.1 \pm 7.1$ \\
\hline HIP16852 & $31.0 \pm 2.0$ & $57.3 \pm 4.6$ \\
\hline HIP22263 & $36.5 \pm 1.3$ & $59.6 \pm 7.7$ \\
\hline HIP23835 & $29.0 \pm 1.2$ & $73.1 \pm 2.6$ \\
\hline HIP43587 ${ }^{(\star)}$ & $31.6 \pm 3.0$ & $46.5 \pm 8.0$ \\
\hline HIP64924 ${ }^{(\star)}$ & $33.3 \pm 1.8$ & $49.3 \pm 8.8$ \\
\hline HIP77257 & $33.0 \pm 1.1$ & $58.5 \pm 2.8$ \\
\hline HIP83541 & $31.5 \pm 2.6$ & $47.4 \pm 6.5$ \\
\hline HIP95447 & $35.3 \pm 2.8$ & $48.7 \pm 5.5$ \\
\hline HIP98066 & $34.7 \pm 1.6$ & $51.9 \pm 5.1$ \\
\hline HIP98767 ${ }^{(\star)}$ & $31.2 \pm 2.1$ & $49.4 \pm 4.5$ \\
\hline HIP101345 & $32.9 \pm 1.0$ & $59.3 \pm 3.7$ \\
\hline HIP101916 & $33.5 \pm 1.9$ & $56.2 \pm 4.0$ \\
\hline HIP113357 ${ }^{\star \star)}$ & $34.8 \pm 2.0$ & $41.3 \pm 4.7$ \\
\hline HIP37606 & $31.9 \pm 1.4$ & $57.8 \pm 4.2$ \\
\hline HIP107649 & $34.8 \pm 1.9$ & $50.8 \pm 6.5$ \\
\hline HIP86796 ${ }^{(\star)}$ & $33.2 \pm 2.0$ & $55.1 \pm 4.0$ \\
\hline HIP114699 ${ }^{(\star)}$ & $30.2 \pm 1.1$ & $66.1 \pm 3.6$ \\
\hline HIP29271 & $33.4 \pm 1.8$ & $61.4 \pm 5.6$ \\
\hline HIP1599 & $32.2 \pm 1.4$ & $61.7 \pm 6.1$ \\
\hline HIP8102 ${ }^{(\star)}$ & $24.0 \pm 1.8$ & $66.6 \pm 6.5$ \\
\hline HIP10138 ${ }^{(\star)}$ & $27.6 \pm 1.7$ & $64.0 \pm 6.0$ \\
\hline HIP15330 & $32.0 \pm 1.9$ & $60.1 \pm 9.1$ \\
\hline HIP15371 & $32.4 \pm 1.6$ & $70.8 \pm 5.6$ \\
\hline HIP38908 & $24.6 \pm 1.3$ & $73.2 \pm 3.4$ \\
\hline HIP40693 ${ }^{(\star)}$ & $32.6 \pm 1.6$ & $53.8 \pm 8.1$ \\
\hline HIP56452 & $29.7 \pm 2.0$ & $66.0 \pm 6.9$ \\
\hline HIP57443 & $28.6 \pm 1.6$ & $65.8 \pm 6.6$ \\
\hline HIP81300 & $34.1 \pm 2.3$ & $51.2 \pm 9.2$ \\
\hline HIP110109 & $33.0 \pm 1.2$ & $58.7 \pm 7.4$ \\
\hline
\end{tabular}

Notes. ${ }^{\star}$ Host confirmed exoplanet.

stars with $\log g<3.5$ dex. Several studies observed such differences in chemical abundances between main-sequence dwarfs and evolved stars (e.g., Friel et al. 2003; Villanova et al. 2009; Adibekyan et al. 2015). These differences might have astrophysical origins (e.g., Tautvaišienè et al. 2005, as in the case of Sodium), they might be related to the spectroscopic analysis methods and use line-lists (e.g., Santos et al. 2009) and/or non-LTE effects, which are stronger for evolved stars (e.g., Bergemann et al. 2013). Some of the elements also show a dependence on effective temperature that has already been noticed and discussed in the literature (e.g. Gilli et al. 2006; Lai et al. 2008; Adibekyan et al. 2012c). These trends may be related to the stronger line blendings at low temperatures, deviations from excitation and/or ionization equilibrium, or an incorrect T- $\tau$ relationship in the adopted model atmospheres (see Adibekyan et al. 2012c, for more details and references)

Figure 7 shows that the most iron-poor stars are enhanced in $\alpha$ elements and probably belong to the Galactic thick disk (e.g., Adibekyan et al. 2013; Recio-Blanco et al. 2014). This is very interesting to note since it was found that planets orbiting iron-poor stars prefer the $\alpha$-enhanced ones (e.g., Haywood 2009; Adibekyan et al. 2012a,b). Enhancement of the $\alpha$ elements, such as $\mathrm{Mg}$ and $\mathrm{Si}$, relative to iron can play a very important role not only for the formation of planets, but also on the composition of the formed planets (Santos et al. 2017).
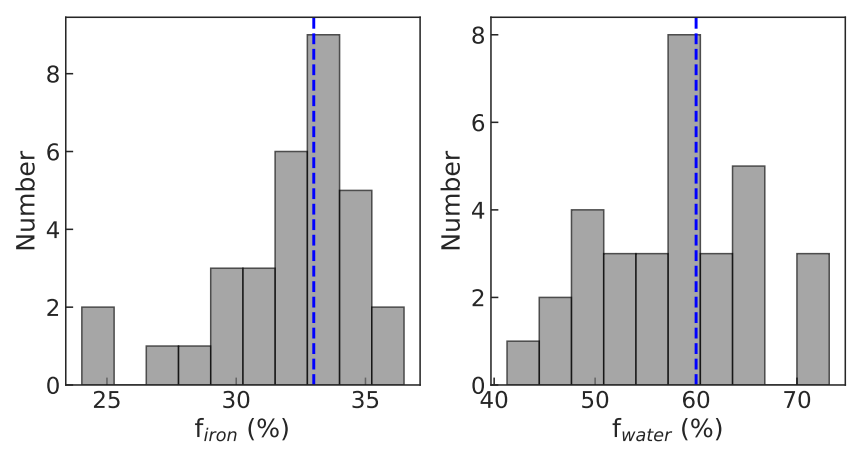

Fig. 9. Expected iron and water mass fraction distributions of planetary building grains around sample stars. The values of these quantities derived for the solar system with our model are shown with dashed blue line.

For the M dwarfs for which spectra were available, we used a modified version of the method and software developed by Neves et al. (2014) to $T_{\text {eff }}$ and $[\mathrm{Fe} / \mathrm{H}]^{19}$. The $T_{\text {eff }}$ scale was updated and its initial values were calculated as the average of the $(V-J),(V-H)$, and $(V-K)$ calibrations taken from Boyajian et al. (2012). The corresponding errors were reported using Tables 5 and 6 in Neves et al. (2014). The values are presented in Tables B.2-B.4. We presented $\log g$ using Eq. (3) and $T_{\text {eff }}$ using color calibrations where the sufficient spectra were not available and the targets were already discarded by the other criteria from the final list. The error bar for these targets were not calculated.

Composition of planetary building blocks. For a sample of 32 solar-type stars we derived abundances of the main rockforming refractory $(\mathrm{Mg}, \mathrm{Si}$, and $\mathrm{Fe})$ and volatile $(\mathrm{O}, \mathrm{C})$ elements. We used the model described in Santos et al. (2017) to compute the expected iron-to-silicate mass fraction $\left(f_{\text {iron }}\right)$ and the water mass fraction (wf) in the planetary building blocks. The derived values of these quantities are presented in Table 4 and their distributions are shown in Fig. 9. The distribution of $f_{\text {iron }}$ and wf are very similar to the distribution of the same quantities derived by Santos et al. (2017) for the Galactic thin disk stars in the solar neighborhood.

\section{Stellar rotational period estimation for $\mathbf{M}$ dwarfs}

The rotation period of $M$ dwarfs can be of the same order of magnitude as the period of a planet in the HZ. The presence of active regions on $\mathrm{M}$ dwarfs can thus produce an RV signal that can mimic the signal produced by an Earth-mass exoplanet inside the HZ or at any harmonic of the rotation period (e.g., Boisse et al. 2009).

We derived the expected rotational period of $M$ dwarfs using activity-rotation relationships as a first estimation. Several studies show a significant correlation between the activity and rotation for M dwarfs (e.g., Suárez Mascareño et al. 2018b, 2016; Astudillo-Defru et al. 2017). We used Eq. (1) and Table 1 from Suárez Mascareño et al. (2015) for the rotational period determination from the $\log \left(R_{\mathrm{HK}}^{\prime}\right)$. In Table 5 , we list the rotational period estimated in this way, as well as the orbital period corresponding to the inner boundary of the $\mathrm{HZ}$ for our sample of $M$ dwarfs. We derived the error using the standard error propagation of the input parameters' errors. For low-activity stars $\left(\log \left(R_{\mathrm{HK}}^{\prime}\right)<-4.80\right)$, the rotation periods shows a large dispersion, specially for the early $M$ spectral types. Therefore, in order

19 http://WWW . astro.up.pt/resources/mcal/Site/MCAL .html 
Table 5. Sample of M dwarf in final list with rotational periods and $\mathrm{HZ}$ parameters.

\begin{tabular}{|c|c|c|c|c|}
\hline Star name & $P_{\text {rot }}$ (day) & Stellar mass $\left(M_{\odot}\right)$ and reference & $P_{\text {inner }}$ (day) & $\mathrm{HZ}(\mathrm{AU})$ \\
\hline HIP439 & $79 \pm 6$ & $0.39 \pm 0.03[1]$ & $25 \pm 1$ & {$[0.12,0.32]$} \\
\hline HIP1242 & $192 \pm 8$ & $0.14[2]$ & 12 & {$[0.05,0.14]$} \\
\hline HIP22627 & $64 \pm 6$ & $0.37 \pm 0.06[3]$ & $20 \pm 2$ & {$[0.1,0.28]$} \\
\hline HIP23708 & $33 \pm 5$ & $0.59 \pm 0.02[4]$ & $55 \pm 1$ & {$[0.24,0.61]$} \\
\hline HIP29295 & $29 \pm 5$ & $0.58 \pm 0.06[1]$ & $41 \pm 2$ & {$[0.19,0.5]$} \\
\hline HIP40239 & $161 \pm 8$ & $0.61[2]$ & 49 & {$[0.22,0.57]$} \\
\hline HIP42748 & $81 \pm 6$ & $0.45 \pm 0.05[4]$ & $60 \pm 3$ & {$[0.23,0.59]$} \\
\hline HIP45908 & $31 \pm 5$ & $0.55 \pm 0.03[1]$ & $38 \pm 1$ & {$[0.18,0.46]$} \\
\hline HIP51317 & $46 \pm 5$ & $0.44 \pm 0.03[1]$ & $26 \pm 1$ & {$[0.13,0.34]$} \\
\hline HIP53020 & $88 \pm 6$ & $0.26 \pm 0.02[1]$ & $14 \pm 1$ & {$[0.07,0.2]$} \\
\hline HIP62452 & $104 \pm 7$ & $0.32 \pm 0.02[1]$ & $16 \pm 1$ & {$[0.09,0.23]$} \\
\hline HIP65859 & $30 \pm 5$ & $0.53 \pm 0.03[1]$ & $34 \pm 1$ & {$[0.17,0.43]$} \\
\hline HIP67164 & $80 \pm 6$ & $0.42 \pm 0.06[5]$ & $14 \pm 1$ & {$[0.09,0.23]$} \\
\hline HIP71253 & $85 \pm 6$ & $0.28 \pm 0.02[1]$ & $15 \pm 1$ & {$[0.08,0.21]$} \\
\hline HIP85523 & $38 \pm 5$ & $0.35 \pm 0.03[1]$ & $21 \pm 1$ & {$[0.1,0.27]$} \\
\hline HIP86214 & $35 \pm 5$ & $0.27 \pm 0.02[1]$ & $15 \pm 1$ & {$[0.08,0.21]$} \\
\hline HIP86287 & $73 \pm 6$ & $0.45 \pm 0.03[1]$ & $28 \pm 1$ & {$[0.14,0.36]$} \\
\hline HIP87937 & $73 \pm 6$ & $0.16 \pm 0.01[1]$ & $9 \pm 1$ & {$[0.04,0.12]$} \\
\hline HIP88574 & $46 \pm 5$ & $0.48 \pm 0.03[1]$ & $30 \pm 1$ & {$[0.15,0.38]$} \\
\hline HIP92403 & $30 \pm 5$ & $0.17 \pm 0.01[1]$ & $11 \pm 1$ & {$[0.05,0.14]$} \\
\hline HIP93069 & $49 \pm 5$ & $0.53[2]$ & 59 & {$[0.24,0.62]$} \\
\hline HIP93101 & $28 \pm 4$ & $0.43 \pm 0.05[4]$ & $51 \pm 3$ & {$[0.2,0.52]$} \\
\hline HIP99701 & $26 \pm 4$ & $0.59[2]$ & 42 & {$[0.2,0.51]$} \\
\hline HIP106440 & $52 \pm 5$ & $0.45 \pm 0.03[1]$ & $28 \pm 1$ & {$[0.14,0.35]$} \\
\hline HIP113020 & $112 \pm 7$ & $0.34 \pm 0.02[1]$ & $18 \pm 1$ & {$[0.09,0.25]$} \\
\hline HIP115332 & $44 \pm 5$ & $0.4 \pm 0.02[1]$ & $20 \pm 1$ & {$[0.1,0.28]$} \\
\hline HIP67155 & $38 \pm 5$ & $0.5 \pm 0.03[1]$ & $29 \pm 1$ & {$[0.14,0.37]$} \\
\hline
\end{tabular}

References. [1]: Neves et al. (2013), [2]: Gaidos et al. (2014), [3]: Mann et al. (2015), [4]: Mints \& Hekker (2017), [5]: Gaidos \& Mann (2014).

to take into account the actual dispersion of the data points in Fig. 12 from Suárez Mascareño et al. (2018b), we considered an uncertainty in the rotation period inflated by a factor of three.

We are aware that the rotation periods obtained in this way are a first-order estimation. Although the statistical strength of the relationship for the purpose of this study is sufficient enough, we did not exclude any target from the final list based on this criterion. There are some stars for which extreme caution is necessary because the rotational period of the stars are very close to the HZ inner edge's orbital period. For these targets distinguishing the planetary signal from RV variations of stars due to its rotation will be quite challenging. The measurements can be improved in the future studies using either photometric or spectroscopic methods.

\section{Summary and conclusion}

We present a detailed spectroscopical characterization of a sample of bright stars that are suitable targets for a precise RV planet search program focused on Earth-mass planets in their hosts habitable zone using ESPRESSO. To build this sample, we used new and archival high-resolution spectroscopic data. We screened a large sample of $\mathrm{G}, \mathrm{K}$, and $\mathrm{M}$ stars for visibility, spectroscopic contamination, chromosphere activity using $\log \left(R_{\mathrm{HK}}^{\prime}\right)$, projected rotational velocity using $v \sin i$, stellar parameters, and chemical abundances. After considering previous criteria, for the known host planetary systems we investigated the availability of a free, stable orbit for an Earth-mass planet inside the HZ.
Twenty-seven stars were discarded according to the signature of stellar companions in CCF criteria. Forty-three stars with high activity $\log \left(R_{\mathrm{HK}}^{\prime}\right)>-4.80$ were excluded from the list due to the expected large activity-induced RV variations. We identified twenty-three stars with $v \sin i>5.0 \mathrm{~km} \mathrm{~s}^{-1}$ and excluded them from the final list. The list of the 45 best targets is presented in Table 6. For the known host planetary systems which were described in Sect. 6, nineteen best candidates are presented in Table 3. Five G, fifteen $\mathrm{K}$, and twenty-seven $\mathrm{M}$ spectral type stars have $V$ between 3.49 and 11.94. Figures 10 and 11 present the distribution of $[\mathrm{Fe} / \mathrm{H}]$ for forty stars with available chemical abundance values.

When performing our study we realized that many bright, main-sequence GKM stars did not have sufficiently high $\mathrm{S} / \mathrm{N}$ spectra in the ESO archive to allow spectroscopic analysis. The spectra obtained by us were made available immediately, and the reduced spectra, made available now, can be of interest to a wide range of studies. All the spectra are available for download from the ESO archive and also from our Porto data server for the case of HARPS-N observations ${ }^{20}$.

Concerning the final target list, applying the criteria on spectroscopic contamination, stellar activity, and chromospheric activity levels, and discarding evolved stars by a direct or indirect assessment of the $\log g$, led us to discard a large fraction of the stars. At the end of the selection steps, we retained only $\sim 45$ of the $\sim 249$ stars originally surveyed spectroscopically. This selection, which was devised to be independent of spectral type in any

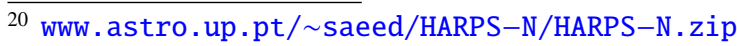


Table 6. Forty-five best final targets for RV observation.

\begin{tabular}{|c|c|c|c|c|c|c|c|}
\hline Name & RA (hh:mm:ss) & $\operatorname{Dec}\left({ }^{\circ}:^{\prime}:^{\prime \prime}\right)$ & $v \sin i\left(\mathrm{~km} \mathrm{~s}^{-1}\right)$ & $\log \left(R_{\mathrm{HK}}^{\prime}\right)$ & $\log I_{\mathrm{H} \alpha_{\text {index }}}$ & V & Sp type \\
\hline HIP86796 & 174408.7 & -515000.9 & $2.95 \pm 0.90$ & $-5.15 \pm 0.01$ & $-1.37 \pm 0.07$ & 5.12 & G5 \\
\hline HIP83541 & 170427.8 & -283455.3 & $<2.0$ & $-5.02 \pm 0.01$ & $-1.43 \pm 0.01$ & 6.59 & G8 \\
\hline HIP57443 & 114632.2 & -403004.8 & $<2.0$ & $-4.88 \pm 0.01$ & $-1.49 \pm 0.06$ & 4.89 & G8 \\
\hline HIP64924 & 131825.0 & -181831.0 & $<2.0$ & $-4.91 \pm 0.01$ & $-1.45 \pm 0.01$ & 4.74 & G8 \\
\hline HIP40693 & 081823.8 & -123747.2 & $<2.0$ & $-4.94 \pm 0.01$ & $-1.46 \pm 0.09$ & 5.95 & G8 \\
\hline HIP41926 & 083252.2 & -31 3009.7 & - & $-4.91 \pm 0.01$ & $-1.43 \pm 0.10$ & 6.38 & K0 \\
\hline HIP15510 & 031953.2 & -430417.6 & - & $-5.00 \pm 0.01$ & $-1.45 \pm 0.01$ & 4.26 & K0 \\
\hline HIP8102 & 014405.1 & -155622.4 & - & $-4.90 \pm 0.01$ & $-1.44 \pm 0.07$ & 3.49 & K0 \\
\hline HIP19849 & 041517.6 & -073840.4 & $<2.0$ & $-4.92 \pm 0.01$ & $-1.41 \pm 0.01$ & 4.43 & K1 \\
\hline HIP99825 & 201516.6 & -270157.1 & $<2.0$ & $-4.96 \pm 0.01$ & $-1.41 \pm 0.01$ & 5.73 & $\mathrm{~K} 1$ \\
\hline HIP56452 & 113430.0 & -325000.0 & - & $-4.83 \pm 0.04$ & $-1.44 \pm 0.10$ & 5.96 & K1 \\
\hline HIP3765 & 004822.5 & +051700.2 & - & $-4.93 \pm 0.01$ & $-1.41 \pm 0.01$ & 5.74 & $\mathrm{~K} 2$ \\
\hline HIP99461 & 201111.6 & -360550.6 & $<2.0$ & 1.50 & $-1.41 \pm 0.02$ & 5.32 & $\mathrm{~K} 2$ \\
\hline HIP12114 & 023603.8 & +065300.1 & $2.00 \pm 0.27$ & $-4.96 \pm 0.01$ & $-1.38 \pm 0.04$ & 5.79 & $\mathrm{~K} 2$ \\
\hline HIP23311 & 050048.7 & -054503.5 & $<2.0$ & $-5.00 \pm 0.01$ & $-1.39 \pm 0.01$ & 6.22 & K3 \\
\hline HIP16711 & 033500.5 & -482511.6 & $<2.0$ & $-5.09 \pm 0.01$ & $-1.52 \pm 0.01$ & 8.57 & K4 \\
\hline HIP108870 & 220317.4 & -564647.3 & $<2.0$ & $-4.80 \pm 0.01$ & $-1.34 \pm 0.07$ & 4.69 & K4 \\
\hline HIP85647 & 173011.2 & -513813.0 & $2.64 \pm 0.36$ & $-5.12 \pm 0.02$ & $-1.53 \pm 0.04$ & 9.58 & K7 \\
\hline HIP40239 & 081308.5 & -135501.0 & $4.89 \pm 0.23$ & $-5.65 \pm 0.25$ & $-1.39 \pm 0.07$ & 9.38 & M0 \\
\hline HIP29295 & 061034.6 & -215152.4 & $2.61 \pm 0.41$ & $-4.90 \pm 0.01$ & $-1.51 \pm 0.02$ & 8.15 & M1 \\
\hline HIP439 & 000524.2 & -372125.3 & $<2.0$ & $-5.47 \pm 0.01$ & $-1.36 \pm 0.02$ & 8.56 & M1 \\
\hline HIP42748 & 084244.5 & +093324.4 & $2.67 \pm 0.72$ & $-5.48 \pm 0.10$ & $-1.39 \pm 0.02$ & 9.62 & M1 \\
\hline HIP45908 & 092137.7 & -601655.1 & $2.32 \pm 0.65$ & $-4.94 \pm 0.01$ & $-1.46 \pm 0.02$ & 9.49 & M1 \\
\hline HIP93101 & 185800.1 & +055429.8 & $2.44 \pm 0.65$ & $-4.86 \pm 0.01$ & $-1.46 \pm 0.02$ & 9.22 & M1 \\
\hline HIP93069 & 185730.6 & -555930.6 & $<2.0$ & $-5.19 \pm 0.01$ & - & 8.86 & M1 \\
\hline HIP23708 & 050547.4 & -573313.8 & $<2.0$ & $-4.96 \pm 0.01$ & $-1.42 \pm 0.05$ & 9.11 & M2 \\
\hline HIP51317 & 102855.6 & +00 5028.0 & $2.28 \pm 0.20$ & $-5.16 \pm 0.02$ & $-1.41 \pm 0.01$ & 9.65 & M2 \\
\hline HIP86287 & 173753.3 & +183529.7 & $2.63 \pm 0.63$ & $-5.43 \pm 0.01$ & $-1.50 \pm 0.02$ & 9.62 & $\mathrm{M} 2$ \\
\hline HIP88574 & 180507.6 & -30152.6 & $2.47 \pm 0$ & $-5.16 \pm 0.01$ & $-1.51 \pm 0.02$ & 9.37 & M2 \\
\hline HIP106440 & 213334.0 & -490032.0 & $2.09 \pm 0.26$ & $-5.23 \pm 0.01$ & $-1.48 \pm 0.02$ & 8.66 & $\mathrm{M} 2$ \\
\hline HIP65859 & 132959.7 & +102238.3 & $2.55 \pm 0.20$ & $-4.91 \pm 0.01$ & $-1.50 \pm 0.02$ & 9.05 & M2 \\
\hline HIP99701 & 201353.3 & -450950.4 & $<2.0$ & $-4.84 \pm 0.06$ & $-1.46 \pm 0.02$ & 7.97 & M2 \\
\hline HIP67155 & 134542.7 & +145342.2 & - & $-5.04 \pm 0.01$ & $-1.44 \pm 0.01$ & 8.46 & M2 \\
\hline HIP85523 & 172839.9 & -465342.2 & $2.59 \pm 0.34$ & $-5.05 \pm 0.01$ & $-1.22 \pm 0.03$ & 9.38 & M3 \\
\hline HIP71253 & 143416.8 & -123110.7 & $2.83 \pm 0.51$ & $-5.51 \pm 0.01$ & $-1.43 \pm 0.04$ & 11.32 & M4 \\
\hline HIP1242 & 001528.1 & -160801.4 & $4.30 \pm 0.27$ & $-5.68 \pm 0.21$ & $-1.38 \pm 0.04$ & 11.49 & M6 \\
\hline HIP53020 & 105052.1 & +064829.7 & $4.92 \pm 0.22$ & $-5.53 \pm 0.10$ & $-1.43 \pm 0.10$ & 11.64 & M6 \\
\hline HIP87937 & 175748.5 & +04 4131.0 & - & $-5.42 \pm 0.01$ & $-1.32 \pm 0.07$ & 9.54 & M6 \\
\hline HIP92403 & 184949.3 & -235010.3 & $5.20 \pm 0.91$ & $-4.91 \pm 0.02$ & $-0.98 \pm 0.01$ & 10.37 & M6 \\
\hline HIP115332 & 232137.5 & +17 1726.1 & $4.94 \pm 0.23$ & $-5.13 \pm 0.20$ & $-1.37 \pm 0.05$ & 11.70 & M6 \\
\hline HIP22627 & 045205.7 & +062835.7 & $3.82 \pm 0.80$ & $-5.35 \pm 0.04$ & $-1.39 \pm 0.03$ & 11.94 & M6 \\
\hline HIP62452 & 124756.7 & +094505.2 & $4.30 \pm 0.24$ & $-5.63 \pm 0.10$ & $-1.41 \pm 0.01$ & 11.39 & M6 \\
\hline HIP113020 & 225316.7 & -141549.0 & $2.75 \pm 1.03$ & $-5.67 \pm 0.02$ & $-1.47 \pm 0.01$ & 10.16 & M6 \\
\hline HIP67164 & 134550.7 & -175805.3 & $2.52 \pm 0.25$ & $-5.48 \pm 0.04$ & - & 11.81 & M6 \\
\hline HIP86214 & 173703.7 & -441908.7 & $3.42 \pm 0.79$ & $-4.99 \pm 0.01$ & $-1.44 \pm 0.05$ & 10.94 & M7 \\
\hline
\end{tabular}

other aspect than photon noise, let to a catalog that is composed of roughly of $60 \%$ of $\mathrm{M}$ dwarfs. This is the first study that leads to the conclusion that, even when trying to be as independent as possible of spectral-type biases, when focusing on objective criteria such as photon noise and signal detectability from first principle, M dwarfs emerge as optimal RV targets, even at optical wavelengths.

Most of the stars (27) in the final list are of M spectral type. $\mathrm{M}$ dwarfs are good candidates not only for gravitational RV detection because of their relativity low mass, but also for having a closer HZ. Hypothetical planets around M dwarfs would have relatively shorter orbital periods, making it is easier to cover their orbital period. However M dwarfs with rotational periods close to the orbital period of an Earth-class planet in their $\mathrm{HZ}$ will be considered as of lower priority for observations. The sample of M dwarfs in our final list consists mostly of early-type and late-type spectral subtypes with very few in the intermediate subtypes. M dwarfs are not a homogeneous class of stars and there are many intermediate $\mathrm{M}$ dwarfs in this magnitude regime (see Fig. 5 in Figueira et al. 2016), so this came probably from statistical fluctuations arising from small number statistics. It should be mentioned that the temperature of the $\mathrm{M}$ dwarfs scatter over a few hundreds of kelvins in the different catalogs therefore we cannot compare the parameters in the subspectral $\mathrm{M}$ dwarfs. The effective temperature determination of $\mathrm{M}$ dwarfs is affected by an uncertainty at the level of $100 \mathrm{~K}$. As such, a detailed spectral 


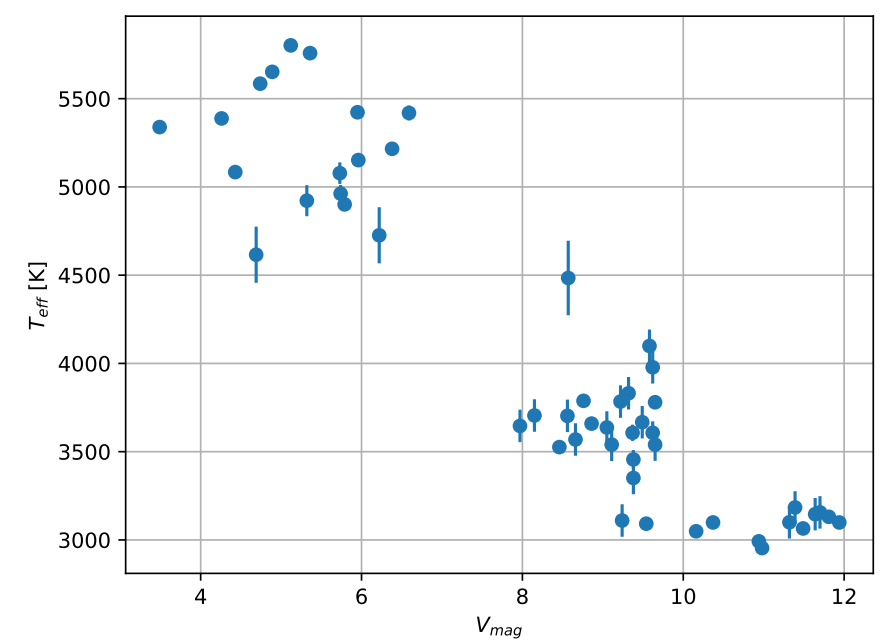

Fig. 10. Visual magnitude vs $T_{\text {eff }}(\mathrm{K})$ for final selected sample of stars.

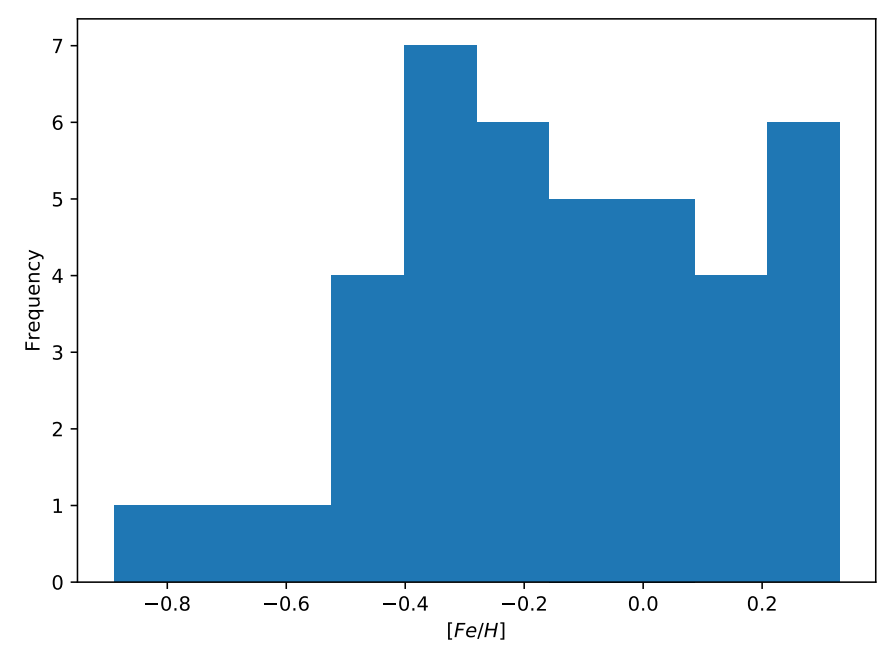

Fig. 11. $[\mathrm{Fe} / \mathrm{H}]$ distribution for final selected sample of stars where metallically values are available.

type assignment based on effective temperatures is impossible, and we warn the reader against an over-interpretation of the data.

We considered stars for which the photon noise allowed the detection of an Earth-class planet inside the HZ. In the absence of sources of error other than the photon noise, an Earth-class planet is detectable in almost all of the candidates with orbital periods of $\approx 6$ to $\approx 223$ days by assuming the expected RV precision of $10 \mathrm{~cm} \mathrm{~s}^{-1}$. Eighty percent of the ESPRESSO GTO is dedicated to exoplanet searches and one-third of this time is allocated to the blind RV survey (65 nights). By considering $9 \mathrm{~h}$ as an average of night duration, an average of 100 data points in order to cover the orbital periods and 15-min exposure time, we can intensively observe around 23 targets in this survey.

The composition of the planetary building block analysis suggests that the potential terrestrial planets orbiting the majority of these stars will have an iron core-size similar to that of the Earth, but will have a lower fraction of water content. It is also interesting to note that (based only on the chemistry of the host stars) terrestrial planets with very different compositions (e.g., core size) and water content when compared to the solar system, are also expected to be detected around some of the targets. Here we should note that the $\mathrm{f}_{\text {iron }}$ and wf derived with this model might not necessarily be exactly the same as the water content and core size of planets around the target stars (see Santos et al. 2017). These quantities are only indicative of the content of water in the protoplanetary disk and the iron-to-silicate ratio that might be translated into a core-to-mantle ratio.

Presently there is no clear indication if the presence of close-in planets (that have been detected e.g., with HARPS) is indicative of the presence of low-mass planets in the habitable zone. On one side, we know from Kepler data that some planetary systems are compact and dynamically full (Fabrycky et al. 2014). If this is a general feature, then the presence of close-in planets could be a positive indication of the presence of planets further out (whatever their mass). Since a giant planet in the HZ of such systems would already have been detected with HARPS, planets in the HZ should be rather small. On the other side, we know from the only confirmed Earth-like planet in the HZ (the Earth itself), that the absence of close-in planets is compatible with the presence of a low-mass planet in the HZ. Finally there are indications, from planet formation models, that the absence of close-in planets could be positively correlated with the presence of low-mass planets in the HZ (Alibert et al., in prep.). Since there is no clear consensus on the correlation between the presence of already detected planets and the probability of having a planet in the HZ, we decided to include both types of stars (with or without already known planets) in our target list.

All derived parameters for the whole sample are presented in Table B.1 for our new observation and archives respectively. We derived the stellar parameters and chemical abundances for this sample of stars (Tables B.2-B.4) where it was possible.

Acknowledgements. This work was supported by FCT/MCTES through national funds and by FEDER - Fundo Europeu de Desenvolvimento Regional through COMPETE2020 - Programa Operacional Competitividade e Internacionalização by these grants: UID/FIS/04434/2019; PTDC/FIS-AST/32113/2017 \& POCI-01-0145-FEDER-032113; PTDC/FIS-AST/28953/2017 \& POCI-01-0145FEDER-028953. This work was supported by FEDER - Fundo Europeu de Desenvolvimento Regional through COMPETE2020 - Programa Operacional Competitividade e Internacionalização (POCI-01-0145-FEDER-028987). S.H. acknowledge support by the fellowships PD/BD/128119/2016 funded by FCT (Portugal). V.A., S.G.S. and E.D.M. acknowledge support from FCT through Investigador FCT contracts nrs. IF/00650/2015/CP1273/CT0001; IF/00028/2014/CP1215/CT0002; IF/00849/2015/CP1273/CT0003. J.H.C.M. is supported in the form of work contract (DL 57/2016/CP1364/CT0007) funded by national funds through FCT. M.O. acknowledges research funding from the Deutsche Forschungsgemeinschft (DFG, German Research Foundation) - OS 508/1-1. A.F.L and G.M. acknowledges the support by INAF/Frontiera through the "Progetti Premiali" funding scheme of the Italian Ministry of Education, University, and Research. J.I.G.H and R.R. acknowledge financial support from the Spanish Ministry project MINECO AYA2017-86389-P. J.I.G.H also acknowledges financial support from the Spanish MINECO under the 2013 Ramón y Cajal program MINECO RYC-2013-14875. J.H.C.M. is supported in the form of a work contract funded by national funds through Fundação para a Ciência e Tecnologia (FCT). B.R.-A. acknowledges the support from CONICYT PAI/Concurso Nacional Inserción en la Academia, Convocatoria 2015 79150050. This work results within the collaboration of the COST Action TD 1308. This research has made use of the SIMBAD database, operated at CDS, Strasbourg, France Wenger et al. (2000) and has made use of the VizieR catalogue access tool, CDS, Strasbourg, France, the IRAF facility and the VALD3 database. This research has made use of the NASA Exoplanet Archive, which is operated by the California Institute of Technology, under contract with the National Aeronautics and Space Administration under the Exoplanet Exploration Program. This work has made use of data from the European Space Agency (ESA) mission Gaia (https://www. cosmos.esa.int/gaia), processed by the Gaia Data Processing and Analysis Consortium (DPAC, https://www. cosmos.esa.int/web/gaia/dpac/consortium). Funding for the DPAC has been provided by national institutions, in particular the institutions participating in the Gaia Multilateral Agreement. We thank the referee for the critical analysis and constructive comments. 


\section{References}

Adibekyan, V., Figueira, P., \& Santos, N. C. 2016, Orig. Life Evol. Biospheres, 46,351

Adibekyan, V. Z., Benamati, L., Santos, N. C., et al. 2015, MNRAS, 450, 1900

Adibekyan, V. Z., Delgado Mena, E., Sousa, S. G., et al. 2012a, A\&A, 547, A36

Adibekyan, V. Z., Figueira, P., Santos, N. C., et al. 2013, A\&A, 554, A44

Adibekyan, V. Z., Santos, N. C., Sousa, S. G., et al. 2012b, A\&A, 543, A89

Adibekyan, V. Z., Sousa, S. G., Santos, N. C., et al. 2012c, A\&A, 545, A32

Andreasen, D., Sousa, S., Tsantaki, M., et al. 2017, A\&A, 600, A69

Anglada-Escudé, G., Amado, P. J., Barnes, J., et al. 2016, Nature, 536, 437

Astudillo-Defru, N., Delfosse, X., Bonfils, X., et al. 2017, A\&A, 600, A13

Baranne, A., Queloz, D., Mayor, M., et al. 1996, A\&AS, 119, 373

Bergemann, M., Kudritzki, R.-P., Würl, M., et al. 2013, ApJ, 764, 115

Bertran de Lis, S., Delgado Mena, E., Adibekyan, V. Z., Santos, N. C., \& Sousa, S. G. 2015, A\&A, 576, A89

Boisse, I., Moutou, C., Vidal-Madjar, A., et al. 2009, A\&A, 495, 959

Bonfils, X., Mayor, M., Delfosse, X., et al. 2007, A\&A, 474, 293

Bouchy, F., Pepe, F., \& Queloz, D. 2001, A\&A, 374, 733

Bouchy, F., Ségransan, D., Díaz, R. F., et al. 2016, A\&A, 585, A46

Boyajian, T. S., von Braun, K., van Belle, G., et al. 2012, ApJ, 757, 112

Brown, A., Vallenari, A., Prusti, T., et al. 2018, A\&A, 616, A1

Browning, M. K., Basri, G., Marcy, G. W., West, A. A., \& Zhang, J. 2010, AJ 139,504

Carroll, B. W., \& Ostlie, D. A. 2017, An Introduction to Modern Astrophysics (Cambridge: Cambridge University Press)

Casagrande, L., Flynn, C., \& Bessell, M. 2008, MNRAS, 389, 585

Casagrande, L., Ramírez, I., Melendez, J., Bessell, M., \& Asplund, M. 2010, A\&A, 512, A54

Cincunegui, C., Díaz, R. F., \& Mauas, P. J. 2007, A\&A, 469, 309

Cosentino, R., Lovis, C., Pepe, F., et al. 2012, in Ground-based and Airborne Instrumentation for Astronomy IV, Proc. SPIE, 8446, $84461 \mathrm{~V}$

Cunha, D., Figueira, P., Santos, N., Lovis, C., \& Boué, G. 2013, A\&A, 550, A75

Cutri, R., Skrutskie, M., Van Dyk, S., et al. 2003, VizieR Online Data Catalog: II $/ 246$

da Silva, L., Girardi, L., Pasquini, L., et al. 2006, A\&A, 458, 609

Dekker, H., D’Odorico, S., Kaufer, A., Delabre, B., \& Kotzlowski, H. 2000, in Optical and IR Telescope Instrumentation and Detectors, eds. M. Iye \& A. F. Moorwood, Proc. SPIE, 4008, 534

Delgado Mena, E., Israelian, G., González Hernández, J. I., et al. 2010, ApJ, 725, 2349

Díaz, R., Ségransan, D., Udry, S., et al. 2016, A\&A, 585, A134

Dumusque, X., Boisse, I., \& Santos, N. 2014, ApJ, 796, 132

Dumusque, X., Udry, S., Lovis, C., Santos, N. C., \& Monteiro, M. 2011, A\&A 525, A 140

Duncan, D. K., Vaughan, A. H., Wilson, O. C., et al. 1991, ApJS, 76, 383

Fabrycky, D. C., Lissauer, J. J., Ragozzine, D., et al. 2014, ApJ, 790, 146

Figueira, P. 2018, in Asteroseismology and Exoplanets: Listening to the Stars and Searching for New Worlds (Berlin: Springer), 181

Figueira, P., Adibekyan, V. Z., Oshagh, M., et al. 2016, A\&A, 586, A101

Fischer, D. A., \& Valenti, J. 2005, ApJ, 622, 1102

Friel, E. D., Jacobson, H. R., Barrett, E., et al. 2003, AJ, 126, 2372

Gaidos, E., Mann, A., Lépine, S., et al. 2014, MNRAS, 443, 2561

Gaidos, E., \& Mann, A. W. 2014, ApJ, 791, 54

Gilli, G., Israelian, G., Ecuvillon, A., Santos, N. C., \& Mayor, M. 2006, A\&A, 449, 723

Gladman, B. 1993, Icarus, 106, 247

Gomes da Silva, J., Santos, N., Bonfils, X., et al. 2011, A\&A, 534, A30

Gomes da Silva, J., Santos, N. C., Boisse, I., Dumusque, X., \& Lovis, C. 2014, A\&A, 566, A66

González Hernández, J. I., Pepe, F., Molaro, P., \& Santos, N. 2018, Handbook of Exoplanets (Berlin: Springer International Publishing AG)

Hatzes, A. c.-a., Guenther, E., Endl, M., et al. 2005, A\&A, 437, 743

Hatzes, A. P. 2013a, Astron. Nachr., 334, 616

Hatzes, A. P. 2013b, ApJ, 770, 133

Haywood, M. 2009, ApJ, 698, L1

Hekker, S., Snellen, I., Aerts, C., et al. 2008, A\&A, 480, 215

Hinkel, N. R., Young, P. A., Pagano, M. D., et al. 2016, ApJS, 226, 4

Jofré, P., Heiter, U., Worley, C. C., et al. 2017, A\&A, 601, A38

Kuerster, M., Endl, M., Rouesnel, F., et al. 2003, A\&A, 403, 1077

Kurucz, R. L. 1993, SYNTHE Spectrum Synthesis Programs and Line Data (Cambridge, MA: Smithsonian Astrophysical Observatory)

Lai, D. K., Bolte, M., Johnson, J. A., et al. 2008, ApJ, 681, 1524
Lépine, S., \& Gaidos, E. 2011, AJ, 142, 138

Lovis, C., \& Mayor, M. 2007, A\&A, 472, 657

Lovis, C., Ségransan, D., Mayor, M., et al. 2011, A\&A, 528, A112

Maldonado, J., Scandariato, G., Stelzer, B., et al. 2017, A\&A, 598, A27

Mann, A. W., Feiden, G. A., Gaidos, E., Boyajian, T., \& von Braun, K. 2015, ApJ, 804, 64

Martins, J. H. C., \& Figueira, P. 2017, UVES data reduction suite, https:// github.com/jorgehumberto/UVES_reduction

Mayor, M., Pepe, F., Queloz, D., et al. 2003, The Messenger, 114, 20

Mayor, M., \& Queloz, D. 1995, Nature, 378, 355

Mayor, M., Udry, S., Naef, D., et al. 2004, A\&A, 415, 391

Meunier, N., Desort, M., \& Lagrange, A.-M. 2010, A\&A, 512, A39

Middelkoop, F. 1982, A\&A, 107, 31

Mints, A., \& Hekker, S. 2017, A\&A, 604, A108

Neal, J., \& Figueira, P. 2019, J. Open Source Softw., 4, 1053

Neves, V., Bonfils, X., Santos, N., et al. 2013, A\&A, 551, A36

Neves, V., Bonfils, X., Santos, N., et al. 2014, A\&A, 568, A121

Noyes, R. W., Hartmann, L. W., Baliunas, S. L., Duncan, D. K., \& Vaughan, A. H. 1984, ApJ, 279, 763

Oshagh, M., Santos, N. C., Figueira, P., et al. 2017, A\&A, 606, A107

Pasquini, L., \& Pallavicini, R. 1991, A\&A, 251, 199

Pepe, F., Ehrenreich, D., \& Meyer, M. R. 2014a, Nature, 513, 358

Pepe, F., Mayor, M., Galland, F., et al. 2002, A\&A, 388, 632

Pepe, F., Molaro, P., Cristiani, S., et al. 2014b, Astron. Nachr., 335, 8

Pepe, F. A., Cristiani, S., Lopez, R. R., et al. 2010, in Ground-based and Airborne Instrumentation for Astronomy III, Int. Soc. for Opt. Photon., 7735, 77350F

Perryman, M. 2011, The Exoplanet Handbook (Cambridge: Cambridge University Press)

Perryman, M. A. C., Lindegren, L., Kovalevsky, J., et al. 1997, A\&A, 323, L49

Pourbaix, D., Tokovinin, A. A., Batten, A. H., et al. 2004, A\&A, 424, 727

Queloz, D., Henry, G., Sivan, J., et al. 2001, A\&A, 379, 279

Recio-Blanco, A., de Laverny, P., Kordopatis, G., et al. 2014, A\&A, 567, A5

Reffert, S., Bergmann, C., Quirrenbach, A., Trifonov, T., \& Künstler, A. 2015 A\&A, 574, A116

Reiners, A., Zechmeister, M., Caballero, J. A., et al. 2018, A\&A, 612, A49

Ricker, G. R., Winn, J. N., Vanderspek, R., et al. 2014, J. Astron. Telesc. Instrum. Syst., 1, 014003

Robertson, P., Mahadevan, S., Endl, M., \& Roy, A. 2014, Science, 345, 440

Santerne, A., Brugger, B., Armstrong, D., et al. 2018, Nat. Astron., 2, 393

Santos, N., Adibekyan, V., Dorn, C., et al. 2017, A\&A, 608, A94

Santos, N., Mortier, A., Faria, J., et al. 2014, A\&A, 566, A35

Santos, N., Sousa, S., Mortier, A., et al. 2013, A\&A, 556, A150

Santos, N. C., Adibekyan, V., Dorn, C., et al. 2017, A\&A, 608, A94

Santos, N. C., Israelian, G., \& Mayor, M. 2004, A\&A, 415, 1153

Santos, N. C., Lovis, C., Pace, G., Melendez, J., \& Naef, D. 2009, A\&A, 493, 309

Santos, N. C., Mayor, M., Naef, D., et al. 2002, A\&A, 392, 215

Selsis, F., Kasting, J., Levrard, B., et al. 2007, A\&A, 476, 1373

Setiawan, J. c.-a., Pasquini, L., Da Silva, L., et al. 2004, A\&A, 421, 241

Sneden, C. A. 1973, PhD Thesis, The University of Texas at Austin, USA

Sousa, S. G. 2014 Determination of Atmospheric Parameters of B-, A-, F- and G-Type Stars, GeoPlanet: Earth and Planetary Sciences, eds. E. Niemczura, B. Smalley, \& W. Pych (Cham: Springer International Publishing), 297

Sousa, S. G., Santos, N. C., Adibekyan, V., Delgado-Mena, E., \& Israelian, G. 2015, A\&A, 577, A67

Stassun, K. G., Oelkers, R. J., Pepper, J., et al. 2018, AJ, 156, 102

Suárez-Andrés, L., Israelian, G., González Hernández, J. I., et al. 2017, A\&A, 599, A96

Suárez Mascareño, A., Rebolo, R., González Hernández, J., \& Esposito, M. 2015, MNRAS, 452, 2745

Suárez Mascareño, A., Rebolo, R., \& González Hernández, J. I. 2016, A\&A, 595, A12

Suárez Mascareño, A., Rebolo, R., González Hernández, J., \& Esposito, M. 2017, MNRAS, 468, 4772

Suárez Mascareño, A., González Hernández, J. I., Rebolo, R., et al. 2018a, A\&A, 612, A41

Suárez Mascareño, A., Rebolo, R., González Hernández, J. I., et al. 2018b, A\&A, 612, A89

Tautvaišienè, G., Edvardsson, B., Puzeras, E., \& Ilyin, I. 2005, A\&A, 431, 933

Torres, G. 2010, AJ, 140, 1158

Vaughan, A. H., \& Preston, G. W. 1980, PASP, 92, 385

Vaughan, A. H., Preston, G. W., \& Wilson, O. C. 1978, PASP, 90, 267

Villanova, S., Carraro, G., \& Saviane, I. 2009, A\&A, 504, 845

Wenger, M., Ochsenbein, F., Egret, D., et al. 2000, A\&AS, 143, 9 


\section{Appendix A: $\boldsymbol{H}_{\alpha}$ index}

On top of the $\log \left(R_{\mathrm{HK}}^{\prime}\right)$ index, we also computed, where it was possible for all our stars, an activity index based on the $H_{\alpha}$ line. The activity characterization of $\mathrm{M}$ dwarfs is made more difficult than for $\mathrm{G}$ and $\mathrm{K}$ counterparts due to the comparatively lower flux in the wavelength region corresponding to the $\mathrm{Ca}$ II $\mathrm{H} \& \mathrm{~K}$. Fortunately, several alternatives exist in the optical domain. It was demonstrated that an efficient activity indicator is $H_{\alpha}$, which correlates very well with the $\mathrm{S}$ index (Gomes da Silva et al. 2011). The $H_{\alpha}$ index is a well known and widely used chromospheric indicator (Kuerster et al. 2003; Bonfils et al. 2007). Further to the analysis of the $\log \left(R_{\mathrm{HK}}^{\prime}\right)$ index, we also defined a homogenized $H_{\alpha}$ index similar to Boisse et al. (2009) but using a broader central band as proposed by Pasquini \& Pallavicini (1991) in order to be more sensitive to chromospheric activity contribution (Gomes da Silva et al. 2014).

We computed the index as described in Boisse et al. (2009):

$H \alpha_{\text {index }}=\frac{F_{H \alpha}}{F_{1}+F_{2}}$,

with $F_{H \alpha}$ sampling the $H_{\alpha}$ line, and the $F_{1}$ and $F_{2}$ as the continuum on both sides of the line. The $H_{\alpha}$ line is centered on $6562.808 \AA$ with $1.6 \AA$ wide and continuum $F_{1}$ and $F_{2}$, integrated over [6545.495, 6556.245 $\AA$ ] and [6575.934, 6584.684 $\AA$ ], respectively. Each spectrum is corrected for RV shift by its RV measurement by the CCFs. The error was extracted based on the measurement of flux computed by the pipelines and using error propagation. The $H \alpha_{\text {index }}$ as defined in this way is a good indicator to measure activity variation over time for a star. However, it can not be used to compare stars with different spectral types. There is a significant correlation between $H \alpha_{\text {index }}$ and

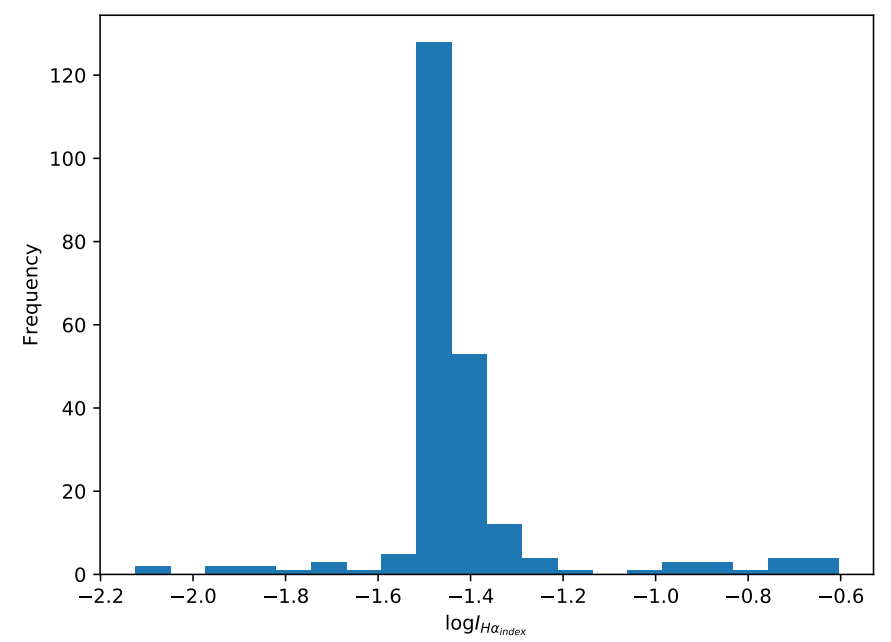

Fig. A.1. Derived $\log I_{H \alpha_{\text {index }}}$ distribution for sample stars.

$B-V$ that needs to be corrected in order to make $H \alpha_{\text {index }}$ compatible between different stars (Cincunegui et al. 2007). Here we follow a calibration extracted by Gomes da Silva et al. (2014) and extended it for the $B-V>1.2$ in order to calibrate $H \alpha_{\text {index }}$ and determine $\log I_{H \alpha_{\text {index }}}$ (see Table B.1). The average value of our calibrated $\log H_{\alpha}$ index, $\log I_{H \alpha_{\text {index }}}$ is -1.460 . As seen from Fig. A.1, most stars have values close to -1.460 , with only 15 stars having values above -1.150 . We note that 14 stars out of these "highly active stars" had already been identified and screened out using the $\log \left(R_{\mathrm{HK}}^{\prime}\right)$, which shows the strong correlation between the two indicators. 
Appendix B: Additional tables

Table B.1. Derived parameters for the first sample stars.

\begin{tabular}{|c|c|c|c|c|c|c|c|}
\hline Target & $v \sin i\left(\mathrm{~km} \mathrm{~s}^{-1}\right)$ & $\log \left(R_{\mathrm{HK}}^{\prime}\right)$ & $\log I_{H \alpha_{\text {index }}}$ & Contamination & Program ID ${ }^{(1)}$ & $V$ & Spectral-type \\
\hline HIP93203 & $25.63 \pm 0.55$ & $-4.74 \pm 0.01$ & - & $\mathrm{N}$ & {$[96],[97],[98]$} & 5.27 & G0 \\
\hline HIP115623 & $80.42 \pm 0.93$ & $-4.57 \pm 0.01$ & $-1.37 \pm 0.02$ & - & {$[96],[97],[98]$} & 4.42 & G0 \\
\hline HIP23693 & $20.16 \pm 0.35$ & $-4.33 \pm 0.01$ & $-1.44 \pm 0.03$ & $\mathrm{~N}$ & {$[88],[89]$} & 4.71 & G0 \\
\hline HIP 16852 & $3.36 \pm 0.20$ & $-5.24 \pm 0.01$ & $-1.50 \pm 0.05$ & $\mathrm{~N}$ & [39],[27] & 4.30 & G0 \\
\hline HIP1599 & $<2.0$ & $-4.89 \pm 0.01$ & $-1.48 \pm 0.06$ & $\mathrm{~N}$ & {$[81],[82]$} & 4.23 & G0 \\
\hline HIP38908 & $<2.0$ & $-4.89 \pm 0.01$ & $-1.48 \pm 0.04$ & $\mathrm{~N}$ & [81],[82] & 5.59 & G0 \\
\hline HIP77257 & $2.73 \pm 0.21$ & $-4.93 \pm 0.01$ & $-1.48 \pm 0.07$ & $\mathrm{~N}$ & {$[96],[97],[98]$} & 4.42 & G2 \\
\hline HIP22263 & $3.21 \pm 0.22$ & $-4.52 \pm 0.01$ & $-2.44 \pm 0.01$ & $\mathrm{~N}$ & {$[2],[5],[40]$} & 5.50 & $\mathrm{G} 2$ \\
\hline HIP107649 & $<2.0$ & $-5.10 \pm 0.01$ & $-1.39 \pm 0.01$ & $\mathrm{~N}$ & [43] & 5.57 & $\mathrm{G} 2$ \\
\hline HIP15371 & $<2.0$ & $-4.83 \pm 0.01$ & $-1.48 \pm 0.10$ & $\mathrm{~N}$ & [87] & 5.24 & $\mathrm{G} 2$ \\
\hline HIP58576 & $2.87 \pm 0.92$ & $-5.00 \pm 0.04$ & $-1.43 \pm 0.09$ & $\mathrm{~N}$ & [27] & 5.54 & G2 \\
\hline HIP110109 & $<2.0$ & $-4.89 \pm 0.01$ & $-1.49 \pm 0.05$ & $\mathrm{~N}$ & [82] & 5.36 & $\mathrm{G} 2$ \\
\hline HIP101916 & $4.42 \pm 0.43$ & $-5.00 \pm 0.01$ & $-1.49 \pm 0.02$ & $\mathrm{~N}$ & {$[96],[97],[98]$} & 5.07 & G5 \\
\hline HIP22449 & $19.92 \pm 2.09$ & $-4.67 \pm 0.01$ & $-1.47 \pm 0.01$ & - & {$[34],[56]$} & 3.19 & G5 \\
\hline HIP23835 & $2.36 \pm 0.31$ & - & $-1.48 \pm 0.01$ & $\mathrm{~N}$ & {$[50],[13]$} & 4.91 & G5 \\
\hline HIP95447 & - & $-5.19 \pm 0.01$ & $-1.46 \pm 0.04$ & $\mathrm{~N}$ & {$[70],[47],[23]$} & 5.17 & G5 \\
\hline HIP98767 ${ }^{(\star)}$ & $2.39 \pm 0.82$ & $-5.12 \pm 0.01$ & $-1.44 \pm 0.09$ & $\mathrm{~N}$ & [72] & 5.73 & G5 \\
\hline HIP101345 & $2.33 \pm 0.25$ & $-5.00 \pm 0.01$ & $-1.48 \pm 0.01$ & $\mathrm{~N}$ & {$[46],[11]$} & 5.66 & G5 \\
\hline HIP113357 ${ }^{(\star)}$ & $2.47 \pm 0.93$ & $-4.93 \pm 0.01$ & $-1.46 \pm 0.01$ & $\mathrm{~N}$ & [64] & 5.45 & G5 \\
\hline HIP37853 & - & $-5.09 \pm 0.01$ & $-1.47 \pm 0.02$ & $\mathrm{Y}+$ & [80] & 5.36 & G5 \\
\hline HIP86796 ${ }^{(\star)}$ & $2.95 \pm 0.90$ & $-5.15 \pm 0.01$ & $-1.37 \pm 0.07$ & $\mathrm{~N}$ & [43] & 5.12 & G5 \\
\hline HIP15330 & $<2.0$ & $-4.55 \pm 0.01$ & $-1.45 \pm 0.06$ & $\mathrm{~N}$ & {$[85],[86]$} & 5.53 & G5 \\
\hline HIP38140 & $5.14 \pm 0.24$ & $-4.89 \pm 0.01$ & $-1.50 \pm 0.01$ & $\mathrm{~N}$ & [96] & 6.55 & G8 \\
\hline HIP77512 & $5.20 \pm 0.65$ & $-4.78 \pm 0.01$ & $-1.49 \pm 0.09$ & $\mathrm{~N}$ & [98] & 4.59 & G8 \\
\hline HIP98066 & $3.39 \pm 0.61$ & $-5.00 \pm 0.01$ & $-1.47 \pm 0.05$ & $\mathrm{~N}$ & [96] & 4.70 & G8 \\
\hline HIP64924 ${ }^{(\star)}$ & $<2.0$ & $-4.91 \pm 0.01$ & $-1.45 \pm 0.01$ & $\mathrm{~N}$ & {$[46],[11]$} & 4.74 & G8 \\
\hline HIP83541 & $<2.0$ & $-5.02 \pm 0.01$ & $-1.43 \pm 0.01$ & $\mathrm{~N}$ & [11] & 6.59 & G8 \\
\hline HIP37606 & $2.49 \pm 0.34$ & $-5.15 \pm 0.01$ & $-1.47 \pm 0.01$ & $\mathrm{~N}$ & {$[61],[36]$} & 5.04 & G8 \\
\hline HIP29271 & $<2.0$ & $-4.97 \pm 0.01$ & $-1.47 \pm 0.01$ & $\mathrm{~N}$ & [69] & 5.08 & G8 \\
\hline HIP40693 ${ }^{(\star)}$ & $<2.0$ & $-4.94 \pm 0.01$ & $-1.46 \pm 0.09$ & $\mathrm{~N}$ & {$[46],[81]$} & 5.95 & G8 \\
\hline HIP57443 & $<2.0$ & $-4.88 \pm 0.01$ & $-1.49 \pm 0.06$ & $\mathrm{~N}$ & {$[46],[87]$} & 4.89 & G8 \\
\hline HIP99240 & $2.36 \pm 0.64$ & $-5.04 \pm 0.01$ & $-1.46 \pm 0.05$ & $\mathrm{~N}$ & [94] & 3.55 & G8 \\
\hline HIP22319 & $2.47 \pm 0.28$ & $-4.93 \pm 0.01$ & $-1.46 \pm 0.01$ & $\mathrm{~N}$ & {$[96],[97],[98]$} & 6.51 & K0 \\
\hline HIP83688 & $3.94 \pm 0.52$ & $-4.94 \pm 0.01$ & $-1.51 \pm 0.06$ & $\mathrm{~N}$ & {$[96],[97],[98]$} & 6.38 & K0 \\
\hline HIP43587 $\left({ }^{(\star)}\right)$ & $2.17 \pm 0.28$ & $-5.06 \pm 0.01$ & $-1.42 \pm 0.01$ & $\mathrm{~N}$ & [37] & 5.96 & K0 \\
\hline HIP41926 & - & $-4.91 \pm 0.01$ & $-1.43 \pm 0.10$ & $\mathrm{~N}$ & [43] & 6.38 & K0 \\
\hline HIP15510 & - & $-5.00 \pm 0.01$ & $-1.45 \pm 0.01$ & $\mathrm{~N}$ & [43] & 4.26 & K0 \\
\hline HIP114699 ${ }^{(\star)}$ & $<2.0$ & $-5.13 \pm 0.01$ & $-1.43 \pm 0.01$ & $\mathrm{~N}$ & [11] & 6.12 & K0 \\
\hline HIP8102 ${ }^{(\star)}$ & - & $-4.90 \pm 0.01$ & $-1.44 \pm 0.07$ & $\mathrm{~N}$ & [81] & 3.49 & K0 \\
\hline HIP81300 & $<2.0$ & $-4.51 \pm 0.01$ & $-1.43 \pm 0.08$ & $\mathrm{~N}$ & [69],[91] & 5.77 & K0 \\
\hline HIP1421 & $3.70 \pm 0.38$ & $-5.36 \pm 0.01$ & $-1.49 \pm 0.10$ & $\mathrm{~N}$ & [97] & 6.19 & K1 \\
\hline HIP6714 & $4.44 \pm 0.91$ & $-4.94 \pm 0.01$ & $-1.50 \pm 0.08$ & $\mathrm{~N}$ & [96] & 6.44 & $\mathrm{~K} 1$ \\
\hline HIP15514 & $2.99 \pm 0.28$ & $-4.96 \pm 0.01$ & $-1.48 \pm 0.03$ & $\mathrm{~N}$ & [96] & 5.92 & $\mathrm{~K} 1$ \\
\hline
\end{tabular}

Notes. ${ }^{(\star)}$ Host confirmed exoplanet, ${ }^{(1)}$ Program ID: 072.C-0488(E): [43], 072.C-0495(A): [65], 073.D-0038(D): [12], 074.B-0639(A): [66], 075.D0453(A): [72], 076.B-0055(A): [39], 076.C-0279(A): [56], 076.C-0878(B): [48], 076.D-0130(B): [18], 076.D-0130(C): [15], 076.D-0130(E): [53], 077.C-0012(A): [21], 077.C-0364(E): [63], 078.C-0209(B): [34], 078.C-0751(B): [36], 078.C-0833(A): [3], 078.D-0760(B): [60], 079.C-0046(A): [68], 079.C-0521(A): [25], 079.C-0657(C): [17], 079.C-0681(A): [45], 079.D-0075(A): [32], 079.D-0466(A): [29], 080.D-0086(B): [78], 080.D0086(C): [71], 080.D-0347(A): [26], 081.C-0802(B): [61], 081.C-0802(C): [4], 081.D-0065(E): [16], 082.C-0427(A): [51], 082.C-0427(C): [58], 082.C-0718(B): [22], 082.D-0953(A): [62], 084.C-0229(A): [35], 084.C-0403(A): [24], 085.C-0019(A): [67], 086.C-0145(A): [13], 086.C-0284(A): [33], 087.C-0831(A): [57], 090.C-0421(A): [59], 091.C-0271(A): [64], 092.C-0721(A): [41], 092.D-0207(A): [77], 093.C-0062(A): [55], 093.C0343(A): [14], 093.C-0409(A): [19], 094.C-0322(A): [50], 094.C-0367(A): [54], 094.D-0596(A): [8], 095.C-0551(A): [1], 096.C-0708(A): [73], 173.C-0606(B): [74], 180.C-0886(A): [75], 183.C-0437(A): [31], 183.C-0972(A): [11], 184.C-0815(C): [28], 188.C-0265(B): [5], 188.C-0265(M): [40], 188.C-0265(O): [2], 191.C-0873: [10], 191.C-0873(A): [6], 192.C-0224: [9], 192.C-0224(B): [42], 192.C-0224(C): [76], 192.C-0224(G): [52], 192.C-0224(H): [44], 192.C-0852(A): [46], 266.D-5655(A): [27], 288.C-5010(A): [37], 289.C-5053(A): [20], 290.C-5196(A): [47], 290.C-5196(B): [23], 380.C-0083(A): [38], 60.A-9036(A): [69], 67.D-0321(B): [70], 68.D-0166(A): [30], 71.C-0498(A): [49], Lagrange: [7], '077.C-0530(A)': [79], '076.D-0103(A)': [80], '198.C-0836(A)': [81], '097.C-0021(A)': [82], '60.A-9700(G)': [83], '073.D-0038(C)': [84], '074.C-0012(A)': [85], '074.C-0012(B)': [86], '196.C-1006(A)': [87], '184.C-0815(A)': [88], '184.C-0815(F)': [89], '072.D-0707(A)': [90], '088.C-0513(B)': [91], '495.L0963(A)': [92], '096.C-0460(A)': [93], '082.C-0315(A)': [94], '099.C-0880(A)': [95], '97.C-0561(A)': [96], '97.C-0561(B)': [97], A33T AC_7: [98].

A80, page 16 of 27 
Table B.1. continued.

\begin{tabular}{|c|c|c|c|c|c|c|c|}
\hline Target & $v \sin i\left(\mathrm{~km} \mathrm{~s}^{-1}\right)$ & $\log \left(R_{\mathrm{HK}}^{\prime}\right)$ & $\log I_{H \alpha_{\text {index }}}$ & Contamination & Program ID ${ }^{(2)}$ & $V$ & Spectral-type \\
\hline HIP16641 & $2.29 \pm 0.72$ & $-5.29 \pm 0.03$ & $-1.46 \pm 0.04$ & $\mathrm{Y}$ & {$[96]$} & 6.17 & $\mathrm{~K} 1$ \\
\hline HIP17027 & $2.29 \pm 0.24$ & $-5.21 \pm 0.03$ & $-1.45 \pm 0.10$ & $\mathrm{~N}$ & {$[96],[97],[98]$} & 5.96 & $\mathrm{~K} 1$ \\
\hline HIP27280 & $3.71 \pm 0.53$ & $-5.19 \pm 0.03$ & $-1.51 \pm 0.02$ & $\mathrm{~N}$ & {$[96],[97],[98]$} & 5.78 & $\mathrm{~K} 1$ \\
\hline HIP30815 & $2.82 \pm 0.21$ & $-5.26 \pm 0.02$ & $-1.48 \pm 0.05$ & $\mathrm{Y}$ & {$[96],[97],[98]$} & 6.24 & $\mathrm{~K} 1$ \\
\hline HIP38303 & $5.34 \pm 0.30$ & $-5.13 \pm 0.01$ & $-1.53 \pm 0.06$ & $\mathrm{~N}$ & [96] & 6.49 & $\mathrm{~K} 1$ \\
\hline HIP38375 & $3.85 \pm 0.55$ & $-4.94 \pm 0.01$ & $-1.50 \pm 0.06$ & $\mathrm{~N}$ & [96] & 5.62 & $\mathrm{~K} 1$ \\
\hline HIP46543 & $2.02 \pm 0.47$ & $-5.15 \pm 0.01$ & $-1.49 \pm 0.10$ & $\mathrm{~N}$ & {$[96],[97],[98]$} & 6.25 & $\mathrm{~K} 1$ \\
\hline HIP50887 ${ }^{(\star)}$ & $2.85 \pm 0.23$ & $-5.28 \pm 0.01$ & $-1.47 \pm 0.06$ & $\mathrm{Y}$ & {$[96],[97],[98]$} & 6.45 & $\mathrm{~K} 1$ \\
\hline HIP56830 & $<2.0$ & $-5.14 \pm 0.01$ & $-1.49 \pm 0.04$ & $\mathrm{~N}$ & [96] & 6.40 & $\mathrm{~K} 1$ \\
\hline HIP62103 & $2.34 \pm 0.24$ & $-5.20 \pm 0.01$ & $-1.49 \pm 0.09$ & Y & {$[96],[97],[98]$} & 5.91 & $\mathrm{~K} 1$ \\
\hline HIP70038 & $4.44 \pm 0.32$ & $-5.07 \pm 0.01$ & $-1.50 \pm 0.06$ & $\mathrm{~N}$ & {$[96],[97],[98]$} & 6.42 & K1 \\
\hline HIP70336 & $3.94 \pm 0.42$ & $-5.40 \pm 0.01$ & $-1.48 \pm 0.09$ & $\mathrm{~N}$ & {$[96],[97],[98]$} & 6.22 & $\mathrm{~K} 1$ \\
\hline HIP108868 & $4.36 \pm 0.61$ & $-5.15 \pm 0.01$ & $-1.50 \pm 0.01$ & $\mathrm{~N}$ & {$[96],[97],[98]$} & 5.55 & $\mathrm{~K} 1$ \\
\hline HIP50903 & $2.48 \pm 0.24$ & $-5.23 \pm 0.01$ & $-1.48 \pm 0.03$ & $\mathrm{~N}$ & [96] & 6.24 & $\mathrm{~K} 1$ \\
\hline HIP38183 & $2.90 \pm 0.27$ & $-5.31 \pm 0.01$ & $-1.51 \pm 0.02$ & $\mathrm{~N}$ & [96] & 6.32 & K1 \\
\hline HIP33139 & $2.61 \pm 0.48$ & $-5.11 \pm 0.01$ & $-1.47 \pm 0.06$ & $\mathrm{~N}$ & [96] & 6.24 & $\mathrm{~K} 1$ \\
\hline HIP57137 & $2.10 \pm 0.75$ & $-5.22 \pm 0.01$ & $-1.48 \pm 0.07$ & $\mathrm{~N}$ & [96] & 6.38 & $\mathrm{~K} 1$ \\
\hline HIP3834(^) & - & - & $-1.48 \pm 0.03$ & $\mathrm{~N}$ & [54] & 5.90 & $\mathrm{~K} 1$ \\
\hline HIP13402 & $4.68 \pm 0.21$ & $-4.37 \pm 0.01$ & $-1.34 \pm 0.01$ & $\mathrm{~N}$ & {$[9],[76],[7]$} & 6.05 & $\mathrm{~K} 1$ \\
\hline HIP16537 ${ }^{(\star)}$ & $2.17 \pm 0.25$ & $-4.47 \pm 0.01$ & $-2.68 \pm 0.01$ & $\mathrm{~N}$ & [69] & 3.73 & $\mathrm{~K} 1$ \\
\hline HIP17378 & $2.11 \pm 0.26$ & $-5.20 \pm 0.01$ & $-1.45 \pm 0.01$ & $\mathrm{~N}$ & [43] & 3.54 & $\mathrm{~K} 1$ \\
\hline HIP19849 & $<2.0$ & $-4.92 \pm 0.01$ & $-1.41 \pm 0.01$ & $\mathrm{~N}$ & {$[78],[16]$} & 4.43 & K1 \\
\hline HIP35846 & - & $-5.19 \pm 0.01$ & $-1.50 \pm 0.01$ & $\mathrm{~N}$ & [66] & 5.04 & $\mathrm{~K} 1$ \\
\hline HIP52316 & $2.11 \pm 0.37$ & $-5.08 \pm 0.01$ & $-1.46 \pm 0.01$ & $\mathrm{~N}$ & [43] & 6.25 & $\mathrm{~K} 1$ \\
\hline HIP85207 & $4.27 \pm 0.36$ & $-4.92 \pm 0.01$ & $-2.81 \pm 0.01$ & $\mathrm{~N}$ & {$[58],[4],[61]$} & 5.82 & $\mathrm{~K} 1$ \\
\hline HIP99825 ${ }^{(\star)}$ & $<2.0$ & $-4.96 \pm 0.01$ & $-1.41 \pm 0.01$ & $\mathrm{~N}$ & {$[55],[35]$} & 5.73 & $\mathrm{~K} 1$ \\
\hline HIP99894 ${ }^{(\star)}$ & $<2.0$ & - & $-1.48 \pm 0.01$ & $\mathrm{~N}$ & [38] & 6.44 & K1 \\
\hline HIP109577 $\left.{ }^{\star}{ }^{\star}\right)$ & $2.50 \pm 0.48$ & - & $-1.46 \pm 0.03$ & $\mathrm{~N}$ & [38] & 5.93 & $\mathrm{~K} 1$ \\
\hline HIP10138 ${ }^{(\star)}$ & $<2.0$ & $-4.69 \pm 0.02$ & $-1.43 \pm 0.09$ & $\mathrm{~N}$ & [83] & 6.12 & K1 \\
\hline HIP56452 & - & $-4.83 \pm 0.66$ & $-1.44 \pm 0.10$ & $\mathrm{~N}$ & {$[43],[90]$} & 5.96 & $\mathrm{~K} 1$ \\
\hline HIP6605 & $2.88 \pm 0.47$ & $-5.32 \pm 0.01$ & $-1.49 \pm 0.08$ & $\mathrm{~N}$ & [96] & 6.17 & $\mathrm{~K} 2$ \\
\hline HIP10446 & $3.03 \pm 0.74$ & $-5.59 \pm 0.23$ & $-1.46 \pm 0.02$ & $\mathrm{Y}$ & [96] & 6.35 & $\mathrm{~K} 2$ \\
\hline HIP17086 & $3.87 \pm 0.32$ & $-5.48 \pm 0.01$ & $-1.51 \pm 0.03$ & $\mathrm{~N}$ & [96] & 6.22 & $\mathrm{~K} 2$ \\
\hline HIP24130 & $2.47 \pm 0.23$ & $-5.33 \pm 0.01$ & $-1.46 \pm 0.09$ & $\mathrm{~N}$ & {$[96],[97],[98]$} & 6.24 & $\mathrm{~K} 2$ \\
\hline HIP24679 & $2.28 \pm 0.31$ & $-5.28 \pm 0.01$ & $-1.49 \pm 0.06$ & $\mathrm{~N}$ & {$[96],[97],[98]$} & 5.48 & $\mathrm{~K} 2$ \\
\hline HIP26366 & $2.47 \pm 0.63$ & $-5.31 \pm 0.01$ & $-1.50 \pm 0.07$ & $\mathrm{~N}$ & {$[96],[97],[98]$} & 4.09 & $\mathrm{~K} 2$ \\
\hline HIP31674 & $3.25 \pm 0.46$ & $-5.34 \pm 0.01$ & $-1.50 \pm 0.05$ & $\mathrm{~N}$ & [96] & 6.11 & $\mathrm{~K} 2$ \\
\hline HIP38748 & $2.58 \pm 0.29$ & $-5.28 \pm 0.01$ & $-1.49 \pm 0.06$ & $\mathrm{~N}$ & [96] & 6.57 & $\mathrm{~K} 2$ \\
\hline HIP50939 & $2.76 \pm 0.37$ & $-5.34 \pm 0.01$ & $-1.50 \pm 0.03$ & $\mathrm{~N}$ & {$[96],[97],[98]$} & 6.33 & $\mathrm{~K} 2$ \\
\hline HIP57079 & $2.74 \pm 0.27$ & $-5.31 \pm 0.01$ & $-1.48 \pm 0.09$ & $\mathrm{~N}$ & [96] & 6.21 & $\mathrm{~K} 2$ \\
\hline HIP62325 & $2.59 \pm 0.25$ & $-5.32 \pm 0.01$ & $-1.47 \pm 0.02$ & $\mathrm{~N}$ & {$[96],[97],[98]$} & 5.65 & $\mathrm{~K} 2$ \\
\hline HIP64725 & $2.75 \pm 0.20$ & $-5.38 \pm 0.01$ & $-1.47 \pm 0.07$ & $\mathrm{Y}$ & {$[96],[97],[98]$} & 5.21 & $\mathrm{~K} 2$ \\
\hline HIP64751 & $3.19 \pm 0.36$ & $-5.31 \pm 0.01$ & $-1.49 \pm 0.02$ & $\mathrm{~N}$ & {$[96],[97],[98]$} & 6.46 & $\mathrm{~K} 2$ \\
\hline HIP79137 & $2.27 \pm 0.27$ & $-5.31 \pm 0.01$ & $-1.43 \pm 0.08$ & $\mathrm{~N}$ & {$[96],[97],[98]$} & 5.93 & $\mathrm{~K} 2$ \\
\hline HIP79522 & $3.03 \pm 0.25$ & $-5.36 \pm 0.01$ & $-1.50 \pm 0.05$ & $\mathrm{Y}$ & {$[96],[97],[98]$} & 6.45 & $\mathrm{~K} 2$ \\
\hline HIP82989 & $2.68 \pm 0.21$ & $-5.27 \pm 0.01$ & $-1.46 \pm 0.08$ & $\mathrm{~N}$ & {$[96],[97],[98]$} & 6.38 & $\mathrm{~K} 2$ \\
\hline HIP87834 & $2.45 \pm 0.26$ & $-5.30 \pm 0.01$ & $-1.43 \pm 0.07$ & $\mathrm{~N}$ & {$[96],[97],[98]$} & 6.55 & $\mathrm{~K} 2$ \\
\hline HIP94521 & $2.57 \pm 0.20$ & $-5.32 \pm 0.01$ & $-1.47 \pm 0.04$ & $\mathrm{~N}$ & {$[96],[97],[98]$} & 6.54 & $\mathrm{~K} 2$ \\
\hline HIP99913 & $3.38 \pm 0.22$ & $-5.44 \pm 0.01$ & $-1.48 \pm 0.10$ & $\mathrm{~N}$ & {$[96],[97],[98]$} & 6.11 & $\mathrm{~K} 2$ \\
\hline HIP108513 & $2.95 \pm 0.23$ & $-5.30 \pm 0.01$ & $-1.46 \pm 0.02$ & $\mathrm{~N}$ & {$[96],[97],[98]$} & 6.50 & $\mathrm{~K} 2$ \\
\hline HIP113801 & $3.00 \pm 0.74$ & $-5.33 \pm 0.01$ & $-1.49 \pm 0.08$ & $\mathrm{~N}$ & [96] & 5.97 & $\mathrm{~K} 2$ \\
\hline HIP114526 & $2.80 \pm 0.21$ & $-5.29 \pm 0.01$ & $-1.47 \pm 0.01$ & $\mathrm{~N}$ & {$[96],[97],[98]$} & 6.19 & $\mathrm{~K} 2$ \\
\hline HIP67851 ${ }^{(\star)}$ & $2.38 \pm 0.51$ & $-5.36 \pm 0.01$ & $-1.47 \pm 0.07$ & $\mathrm{~N}$ & [96] & 6.17 & $\mathrm{~K} 2$ \\
\hline HIP42808 & $3.72 \pm 0.84$ & $-4.42 \pm 0.01$ & $-1.34 \pm 0.03$ & $\mathrm{~N}$ & [96] & 6.58 & $\mathrm{~K} 2$ \\
\hline HIP3765 & - & $-4.93 \pm 0.01$ & $-1.41 \pm 0.01$ & $\mathrm{~N}$ & [73] & 5.74 & $\mathrm{~K} 2$ \\
\hline HIP18606 & $3.24 \pm 0.54$ & $-5.46 \pm 0.01$ & $-1.47 \pm 0.02$ & $\mathrm{~N}$ & [27] & 5.83 & $\mathrm{~K} 2$ \\
\hline HIP37826 ${ }^{(\star)}$ & $3.29 \pm 0.21$ & $-5.09 \pm 0.09$ & $-1.48 \pm 0.01$ & $\mathrm{~N}$ & {$[77],[38]$} & 1.16 & $\mathrm{~K} 2$ \\
\hline HIP76219 & $2.27 \pm 0.27$ & $-4.50 \pm 0.01$ & $-1.47 \pm 0.01$ & $\mathrm{~N}$ & {$[21],[68]$} & 4.61 & $\mathrm{~K} 2$ \\
\hline HIP86400 & $<2.0$ & $-4.76 \pm 0.01$ & $-1.38 \pm 0.01$ & $\mathrm{Y}$ & {$[53],[12],[32]$} & 6.53 & $\mathrm{~K} 2$ \\
\hline HIP89962 & $2.65 \pm 0.21$ & $-5.01 \pm 0.01$ & $-1.49 \pm 0.01$ & $\mathrm{~N}$ & [29] & 3.23 & $\mathrm{~K} 2$ \\
\hline
\end{tabular}


Table B.1. continued.

\begin{tabular}{|c|c|c|c|c|c|c|c|}
\hline Target & $v \sin i\left(\mathrm{~km} \mathrm{~s}^{-1}\right)$ & $\log \left(R_{H K}^{\prime}\right)$ & $\log I_{H \alpha_{\text {index }}}$ & Contamination & Program ID ${ }^{(1)}$ & $V$ & Spectral-type \\
\hline HIP109822 & $<2.0$ & $-5.18 \pm 0.01$ & $-1.44 \pm 0.01$ & $\mathrm{~N}$ & {$[43],[46]$} & 6.55 & $\mathrm{~K} 2$ \\
\hline HIP24659 & $2.52 \pm 0.41$ & $-5.31 \pm 0.01$ & $-1.42 \pm 0.08$ & $\mathrm{~N}$ & {$[17],[36]$} & 4.81 & $\mathrm{~K} 2$ \\
\hline HIP99461 & $<2.0$ & - & $-1.41 \pm 0.02$ & $\mathrm{~N}$ & {$[45],[79]$} & 5.32 & $\mathrm{~K} 2$ \\
\hline HIP21253 & $2.48 \pm 0.74$ & $-5.36 \pm 0.01$ & $-1.48 \pm 0.05$ & $\mathrm{~N}$ & {$[17],[51]$} & 5.79 & $\mathrm{~K} 2$ \\
\hline HIP12114 & $2.00 \pm 0.27$ & $-4.96 \pm 0.01$ & $-1.38 \pm 0.04$ & $\mathrm{~N}$ & {$[15],[84]$} & 5.79 & $\mathrm{~K} 2$ \\
\hline HIP9862 & $3.36 \pm 0.21$ & $-5.59 \pm 0.01$ & $-1.49 \pm 0.07$ & $\mathrm{~N}$ & [96] & 6.39 & K3 \\
\hline HIP67890 & $3.38 \pm 0.66$ & $-5.56 \pm 0.01$ & $-1.46 \pm 0.10$ & $\mathrm{~N}$ & [96] & 6.05 & K3 \\
\hline HIP73927 & $2.79 \pm 0.42$ & $-5.41 \pm 0.01$ & $-1.48 \pm 0.03$ & $\mathrm{~N}$ & {$[96]$} & 6.13 & K3 \\
\hline HIP79605 & $3.14 \pm 0.21$ & $-5.44 \pm 0.01$ & $-1.49 \pm 0.06$ & $\mathrm{~N}$ & {$[96],[97],[98]$} & 6.32 & $\mathrm{~K} 3$ \\
\hline HIP87540 & $3.24 \pm 0.27$ & $-5.50 \pm 0.01$ & $-1.51 \pm 0.02$ & $\mathrm{~N}$ & {$[96],[97],[98]$} & 6.17 & K3 \\
\hline HIP98920 & $3.23 \pm 0.74$ & $-5.40 \pm 0.01$ & $-1.48 \pm 0.06$ & $\mathrm{~N}$ & {$[96],[97],[98]$} & 5.09 & K3 \\
\hline HIP99171 & $2.53 \pm 0.20$ & $-5.37 \pm 0.01$ & $-1.46 \pm 0.09$ & $\mathrm{~N}$ & {$[96],[97],[98]$} & 5.97 & K3 \\
\hline HIP101221 & $3.63 \pm 0.42$ & $-4.85 \pm 0.01$ & $-1.45 \pm 0.03$ & $\mathrm{Y}$ & {$[96],[97],[98]$} & 6.10 & $\mathrm{~K} 3$ \\
\hline HIP102891 & $3.09 \pm 0.45$ & $-5.45 \pm 0.01$ & $-1.51 \pm 0.01$ & $\mathrm{~N}$ & {$[96],[97],[98]$} & 5.87 & K3 \\
\hline HIP113148 & $2.91 \pm 0.28$ & $-5.51 \pm 0.01$ & $-1.46 \pm 0.07$ & $\mathrm{~N}$ & {$[96],[97],[98]$} & 5.53 & $\mathrm{~K} 3$ \\
\hline HIP115953 & $3.03 \pm 0.62$ & $-5.45 \pm 0.01$ & $-1.43 \pm 0.01$ & $\mathrm{~N}$ & {$[96],[97],[98]$} & 6.26 & K3 \\
\hline HIP116624 & $3.77 \pm 0.33$ & $-5.50 \pm 0.01$ & $-1.52 \pm 0.02$ & $\mathrm{Y}$ & {$[96],[97],[98]$} & 6.38 & K3 \\
\hline HIP45002 & $2.66 \pm 0.75$ & $-5.35 \pm 0.01$ & $-1.50 \pm 0.02$ & $\mathrm{~N}$ & [96] & 6.19 & K3 \\
\hline HIP62268 & $2.78 \pm 0.97$ & $-5.38 \pm 0.01$ & $-1.47 \pm 0.07$ & $\mathrm{~N}$ & [96] & 4.69 & K3 \\
\hline HIP23311 & $<2.0$ & $-5.00 \pm 0.01$ & $-1.39 \pm 0.01$ & $\mathrm{~N}$ & {$[71],[18],[15]$} & 6.22 & $\mathrm{~K} 3$ \\
\hline HIP31592 $2^{(\star)}$ & $2.72 \pm 0.21$ & $-5.41 \pm 0.01$ & $-3.60 \pm 0.01$ & $\mathrm{~N}$ & {$[36],[51]$} & 3.95 & $\mathrm{~K} 3$ \\
\hline HIP32984 & $2.31 \pm 0.55$ & - & $-1.20 \pm 0.04$ & $\mathrm{~N}$ & [43] & 6.58 & K3 \\
\hline HIP80910 & $2.92 \pm 0.20$ & $-5.44 \pm 0.01$ & - & $\mathrm{N}$ & {$[17],[61]$} & 6.07 & $\mathrm{~K} 3$ \\
\hline HIP4801 & $3.53 \pm 0.20$ & $-5.67 \pm 0.01$ & $-1.48 \pm 0.02$ & $\mathrm{~N}$ & [96] & 6.47 & K4 \\
\hline HIP12754 & $3.38 \pm 0.21$ & $-5.64 \pm 0.01$ & - & $\mathrm{N}$ & [96] & 6.54 & K4 \\
\hline HIP30804 & $3.31 \pm 0.20$ & $-5.53 \pm 0.02$ & $-1.48 \pm 0.07$ & $\mathrm{~N}$ & {$[96],[97],[98]$} & 6.16 & K4 \\
\hline HIP53259 & $3.37 \pm 0.21$ & $-5.67 \pm 0.01$ & $-1.48 \pm 0.08$ & $\mathrm{~N}$ & [96] & 6.34 & K4 \\
\hline HIP54863 & $3.22 \pm 0.30$ & $-5.51 \pm 0.01$ & $-1.49 \pm 0.09$ & $\mathrm{~N}$ & [96] & 5.79 & K4 \\
\hline HIP96229 & $3.27 \pm 0.49$ & $-5.58 \pm 0.01$ & $-1.49 \pm 0.06$ & Y & {$[96],[97],[98]$} & 4.45 & K4 \\
\hline HIP116422 & $3.36 \pm 0.20$ & $-5.55 \pm 0.01$ & $-1.51 \pm 0.05$ & $\mathrm{Y}$ & {$[96],[97],[98]$} & 6.47 & K4 \\
\hline HIP49844 & $3.24 \pm 0.47$ & $-5.66 \pm 0.01$ & $-1.49 \pm 0.10$ & $\mathrm{~N}$ & [96] & 5.98 & K4 \\
\hline HIP38267 & $2.75 \pm 0.71$ & $-5.41 \pm 0.01$ & $-1.50 \pm 0.09$ & $\mathrm{~N}$ & [96] & 5.89 & K4 \\
\hline HIP33588 & $2.96 \pm 0.28$ & $-5.53 \pm 0.01$ & $-1.50 \pm 0.04$ & $\mathrm{~N}$ & [96] & 6.26 & K4 \\
\hline HIP113283 & $2.71 \pm 0.28$ & $-4.48 \pm 0.01$ & $-1.30 \pm 0.01$ & $\mathrm{~N}$ & [69] & 6.48 & K4 \\
\hline HIP16711 & $<2.0$ & $-5.09 \pm 0.01$ & $-1.52 \pm 0.01$ & $\mathrm{~N}$ & {$[67],[43]$} & 8.57 & K4 \\
\hline HIP108870 & $<2.0$ & $-4.80 \pm 0.01$ & $-1.34 \pm 0.07$ & $\mathrm{~N}$ & {$[43],[46]$} & 4.69 & K4 \\
\hline HIP84478 & $<2.0$ & $-4.66 \pm 0.01$ & $-1.37 \pm 0.06$ & $\mathrm{~N}$ & [92] & 6.33 & K4 \\
\hline HIP92444 & $<2.0$ & $-4.71 \pm 0.01$ & $-1.56 \pm 0.06$ & $\mathrm{~N}$ & [43] & 9.65 & K5 \\
\hline HIP20917 & $4.71 \pm 0.26$ & $-4.80 \pm 0.01$ & $-1.48 \pm 0.01$ & $\mathrm{~N}$ & {$[96],[97],[98]$} & 8.30 & K7 \\
\hline HIP85647 ${ }^{(\star)}$ & $2.64 \pm 0.36$ & $-5.12 \pm 0.02$ & $-1.53 \pm 0.04$ & $\mathrm{~N}$ & [11] & 9.58 & K7 \\
\hline PM J18548+1058 & - & $-4.98 \pm 0.08$ & $-1.55 \pm 0.02$ & $\mathrm{~N}$ & [25] & 9.62 & K7 \\
\hline PM J02239+1812 & $6.02 \pm 0.48$ & $-5.51 \pm 0.09$ & $-1.64 \pm 0.02$ & $\mathrm{~N}$ & [97] & 9.23 & M0 \\
\hline PM J03219+0940 & $4.92 \pm 0.20$ & $-5.35 \pm 0.29$ & $-2.08 \pm 0.04$ & $\mathrm{~N}$ & [97] & 8.99 & M0 \\
\hline PM J03340+1123 & $4.51 \pm 0.21$ & $-5.72 \pm 0.20$ & $-1.59 \pm 0.08$ & $\mathrm{~N}$ & [97] & 9.61 & M0 \\
\hline HIP40239 & $4.89 \pm 0.23$ & $-5.65 \pm 0.25$ & $-1.39 \pm 0.07$ & $\mathrm{~N}$ & [97] & 9.38 & M0 \\
\hline HIP66675 & $2.46 \pm 0.35$ & $-5.18 \pm 0.04$ & $-1.44 \pm 0.04$ & $\mathrm{Y}$ & {$[98],[97]$} & 9.61 & M0 \\
\hline PM J15357-2812 & $2.21 \pm 0.74$ & $-5.05 \pm 0.11$ & $-1.94 \pm 0.07$ & $\mathrm{~N}$ & [97] & 8.76 & M0 \\
\hline PM J16015-4710 & $2.70 \pm 0.57$ & $-5.60 \pm 0.08$ & $-1.88 \pm 0.06$ & $\mathrm{~N}$ & [97] & 9.41 & M0 \\
\hline HIP78508 & $2.51 \pm 0.62$ & $-5.68 \pm 0.10$ & $-1.72 \pm 0.06$ & $\mathrm{~N}$ & [97] & 9.20 & M0 \\
\hline HIP79148 & $3.58 \pm 0.44$ & $-5.71 \pm 0.11$ & $-1.92 \pm 0.04$ & $\mathrm{~N}$ & [97] & 9.25 & M0 \\
\hline PM J17173+0003 & $4.25 \pm 0.50$ & $-5.46 \pm 0.11$ & $-1.88 \pm 0.06$ & $\mathrm{~N}$ & [97] & 9.50 & M0 \\
\hline HIP73457 & $<2.0$ & $-4.66 \pm 0.01$ & $-1.47 \pm 0.02$ & $\mathrm{~N}$ & {$[43],[11]$} & 9.49 & M0 \\
\hline HIP8768 & $<2.0$ & $-4.74 \pm 0.01$ & $-1.48 \pm 0.02$ & $\mathrm{Y}$ & [1] & 8.89 & M0 \\
\hline HIP17609 & $2.71 \pm 0.34$ & - & - & $\mathrm{N}$ & [8] & 9.61 & M0 \\
\hline HIP37288 & $<2.0$ & $-4.64 \pm 0.02$ & $-1.47 \pm 0.02$ & $\mathrm{~N}$ & {$[42],[76]$} & 9.66 & M0 \\
\hline HIP44722 & $<2.0$ & $-4.65 \pm 0.01$ & $-1.41 \pm 0.02$ & $\mathrm{~N}$ & {$[44],[52]$} & 9.50 & M0 \\
\hline HIP62687 & $2.75 \pm 0.36$ & $-4.68 \pm 0.01$ & $-1.36 \pm 0.02$ & $\mathrm{~N}$ & [43] & 8.49 & M0 \\
\hline HIP70956 & $<2.0$ & $-4.90 \pm 0.01$ & $-1.51 \pm 0.02$ & $\mathrm{~N}$ & [43] & 9.40 & M0 \\
\hline HIP2540 & $2.17 \pm 0.32$ & $-4.62 \pm 0.04$ & $-1.47 \pm 0.06$ & $\mathrm{~N}$ & [43] & 9.65 & M0 \\
\hline HIP25878 & $2.50 \pm 0.29$ & $-4.75 \pm 0.02$ & $-1.42 \pm 0.01$ & $\mathrm{~N}$ & [43] & 7.97 & M0 \\
\hline
\end{tabular}

A80, page 18 of 27 
Table B.1. continued.

\begin{tabular}{|c|c|c|c|c|c|c|c|}
\hline Target & $v \sin i\left(\mathrm{~km} \mathrm{~s}^{-1}\right)$ & $\log \left(R_{\mathrm{HK}}^{\prime}\right)$ & $\log I_{H \alpha_{\text {index }}}$ & Contamination & Program ID ${ }^{(2)}$ & $V$ & Spectral-type \\
\hline PM J03377+1042 & $4.21 \pm 0.52$ & $-5.46 \pm 0.11$ & $-1.75 \pm 0.02$ & $\mathrm{~N}$ & [97] & 9.65 & M1 \\
\hline HIP31634 & $<2.0$ & $-4.87 \pm 0.05$ & - & $\mathrm{N}$ & [97] & 9.61 & M1 \\
\hline HIP42748 & $2.67 \pm 0.72$ & $-5.48 \pm 0.10$ & $-1.39 \pm 0.02$ & $\mathrm{~N}$ & [97] & 9.62 & M1 \\
\hline HIP56244 & $17.41 \pm 2.12$ & $-4.27 \pm 0.07$ & $-0.62 \pm 0.05$ & $\mathrm{~N}$ & [97] & 11.55 & M1 \\
\hline PM J15543-3536 & $4.12 \pm 0.94$ & $-5.73 \pm 0.16$ & - & $\mathrm{N}$ & [97] & 9.32 & M1 \\
\hline PM J16046-5521 & $3.87 \pm 0.82$ & $-5.72 \pm 0.09$ & $-2.13 \pm 0.07$ & $\mathrm{~N}$ & [97] & 9.24 & M1 \\
\hline PM J16051-0508 & $4.39 \pm 0.37$ & $-5.61 \pm 0.10$ & $-1.36 \pm 0.09$ & $\mathrm{~N}$ & [97] & 9.48 & M1 \\
\hline HIP81705 & $3.10 \pm 0.66$ & $-5.73 \pm 0.29$ & $-1.68 \pm 0.05$ & $\mathrm{~N}$ & [97] & 8.99 & M1 \\
\hline PM J16565-4749 & - & $-5.78 \pm 0.12$ & - & $\mathrm{N}$ & [97] & 9.43 & M1 \\
\hline PM J18400-1644 & $2.10 \pm 0.89$ & $-5.55 \pm 0.09$ & $-1.68 \pm 0.04$ & $\mathrm{~N}$ & [97] & 9.30 & M1 \\
\hline HIP93069 & $<2.0$ & $-5.19 \pm 0.01$ & - & $\mathrm{N}$ & [97] & 8.86 & M1 \\
\hline HIP94739 & - & $-4.85 \pm 0.03$ & $-1.41 \pm 0.03$ & $\mathrm{Y}+$ & [97] & 9.38 & M1 \\
\hline PM J19473-2424 & $3.89 \pm 0.40$ & $-5.49 \pm 0.10$ & $-1.33 \pm 0.10$ & $\mathrm{~N}$ & [97] & 9.65 & M1 \\
\hline HIP439 & $<2.0$ & $-5.47 \pm 0.01$ & $-1.36 \pm 0.02$ & $\mathrm{~N}$ & [35] & 8.56 & M1 \\
\hline HIP23452 & $4.23 \pm 0.29$ & $-5.42 \pm 0.02$ & $-1.42 \pm 0.04$ & $\mathrm{~N}$ & [43] & 8.31 & M1 \\
\hline HIP26335 & $<2.0$ & $-4.36 \pm 0.01$ & $-1.22 \pm 0.02$ & $\mathrm{~N}$ & [76] & 9.02 & M1 \\
\hline HIP29295 & $2.61 \pm 0.41$ & $-4.90 \pm 0.01$ & $-1.51 \pm 0.02$ & $\mathrm{~N}$ & {$[10],[6]$} & 8.15 & M1 \\
\hline HIP45908 & $2.32 \pm 0.65$ & $-4.94 \pm 0.01$ & $-1.46 \pm 0.02$ & $\mathrm{~N}$ & [33] & 9.49 & M1 \\
\hline PM J13477-3225E & $2.08 \pm 0.48$ & $-5.17 \pm 0.03$ & $-1.49 \pm 0.03$ & $\mathrm{~N}$ & [44], [52], [76] & 9.20 & M1 \\
\hline HIP85665 & $2.51 \pm 0.21$ & $-4.91 \pm 0.01$ & $-1.42 \pm 0.02$ & $\mathrm{Y}$ & {$[31],[43],[6]$} & 9.33 & M1 \\
\hline HIP93101 & $2.44 \pm 0.65$ & $-4.86 \pm 0.01$ & $-1.46 \pm 0.02$ & $\mathrm{~N}$ & {$[25],[57]$} & 9.22 & M1 \\
\hline HIP108782 & $2.65 \pm 0.27$ & $-4.83 \pm 0.01$ & $-1.47 \pm 0.05$ & $\mathrm{~N}$ & {$[25],[35]$} & 9.17 & M1 \\
\hline HIP113296 & $2.62 \pm 0.69$ & $-4.98 \pm 0.01$ & $-1.51 \pm 0.02$ & $\mathrm{Y}$ & {$[25],[35],[6]$} & 8.68 & M1 \\
\hline HIP117473 & $2.40 \pm 0.20$ & $-5.47 \pm 0.01$ & $-1.40 \pm 0.02$ & $\mathrm{~N}$ & {$[25],[35],[50]$} & 8.98 & M1 \\
\hline HIP113576 & $<2.0$ & $-4.61 \pm 0.01$ & $-1.48 \pm 0.02$ & $\mathrm{~N}$ & {$[43],[93]$} & 7.88 & M1 \\
\hline HIP107705 & $5.15 \pm 0.42$ & $-4.23 \pm 0.01$ & $-0.94 \pm 0.10$ & $\mathrm{~N}$ & {$[96],[97],[98]$} & 9.53 & M2 \\
\hline HIP23708 & $<2.0$ & $-4.96 \pm 0.01$ & $-1.42 \pm 0.05$ & $\mathrm{~N}$ & [43] & 9.11 & M2 \\
\hline HIP24186 ${ }^{(\star)}$ & $3.01 \pm 0.78$ & $-5.10 \pm 0.06$ & $-1.43 \pm 0.03$ & $\mathrm{~N}$ & [43] & 8.86 & $\mathrm{M} 2$ \\
\hline HIP49986 & $2.48 \pm 0.21$ & $-4.83 \pm 0.01$ & $-1.39 \pm 0.02$ & $\mathrm{~N}$ & [43] & 9.26 & M2 \\
\hline HIP51317 & $2.28 \pm 0.20$ & $-5.16 \pm 0.02$ & $-1.41 \pm 0.01$ & $\mathrm{~N}$ & [31] & 9.65 & M2 \\
\hline HIP53985 & $2.89 \pm 0.28$ & $-4.37 \pm 0.04$ & $-1.28 \pm 0.04$ & $\mathrm{~N}$ & [69] & 9.57 & M2 \\
\hline HIP65859 & $2.55 \pm 0.20$ & $-4.91 \pm 0.01$ & $-1.50 \pm 0.02$ & $\mathrm{~N}$ & {$[31],[43],[6]$} & 9.05 & M2 \\
\hline HIP86287 & $2.63 \pm 0.63$ & $-5.43 \pm 0.01$ & $-1.50 \pm 0.02$ & $\mathrm{~N}$ & [25] & 9.62 & $\mathrm{M} 2$ \\
\hline HIP88574 & $2.47 \pm 0.29$ & $-5.16 \pm 0.01$ & $-1.51 \pm 0.02$ & $\mathrm{~N}$ & {$[25],[35],[6]$} & 9.37 & M2 \\
\hline HIP99701 & $<2.0$ & $-4.84 \pm 0.06$ & $-1.46 \pm 0.02$ & $\mathrm{~N}$ & {$[52],[46]$} & 7.97 & $\mathrm{M} 2$ \\
\hline HIP106440 & $2.09 \pm 0.26$ & $-5.23 \pm 0.01$ & $-1.48 \pm 0.02$ & $\mathrm{~N}$ & [35] & 8.66 & $\mathrm{M} 2$ \\
\hline HIP67155 & -- & $-5.04 \pm 0.01$ & $-1.44 \pm 0.01$ & $\mathrm{~N}$ & [43] & 8.46 & M2 \\
\hline HIP76074 & $2.35 \pm 0.23$ & $-5.21 \pm 0.01$ & $-1.44 \pm 0.02$ & $\mathrm{~N}$ & [31], [43] & 9.31 & M3 \\
\hline HIP85523 ${ }^{(\star)}$ & $2.59 \pm 0.34$ & $-5.05 \pm 0.01$ & $-1.22 \pm 0.03$ & $\mathrm{~N}$ & [31], [43] & 9.38 & M3 \\
\hline HIP94761 & $2.34 \pm 0.63$ & $-5.11 \pm 0.01$ & $-1.39 \pm 0.02$ & $\mathrm{~N}$ & {$[25],[35],[6]$} & 9.12 & M3 \\
\hline HIP116384 & $<2.0$ & $-4.60 \pm 0.01$ & $-1.33 \pm 0.02$ & $\mathrm{~N}$ & [76] & 9.59 & M3 \\
\hline HIP102409 & $9.49 \pm 0.20$ & $-4.37 \pm 0.01$ & $-0.85 \pm 0.05$ & $\mathrm{Y}$ & [35] & 8.81 & M4 \\
\hline HIP71253 & $2.83 \pm 0.51$ & $-5.51 \pm 0.01$ & $-1.43 \pm 0.04$ & $\mathrm{~N}$ & [43] & 11.32 & M4 \\
\hline HIP36208 ${ }^{(\star)}$ & $2.68 \pm 0.76$ & $-5.03 \pm 0.12$ & $-1.44 \pm 0.03$ & $\mathrm{~N}$ & {$[43],[6],[22]$} & 9.84 & M5 \\
\hline HIP1242 & $4.30 \pm 0.27$ & $-5.68 \pm 0.21$ & $-1.38 \pm 0.04$ & $\mathrm{~N}$ & [97] & 11.49 & M6 \\
\hline HIP4569 & $5.46 \pm 0.24$ & $-5.69 \pm 0.20$ & $-1.45 \pm 0.02$ & $\mathrm{~N}$ & [97] & 11.77 & M6 \\
\hline PM J07492-7642 & $7.40 \pm 0.42$ & $-4.80 \pm 0.05$ & $-0.62 \pm 0.03$ & $\mathrm{Y}+$ & [97] & 11.49 & M6 \\
\hline HIP41824 & - & $-4.87 \pm 0.09$ & $-0.83 \pm 0.01$ & $\mathrm{Y}+$ & [97] & 11.90 & M6 \\
\hline HIP48904 & - & $-4.90 \pm 0.10$ & $-0.88 \pm 0.09$ & $\mathrm{Y}$ & [97] & 11.27 & M6 \\
\hline HIP53020 & $4.92 \pm 0.22$ & $-5.53 \pm 0.10$ & $-1.43 \pm 0.10$ & $\mathrm{~N}$ & [97] & 11.64 & M6 \\
\hline HIP62452 & $4.30 \pm 0.24$ & $-5.63 \pm 0.10$ & $-1.41 \pm 0.01$ & $\mathrm{~N}$ & [97] & 11.39 & M6 \\
\hline HIP70975 & $5.81 \pm 0.23$ & $-5.70 \pm 0.23$ & $-1.44 \pm 0.04$ & $\mathrm{~N}$ & [97] & 11.92 & M6 \\
\hline PM J15012-4339 & - & $-4.89 \pm 0.10$ & $-1.05 \pm 0.09$ & $\mathrm{Y}+$ & [97] & 10.96 & M6 \\
\hline HIP75187 & $4.80 \pm 0.42$ & $-4.27 \pm 0.02$ & $-0.75 \pm 0.09$ & $\mathrm{~N}$ & [97] & 10.66 & M6 \\
\hline PM J20466-8143 & - & $-4.79 \pm 0.26$ & $-1.43 \pm 0.02$ & $\mathrm{~N}$ & [97] & 11.80 & M6 \\
\hline HIP106106 & - & $-5.48 \pm 0.06$ & $-1.43 \pm 0.06$ & $\mathrm{Y}$ & [97] & 10.33 & M6 \\
\hline HIP108706 & $39.10 \pm 1.12$ & - & $-0.61 \pm 0.07$ & - & [97] & 11.99 & M6 \\
\hline PM J22024-3704 & - & $-5.51 \pm 0.09$ & $-1.39 \pm 0.04$ & - & [97] & 11.70 & M6 \\
\hline HIP111766 & - & $-4.59 \pm 0.09$ & $-0.75 \pm 0.08$ & $\mathrm{Y}+$ & {$[96],[97],[98]$} & 11.50 & M6 \\
\hline HIP115332 & $4.94 \pm 0.23$ & $-5.13 \pm 0.20$ & $-1.37 \pm 0.05$ & $\mathrm{~N}$ & [97] & 11.70 & M6 \\
\hline
\end{tabular}


Table B.1. continued.

\begin{tabular}{cccccccc}
\hline \hline Target & $v \sin i\left(\mathrm{~km} \mathrm{~s}^{-1}\right)$ & $\log \left(R_{\mathrm{HK}}^{\prime}\right)$ & $\log I_{H \alpha_{\text {index }}}$ & Contamination & Program ID ${ }^{(2)}$ & $V$ & Spectral-type \\
\hline PM J02110-3540S & - & $-4.80 \pm 0.02$ & $-1.50 \pm 0.02$ & $\mathrm{Y}$ & {$[41],[59]$} & 11.92 & M6 \\
HIP14101 & $3.40 \pm 0.36$ & $-5.70 \pm 0.02$ & $-1.51 \pm 0.03$ & $\mathrm{~N}$ & {$[43],[31]$} & 11.38 & M6 \\
HIP22627 & $3.82 \pm 0.80$ & $-5.35 \pm 0.04$ & $-1.39 \pm 0.03$ & $\mathrm{~N}$ & {$[31],[22]$} & 11.94 & M6 \\
HIP23932 & - & - & $-1.39 \pm 0.06$ & $\mathrm{~N}$ & {$[74]$} & 10.28 & M6 \\
HIP26857 & $3.41 \pm 0.95$ & $-5.75 \pm 0.08$ & $-1.45 \pm 0.03$ & $\mathrm{~N}$ & {$[43]$} & 11.56 & M6 \\
PM J10196+1952 & $3.34 \pm 0.61$ & $-4.33 \pm 0.01$ & $-0.68 \pm 0.06$ & $\mathrm{~N}$ & {$[43]$} & 9.64 & M6 \\
PM J11319-3436 & - & $-4.12 \pm 0.09$ & $-0.71 \pm 0.02$ & $\mathrm{~N}$ & {$[14]$} & 11.72 & M6 \\
HIP67164 & $2.52 \pm 0.25$ & $-5.48 \pm 0.04$ & - & $\mathrm{N}$ & {$[31],[75]$} & 11.81 & M6 \\
HIP80824 $(\star)$ & $2.89 \pm 0.23$ & - & -- \pm & $\mathrm{N}$ & {$[73]$} & 10.10 & M6 \\
HIP87937 & - & $-5.42 \pm 0.01$ & $-1.32 \pm 0.07$ & $\mathrm{~N}$ & {$[49]$} & 9.54 & M6 \\
HIP92403 & $5.20 \pm 0.91$ & $-4.91 \pm 0.02$ & $-0.98 \pm 0.01$ & $\mathrm{~N}$ & {$[95]$} & 10.37 & M6 \\
HIP103039 & $2.70 \pm 0.66$ & $-5.17 \pm 0.01$ & $-1.37 \pm 0.01$ & $\mathrm{~N}$ & {$[6]$} & 11.41 & M6 \\
HIP113020(*) & $2.75 \pm 1.03$ & $-5.67 \pm 0.02$ & $-1.47 \pm 0.01$ & $\mathrm{~N}$ & {$[25]$} & 10.16 & M6 \\
PM J08589+0828 & - & $-4.96 \pm 0.05$ & $-0.96 \pm 0.09$ & $\mathrm{Y}$ & {$[97]$} & 11.32 & M7 \\
PM J16118-3331 & $3.80 \pm 0.35$ & $-5.39 \pm 0.03$ & $-1.50 \pm 0.05$ & $\mathrm{~N}$ & {$[97]$} & 10.98 & M7 \\
HIP102141 & - & $-3.49 \pm 0.01$ & - & $\mathrm{Y}+$ & {$[97]$} & 10.27 & M7 \\
HIP106255 & $8.47 \pm 0.37$ & $-5.28 \pm 0.07$ & $-1.22 \pm 0.01$ & - & {$[96],[97],[98]$} & 11.96 & M7 \\
HIP30920 & $5.37 \pm 0.88$ & $-5.30 \pm 0.02$ & $-0.64 \pm 0.07$ & $\mathrm{~N}$ & {$[43]$} & 11.12 & M7 \\
PM J06000+0242 & - & $-4.95 \pm 0.01$ & $-0.88 \pm 0.08$ & $\mathrm{~N}$ & {$[31],[75]$} & 11.53 & M7 \\
HIP37766 & - & $-4.26 \pm 0.01$ & $-1.32 \pm 0.01$ & $\mathrm{~N}$ & {$[62],[30]$} & 11.19 & M7 \\
HIP86214 & $3.42 \pm 0.79$ & $-4.99 \pm 0.01$ & $-1.44 \pm 0.05$ & $\mathrm{~N}$ & {$[49],[65]$} & 10.94 & M7 \\
\hline
\end{tabular}


Table B.2. Stellar parameters.

\begin{tabular}{|c|c|c|c|c|c|c|}
\hline Target & RA (hh:mm:ss) & $\operatorname{Dec}\left({ }^{\circ}:^{\prime}:^{\prime \prime}\right)$ & $T_{\text {eff }}(\mathrm{K})$ & $\log g\left(\mathrm{~cm} \mathrm{~s}^{-2}\right)$ & {$[\mathrm{Fe} / \mathrm{H}]$} & $\xi_{\mathrm{t}}\left(\mathrm{km} \mathrm{s}^{-1}\right)$ \\
\hline HIP439 & 000524.1 & -372125.3 & $3773 \pm 92$ & - & $-0.51 \pm 0.08$ & - \\
\hline HIP1242 & 001528.0 & -160801.3 & $3186 \pm 92$ & 4.681 & $-0.33 \pm 0.05$ & - \\
\hline HIP1421 & 001747.6 & +014119.3 & $5054 \pm 29$ & $2.98 \pm 0.069$ & $0.046 \pm 0.024$ & $1.396 \pm 0.029$ \\
\hline HIP1599 & 002001.9 & -645239.4 & $5962 \pm 18$ & $4.5 \pm 0.03$ & $-0.17 \pm 0.01$ & $1.08 \pm 0.03$ \\
\hline HIP2540 & 003214.7 & -630527.6 & 3924 & 4.105 & - & - \\
\hline HIP3765 & 004822.5 & +051700.2 & $4962 \pm 49$ & $4.433 \pm 0.112$ & $-0.338 \pm 0.024$ & $0.506 \pm 0.141$ \\
\hline HIP3834 & 004913.9 & -240811.5 & $5003 \pm 43$ & $3.279 \pm 0.097$ & $0.064 \pm 0.032$ & $1.098 \pm 0.05$ \\
\hline HIP4569 & 005827.8 & -275125.1 & $3402 \pm 92$ & 4.036 & $0.00 \pm 0.05$ & - \\
\hline HIP4801 & 010138.6 & -161555.3 & $4534 \pm 91$ & $2.589 \pm 0.225$ & $0.139 \pm 0.046$ & $1.355 \pm 0.091$ \\
\hline HIP6605 & 012448.7 & -025054.7 & $4922 \pm 25$ & $3.032 \pm 0.054$ & $-0.153 \pm 0.019$ & $1.188 \pm 0.025$ \\
\hline HIP6714 & 012623.6 & +200415.2 & $5040 \pm 33$ & $3.056 \pm 0.081$ & $0.049 \pm 0.027$ & $1.265 \pm 0.037$ \\
\hline HIP8102 & 014405.1 & -155622.4 & $5339 \pm 19$ & $4.45 \pm 0.06$ & $-0.51 \pm 0.01$ & $0.67 \pm 0.04$ \\
\hline HIP8768 & 015249.1 & -222605.5 & $3980 \pm 65$ & - & $0.17 \pm 0.06$ & - \\
\hline HIP9862 & 020651.9 & -190819.6 & 4700 & 2.636 & - & - \\
\hline HIP10138 & 021024.0 & -504931.1 & $5135 \pm 31$ & $4.37 \pm 0.09$ & $-0.27 \pm 0.02$ & $0.47 \pm 0.08$ \\
\hline PM J02110-3540S & 021102.4 & -354017.6 & 3017 & - & - & - \\
\hline HIP10446 & 021437.9 & +284129.1 & $4968 \pm 34$ & $3.229 \pm 0.070$ & $0.034 \pm 0.025$ & $1.089 \pm 0.037$ \\
\hline PM J02239+1812 & 022355.0 & +18 1201.9 & 3874 & - & - & - \\
\hline HIP12114 & 023603.8 & +065300.1 & 4901 & 4.626 & - & - \\
\hline HIP12754 & 024355.5 & -075444.4 & $4655 \pm 87$ & $2.811 \pm 0.216$ & $0.201 \pm 0.045$ & $1.366 \pm 0.081$ \\
\hline HIP13402 & 025231.9 & -124609.3 & $5115 \pm 44$ & $4.361 \pm 0.095$ & $1.000 \pm 0.067$ & $0.031 \pm 0.024$ \\
\hline HIP14101 & 030151.4 & -163536.0 & 3142 & 4.709 & - & - \\
\hline HIP15330 & 031744.5 & -623436.8 & $5722 \pm 21$ & $4.53 \pm 0.03$ & $-0.21 \pm 0.02$ & $0.95 \pm 0.04$ \\
\hline HIP15371 & 031811.1 & -623028.6 & $5892 \pm 16$ & $4.56 \pm 0.03$ & $-0.2 \pm 0.01$ & $1.06 \pm 0.03$ \\
\hline HIP15510 & 031953.2 & - 430417.6 & $5388 \pm 14$ & $4.379 \pm 0.036$ & $-0.385 \pm 0.011$ & $0.568 \pm 0.040$ \\
\hline HIP15514 & 031955.8 & +270416.7 & $5144 \pm 20$ & $3.255 \pm 0.08$ & $-0.15 \pm 0.016$ & $1.135 \pm 0.021$ \\
\hline PM J03219+0940 & 032157.5 & +094033.3 & $3909 \pm 92$ & - & $1.93 \pm 0.08$ & - \\
\hline HIP16537 & 033256.4 & -092729.7 & $5015 \pm 50$ & $4.405 \pm 0.095$ & $-0.134 \pm 0.027$ & $0.736 \pm 0.104$ \\
\hline PM J03340+1123 & 033400.0 & +112343.8 & $3278 \pm 92$ & - & $1.49 \pm 0.08$ & - \\
\hline HIP16641 & 033408.3 & +175000.9 & $4992 \pm 21$ & $3.528 \pm 0.041$ & $-0.3 \pm 0.015$ & $0.933 \pm 0.029$ \\
\hline HIP16711 & 033500.6 & -482511.6 & $4484 \pm 211$ & $4.279 \pm 0.821$ & $-0.162 \pm 0.124$ & $0.502 \pm 1.010$ \\
\hline HIP16852 & 033652.5 & +002410.2 & $6044 \pm 22$ & $4.162 \pm 0.03$ & $-0.009 \pm 0.017$ & $1.317 \pm 0.026$ \\
\hline PM J03377+1042 & 033744.1 & +104225.7 & 3765 & - & - & - \\
\hline HIP17027 & 033901.1 & -053732.5 & $5015 \pm 32$ & $3.611 \pm 0.081$ & $0.019 \pm 0.021$ & $0.95 \pm 0.039$ \\
\hline HIP17086 & 033938.3 & -032334.2 & $4841 \pm 48$ & $2.681 \pm 0.110$ & $0.028 \pm 0.034$ & $1.48 \pm 0.044$ \\
\hline HIP17378 & 034315.0 & -094554.7 & $5040 \pm 42$ & $3.737 \pm 0.081$ & $0.086 \pm 0.028$ & $0.955 \pm 0.059$ \\
\hline HIP17609 & 034620.1 & +261255.9 & $4007 \pm 93$ & - & $0.114 \pm 0.082$ & - \\
\hline HIP18606 & 035852.4 & -052810.3 & $4917 \pm 50$ & $3.235 \pm 0.121$ & $0.076 \pm 0.035$ & $1.173 \pm 0.061$ \\
\hline HIP19849 & 041517.6 & -073840.4 & $5084 \pm 39$ & $4.318 \pm 0.085$ & $-0.346 \pm 0.024$ & $0.400 \pm 0.121$ \\
\hline HIP20917 & 042900.1 & +215520.2 & 3973 & 4.555 & - & - \\
\hline HIP21253 & 043334.1 & -624925.1 & $4832 \pm 50$ & $3.228 \pm 0.120$ & $0.137 \pm 0.031$ & $1.102 \pm 0.056$ \\
\hline HIP22263 & 044736.2 & -165605.5 & $5844 \pm 14$ & $4.468 \pm 0.026$ & $0.029 \pm 0.011$ & $1.066 \pm 0.02$ \\
\hline HIP22319 & 044827.6 & +024254.5 & $5243 \pm 16$ & $3.677 \pm 0.023$ & $-0.117 \pm 0.013$ & $1.026 \pm 0.02$ \\
\hline HIP22449 & 044950.1 & +065740.5 & 5713 & 3.909 & - & - \\
\hline HIP22627 & 045205.7 & +062835.6 & $3099 \pm 93$ & 4.148 & - & - \\
\hline HIP23311 & 050048.7 & -054503.5 & $4726 \pm 159$ & $4.232 \pm 0.395$ & $0.218 \pm 0.086$ & $0.358 \pm 0.655$ \\
\hline HIP23452 & 050228.4 & -211523.8 & $3455 \pm 93$ & 4.351 & $-0.02 \pm 0.08$ & - \\
\hline HIP23693 & 050530.7 & -572822.8 & $6404 \pm 75$ & $4.94 \pm 0.092$ & $-0.029 \pm 0.052$ & $1.913 \pm 0.137$ \\
\hline HIP23708 & 050547.3 & -573313.7 & $3702 \pm 93$ & - & $0.14 \pm 0.08$ & - \\
\hline HIP23835 & 050726.7 & +183842.0 & $5714 \pm 17$ & $4.124 \pm 0.026$ & $-0.158 \pm 0.014$ & $1.097 \pm 0.021$ \\
\hline HIP23932 & 050835.0 & -181018.8 & 3109 & 3.718 & - & - \\
\hline HIP24130 & 051058.0 & -021513.5 & $4866 \pm 43$ & $3.289 \pm 0.098$ & $-0.008 \pm 0.028$ & $1.062 \pm 0.051$ \\
\hline HIP24186 & 051140.3 & -450103.4 & $3626 \pm 92$ & - & $-0.90 \pm 0.08$ & - \\
\hline HIP24659 & 051729.0 & -345339.8 & $4862 \pm 36$ & $3.100 \pm 0.081$ & $-0.029 \pm 0.026$ & $1.105 \pm 0.042$ \\
\hline HIP24679 & 051740.2 & -133110.9 & $4900 \pm 22$ & $3.069 \pm 0.041$ & $-0.305 \pm 0.018$ & $1.131 \pm 0.026$ \\
\hline HIP25878 & 053126.9 & -034019.7 & $3787 \pm 92$ & - & $0.19 \pm 0.08$ & - \\
\hline HIP26335 & 053631.0 & +111940.3 & $3767 \pm 92$ & 4.455 & $0.21 \pm 0.08$ & - \\
\hline HIP26366 & 053654.3 & +09 1729.1 & $4817 \pm 24$ & $2.88 \pm 0.045$ & $-0.516 \pm 0.019$ & $1.260 \pm 0.027$ \\
\hline HIP26857 & 054209.1 & +122922.4 & 3042 & - & - & - \\
\hline HIP27280 & 054652.1 & +093121.0 & $5158 \pm 28$ & $3.028 \pm 0.062$ & $-0.027 \pm 0.025$ & $1.344 \pm 0.030$ \\
\hline
\end{tabular}


Table B.2. continued.

\begin{tabular}{|c|c|c|c|c|c|c|}
\hline Target & RA (hh:mm:ss) & $\operatorname{Dec}\left({ }^{\circ}:^{\prime}:^{\prime \prime}\right)$ & $T_{\text {eff }}(\mathrm{K})$ & $\log g\left(\mathrm{~cm} \mathrm{~s}^{-2}\right)$ & {$[\mathrm{Fe} / \mathrm{H}]$} & $\xi_{\mathrm{t}}\left(\mathrm{km} \mathrm{s}^{-1}\right)$ \\
\hline PM J06000+0242 & 060003.4 & +024223.6 & 2945 & - & - & - \\
\hline HIP29271 & 061014.2 & -744509.1 & $5618 \pm 16$ & $4.413 \pm 0.033$ & $0.119 \pm 0.013$ & $0.919 \pm 0.026$ \\
\hline HIP29295 & 061034.6 & -215152.3 & $3812 \pm 92$ & - & $-0.05 \pm 0.08$ & - \\
\hline HIP30804 & 062818.8 & +101814.4 & $4603 \pm 77$ & $2.577 \pm 0.184$ & $0.156 \pm 0.043$ & $1.214 \pm 0.076$ \\
\hline HIP30815 & 062828.1 & +161418.6 & $5101 \pm 24$ & $3.261 \pm 0.053$ & $-0.031 \pm 0.02$ & $1.134 \pm 0.029$ \\
\hline HIP30920 & 062923.0 & -024844.9 & 2905 & 4.545 & - & - \\
\hline HIP31592 & 063641.0 & -191520.6 & $4785 \pm 64$ & $3.053 \pm 0.142$ & $0.205 \pm 0.037$ & $1.146 \pm 0.063$ \\
\hline HIP31634 & 063711.2 & -500217.8 & 3653 & 4.33 & - & - \\
\hline HIP31674 & 063740.8 & -125905.3 & $4915 \pm 41$ & $3.078 \pm 0.08$ & $-0.005 \pm 0.029$ & $1.261 \pm 0.041$ \\
\hline HIP32984 & 065218.4 & -051025.3 & 4757 & 4.695 & - & - \\
\hline HIP33139 & 065402.3 & -503643.7 & $5004 \pm 56$ & $3.454 \pm 0.132$ & $0.198 \pm 0.037$ & $1.243 \pm 0.063$ \\
\hline HIP33588 & 065839.8 & -554344.1 & $4576 \pm 81$ & $2.613 \pm 0.191$ & $0.116 \pm 0.040$ & $1.306 \pm 0.069$ \\
\hline HIP35846 & 072328.5 & +250302.2 & $5127 \pm 29$ & $3.20 \pm 0.072$ & $0.033 \pm 0.024$ & $1.247 \pm 0.031$ \\
\hline HIP36208 & 072724.5 & +05 1334.7 & $3277 \pm 92$ & - & $-0.03 \pm 0.08$ & - \\
\hline HIP37288 & 073923.0 & +02 1101.3 & $4014 \pm 92$ & - & $0.12 \pm 0.08$ & - \\
\hline HIP37606 & 074257.2 & -451018.4 & $5492 \pm 19$ & $3.926 \pm 0.036$ & $0.058 \pm 0.016$ & $1.075 \pm 0.024$ \\
\hline HIP37766 & 074440.2 & +033309.0 & 2945 & 4.279 & - & - \\
\hline HIP37826 & 074519.4 & +280134.7 & $4938 \pm 61$ & $3.04 \pm 0.119$ & $0.099 \pm 0.043$ & $1.447 \pm 0.06$ \\
\hline HIP37853 & 074535.2 & -341035.6 & $5758 \pm 29$ & $4.283 \pm 0.036$ & $-0.761 \pm 0.023$ & $1.035 \pm 0.059$ \\
\hline HIP38140 & 074858.9 & +04 2001.3 & $5473 \pm 21$ & $3.246 \pm 0.039$ & $-0.038 \pm 0.019$ & $1.444 \pm 0.024$ \\
\hline PM J07492-7642 & 074912.7 & -764206.5 & $2439 \pm 92$ & - & $0.00 \pm 0.08$ & - \\
\hline HIP38183 & 074928.3 & -444504.4 & $4998 \pm 35$ & $3.065 \pm 0.072$ & $-0.066 \pm 0.028$ & $1.343 \pm 0.038$ \\
\hline HIP38267 & 075023.9 & -503033.7 & $4647 \pm 38$ & $2.795 \pm 0.089$ & $-0.025 \pm 0.022$ & $1.254 \pm 0.040$ \\
\hline HIP38303 & 075051.1 & +04 2733.8 & $5048 \pm 30$ & $2.672 \pm 0.088$ & $-0.064 \pm 0.027$ & $1.425 \pm 0.03$ \\
\hline HIP38375 & 075143.0 & -211025.5 & $5055 \pm 35$ & $2.948 \pm 0.109$ & $0.1 \pm 0.029$ & $1.349 \pm 0.037$ \\
\hline HIP38748 & 075558.6 & -123630.2 & $4848 \pm 24$ & $2.912 \pm 0.046$ & $-0.284 \pm 0.018$ & $1.162 \pm 0.027$ \\
\hline HIP38908 & 075746.3 & -601812.1 & $5976 \pm 21$ & $4.54 \pm 0.03$ & $-0.29 \pm 0.02$ & $1.11 \pm 0.04$ \\
\hline HIP40239 & 081308.5 & -135501.1 & $3351 \pm 92$ & 4.116 & $0.10 \pm 0.04$ & - \\
\hline HIP40693 & 081823.8 & -123747.2 & $5423 \pm 32$ & $4.42 \pm 0.07$ & $-0.03 \pm 0.02$ & $0.77 \pm 0.06$ \\
\hline HIP41824 & 083137.6 & +192339.5 & 3001 & 4.028 & - & - \\
\hline HIP41926 & 083252.2 & -313009.7 & $5216 \pm 22$ & $4.411 \pm 0.049$ & $-0.415 \pm 0.016$ & $0.632 \pm 0.052$ \\
\hline HIP42748 & 084244.5 & +093324.4 & $4014 \pm 92$ & 4.458 & $0.09 \pm 0.04$ & - \\
\hline HIP42808 & 084318.2 & -385259.5 & $4929 \pm 54$ & $4.403 \pm 0.114$ & $-0.069 \pm 0.023$ & $0.800 \pm 0.102$ \\
\hline HIP43587 & 085236.1 & +281953.0 & $5320 \pm 50$ & $4.354 \pm 0.104$ & $0.338 \pm 0.031$ & $0.889 \pm 0.075$ \\
\hline PM J08589+0828 & 085856.4 & +082825.8 & 2906 & - & - & - \\
\hline HIP44722 & 090645.3 & -84824.7 & $3970 \pm 92$ & - & $0.17 \pm 0.08$ & - \\
\hline HIP45002 & 090957.1 & -520458.8 & $4733 \pm 34$ & $2.761 \pm 0.088$ & $-0.172 \pm 0.022$ & $1.199 \pm 0.033$ \\
\hline HIP45908 & 092137.7 & -601655.1 & $3820 \pm 92$ & - & $-0.16 \pm 0.05$ & - \\
\hline HIP46543 & 092932.4 & -041447.4 & $5160 \pm 15$ & $3.423 \pm 0.029$ & $-0.346 \pm 0.013$ & $1.1 \pm 0.019$ \\
\hline HIP48904 & 095834.3 & -462530.2 & 3095 & 3.641 & - & - \\
\hline HIP49844 & 101037.7 & -414253.8 & $4546 \pm 123$ & $2.599 \pm 0.342$ & $0.218 \pm 0.059$ & $1.497 \pm 0.110$ \\
\hline HIP49986 & 101217.7 & -34444.3 & $3606 \pm 92$ & - & $0.01 \pm 0.08$ & - \\
\hline PM J10196+1952 & 101936.3 & +195212.0 & $2991 \pm 93$ & - & $0.06 \pm 0.05$ & - \\
\hline HIP50887 & 102328.3 & -005407.8 & $5025 \pm 21$ & $3.286 \pm 0.069$ & $-0.014 \pm 0.017$ & $1.124 \pm 0.027$ \\
\hline HIP50903 & 102340.7 & -415711.6 & $5081 \pm 22$ & $3.257 \pm 0.063$ & $-0.096 \pm 0.018$ & $1.129 \pm 0.024$ \\
\hline HIP50939 & 102413.1 & +022205.1 & $4818 \pm 28$ & $2.801 \pm 0.071$ & $-0.234 \pm 0.02$ & $1.238 \pm 0.028$ \\
\hline HIP51317 & 102855.5 & +00 5027.9 & $3645 \pm 92$ & - & $-0.22 \pm 0.04$ & - \\
\hline HIP52316 & 104124.2 & -014428.3 & $5116 \pm 25$ & $3.671 \pm 0.044$ & $-0.005 \pm 0.018$ & $0.979 \pm 0.032$ \\
\hline HIP53020 & 105052.0 & +064829.7 & $3232 \pm 92$ & 4.643 & $0.08 \pm 0.04$ & - \\
\hline HIP53259 & 105332.8 & -152644.5 & 4598 & 2.628 & - & - \\
\hline HIP53985 & 110238.3 & +215801.7 & $3704 \pm 93$ & - & $0.03 \pm 0.08$ & - \\
\hline HIP54863 & 111401.8 & +08 0339.4 & $4653 \pm 67$ & $2.727 \pm 0.175$ & $0.08 \pm 0.037$ & $1.236 \pm 0.062$ \\
\hline HIP56244 & 113146.5 & -410247.2 & $3692 \pm 37$ & - & $-0.157 \pm 0.033$ & - \\
\hline PM J11319-3436 & 113155.2 & -343627.3 & 3106 & - & - & - \\
\hline HIP56452 & 113430.0 & -325000.0 & $5152 \pm 26$ & $4.47 \pm 0.08$ & $-0.42 \pm 0.02$ & $0.58 \pm 0.07$ \\
\hline HIP56830 & 113900.4 & -244313.8 & $5065 \pm 16$ & $3.333 \pm 0.03$ & $-0.746 \pm 0.014$ & $1.197 \pm 0.025$ \\
\hline HIP57079 & 114203.5 & -201738.5 & $4918 \pm 23$ & $3.109 \pm 0.068$ & $-0.117 \pm 0.017$ & $1.133 \pm 0.028$ \\
\hline HIP57137 & 114254.5 & -791822.9 & $5076 \pm 19$ & $3.324 \pm 0.063$ & $-0.108 \pm 0.015$ & $1.047 \pm 0.021$ \\
\hline HIP57443 & 114632.2 & -403004.8 & $5652 \pm 18$ & $4.4 \pm 0.05$ & $-0.3 \pm 0.01$ & $0.98 \pm 0.03$ \\
\hline HIP58576 & 120044.4 & -102641.4 & 5822 & 4.531 & - & - \\
\hline HIP62103 & 124338.0 & -013436.5 & $5086 \pm 17$ & $3.181 \pm 0.038$ & $-0.388 \pm 0.015$ & $1.191 \pm 0.022$ \\
\hline
\end{tabular}


Table B.2. continued.

\begin{tabular}{|c|c|c|c|c|c|c|}
\hline Target & RA (hh:mm:ss) & $\operatorname{Dec}\left({ }^{\circ}::^{\prime \prime}\right)$ & $T_{\text {eff }}(\mathrm{K})$ & $\log g\left(\mathrm{~cm} \mathrm{~s}^{-2}\right)$ & {$[\mathrm{Fe} / \mathrm{H}]$} & $\xi_{\mathrm{t}}\left(\mathrm{km} \mathrm{s}^{-1}\right)$ \\
\hline HIP62268 & 124537.9 & -605852.2 & $4783 \pm 55$ & $2.929 \pm 0.125$ & $0.106 \pm 0.033$ & $1.277 \pm 0.054$ \\
\hline HIP62325 & 124622.4 & +0932 26.8 & $4835 \pm 32$ & $3.159 \pm 0.079$ & $-0.037 \pm 0.023$ & $1.032 \pm 0.042$ \\
\hline HIP62452 & 124756.6 & +094505.3 & $3239 \pm 92$ & 4.26 & $0.12 \pm 0.05$ & - \\
\hline HIP62687 & 125043.6 & -004605.1 & 3818 & 4.35 & - & - \\
\hline HIP64725 & 131558.6 & -195634.2 & $4835 \pm 41$ & $3.102 \pm 0.099$ & $0.026 \pm 0.025$ & $1.117 \pm 0.041$ \\
\hline HIP64751 & 131614.3 & +190306.2 & $4917 \pm 30$ & $2.918 \pm 0.067$ & $-0.149 \pm 0.023$ & $1.286 \pm 0.028$ \\
\hline HIP64924 & 131825.0 & -181831.0 & $5585 \pm 22$ & $4.438 \pm 0.044$ & $-0.006 \pm 0.017$ & $0.852 \pm 0.039$ \\
\hline HIP65859 & 132959.7 & +102238.3 & $3750 \pm 92$ & - & $-0.19 \pm 0.04$ & - \\
\hline HIP66675 & 134007.1 & -41110.2 & $3804 \pm 92$ & 4.551 & - & - \\
\hline HIP67155 & 134542.7 & +14 5342.2 & $3526 \pm 92$ & 4.485 & - & - \\
\hline HIP67164 & 134550.7 & -175805.311 & $3131 \pm 92$ & 4.167 & - & - \\
\hline PM J13477-3225E & 134742.8 & -322551.0 & $3892 \pm 92$ & - & $0.392 \pm 0.08$ & - \\
\hline HIP67851 & 135352.3 & -351851.1 & $4867 \pm 49$ & $3.261 \pm 0.105$ & $0.020 \pm 0.031$ & $1.143 \pm 0.056$ \\
\hline HIP67890 & 135416.7 & -283409.9 & $4671 \pm 93$ & $2.983 \pm 0.212$ & $0.261 \pm 0.05$ & $1.301 \pm 0.100$ \\
\hline HIP70038 & 141953.2 & -064446.0 & $5139 \pm 32$ & $3.005 \pm 0.071$ & $0.052 \pm 0.029$ & $1.315 \pm 0.036$ \\
\hline HIP70336 & 142325.6 & -114250.0 & $4995 \pm 41$ & $2.917 \pm 0.09$ & $0.131 \pm 0.031$ & $1.362 \pm 0.0400$ \\
\hline HIP70956 & 143047.7 & -83846.7 & 3803 & 4.335 & - & - \\
\hline HIP70975 & 143101.1 & -121745.7 & $3306 \pm 92$ & 4.221 & $0.12 \pm 0.05$ & - \\
\hline HIP71253 & 143416.8 & -123110.7 & $3100 \pm 92$ & - & $0.18 \pm 0.08$ & - \\
\hline HIP73457 & 150043.4 & -110802.3 & 3906 & 4.273 & - & - \\
\hline PM J15012-4339 & 150116.5 & -433931.2 & 3138 & - & - & - \\
\hline HIP73927 & 150627.1 & -220154.1 & $4798 \pm 36$ & $2.962 \pm 0.084$ & $-0.038 \pm 0.024$ & $1.163 \pm 0.04$ \\
\hline HIP75187 & 152152.9 & +205839.8 & 3134 & 3.708 & - & - \\
\hline HIP76074 & 153213.0 & -411631.6 & $3501 \pm 92$ & - & $0.046 \pm 0.08$ & - \\
\hline HIP76219 & 153410.5 & -100350.3 & $4830 \pm 39$ & $3.138 \pm 0.103$ & $0.032 \pm 0.025$ & $1.123 \pm 0.042$ \\
\hline PM J15357-2812 & 153543.3 & -281227.2 & 3788 & - & - & - \\
\hline HIP77257 & 154626.8 & +072111.7 & $5926 \pm 14$ & $4.246 \pm 0.023$ & $0.022 \pm 0.011$ & $1.163 \pm 0.02$ \\
\hline HIP77512 & 154935.7 & +260406.8 & $5429 \pm 20$ & $3.422 \pm 0.039$ & $-0.089 \pm 0.018$ & $1.354 \pm 0.023$ \\
\hline PM J15543-3536 & 155421.4 & -353616.8 & 3831 & 53 & - & - \\
\hline PM J16015-4710 & 160131.0 & -471032.8 & 3795 & - & - & - \\
\hline HIP78508 & 160138.4 & -440927.0 & $3746 \pm 92$ & 2.39 & - & - \\
\hline PM J16046-5521 & 160440.7 & $\begin{array}{lll}-5521 & 12.2\end{array}$ & $3110 \pm 92$ & - & - & - \\
\hline PM J16051-0508 & 160509.0 & -50858.7 & 3743 & - & - & - \\
\hline HIP79137 & 160911.0 & +06 22 49.8 & $4931 \pm 49$ & $3.592 \pm 0.109$ & $0.127 \pm 0.03$ & $0.948 \pm 0.062$ \\
\hline HIP79148 & 160915.9 & -504205.1 & 3795 & 1.764 & - & - \\
\hline PM J16118-3331 & 161148.4 & -333119.8 & 2954 & - & - & - \\
\hline HIP79522 & 161338.6 & -012830.5 & $4901 \pm 27$ & $2.863 \pm 0.06$ & $-0.159 \pm 0.022$ & $1.281 \pm 0.028$ \\
\hline HIP79605 & 161439.3 & -183207.2 & $4764 \pm 46$ & $2.849 \pm 0.118$ & $0.086 \pm 0.029$ & $1.263 \pm 0.08$ \\
\hline HIP80824 & 163018.0 & -123944.7 & 3089 & 4.314 & - & - \\
\hline HIP80910 & 163122.9 & -263215.2 & $4738 \pm 66$ & $2.943 \pm 0.15$ & $0.169 \pm 0.037$ & $1.235 \pm 0.063$ \\
\hline HIP81300 & 163621.1 & -021925.8 & $5274 \pm 40$ & $4.51 \pm 0.08$ & - & $0.9 \pm 0.08$ \\
\hline HIP81705 & 164122.2 & -132343.1 & $3540 \pm 92$ & - & $1.68 \pm 0.08$ & - \\
\hline PM J16565-4749 & 165635.9 & -474951.2 & 3804 & - & - & - \\
\hline HIP82989 & 165732.1 & +135302.2 & $4986 \pm 33$ & $3.324 \pm 0.075$ & $0.047 \pm 0.023$ & $1.069 \pm 0.037$ \\
\hline HIP83541 & 170427.8 & -283455.3 & $5419 \pm 42$ & $4.364 \pm 0.066$ & $0.318 \pm 0.03$ & $0.878 \pm 0.068$ \\
\hline HIP83688 & 170613.0 & +102713.9 & $5218 \pm 25$ & $3.25 \pm 0.039$ & $0.019 \pm 0.022$ & $1.317 \pm 0.026$ \\
\hline HIP84478 & 171613.7 & -263236.3 & $4561 \pm 84$ & $4.24 \pm 0.26$ & $-0.34 \pm 0.04$ & $0.47 \pm 0.37$ \\
\hline PM J17173+0003 & 171718.2 & +000343.8 & $3209 \pm 92$ & - & $0.26 \pm 0.08$ & - \\
\hline HIP85207 & 172442.0 & -212629.1 & $5101 \pm 34$ & $2.913 \pm 0.092$ & $0.109 \pm 0.03$ & $1.363 \pm 0.036$ \\
\hline HIP85523 & 172839.9 & -465342.2 & $3456 \pm 92$ & - & $-0.257 \pm 0.08$ & - \\
\hline HIP85647 & 173011.2 & -513813.0 & $4293 \pm 93$ & - & $0.261 \pm 0.082$ & - \\
\hline HIP85665 & 173022.7 & +053254.8 & $3791 \pm 65$ & - & $-0.147 \pm 0.058$ & - \\
\hline HIP86214 & 173703.6 & -441908.7 & $2992 \pm 92$ & 4.348 & - & - \\
\hline HIP86287 & 173753.3 & +183529.7 & $3728 \pm 65$ & - & $-0.362 \pm 0.058$ & - \\
\hline HIP86400 & 173917.0 & +033319.7 & $4871 \pm 95$ & $4.395 \pm 0.216$ & $-0.07 \pm 0.041$ & $0.445 \pm 0.276$ \\
\hline HIP86796 & 174408.7 & -515000.9 & $5802 \pm 22$ & $4.271 \pm 0.034$ & $0.311 \pm 0.017$ & $1.115 \pm 0.027$ \\
\hline HIP87540 & 175303.5 & -105358.2 & $4696 \pm 47$ & $2.576 \pm 0.114$ & $-0.081 \pm 0.029$ & $1.356 \pm 0.042$ \\
\hline HIP87834 & 175637.8 & +183644.7 & $4864 \pm 56$ & $3.47 \pm 0.124$ & $0.166 \pm 0.031$ & $0.967 \pm 0.065$ \\
\hline HIP87937 & 175748.5 & +044131.0 & $3092 \pm 92$ & 4.831 & - & - \\
\hline HIP88574 & 180507.5 & -30152.5 & $3743 \pm 46$ & - & $-0.284 \pm 0.041$ & - \\
\hline HIP89962 & 182118.9 & -025349.6 & $4962 \pm 19$ & $3.126 \pm 0.044$ & $-0.161 \pm 0.015$ & $1.188 \pm 0.021$ \\
\hline
\end{tabular}


Table B.2. continued.

\begin{tabular}{|c|c|c|c|c|c|c|}
\hline Target & RA (hh:mm:ss) & $\operatorname{Dec}\left({ }^{\circ}:^{\prime}:^{\prime \prime}\right)$ & $T_{\text {eff }}(\mathrm{K})$ & $\log g\left(\mathrm{~cm} \mathrm{~s}^{-2}\right)$ & {$[\mathrm{Fe} / \mathrm{H}]$} & $\xi_{\mathrm{t}}\left(\mathrm{km} \mathrm{s}^{-1}\right)$ \\
\hline PM J18400-1644 & 184000.6 & -164426.8 & 3758 & - & - & - \\
\hline HIP92403 & 184949.3 & -235010.3 & $3099 \pm 92$ & 4.74 & - & - \\
\hline HIP92444 & 185021.2 & -265524.9 & $4329 \pm 113$ & $4.09 \pm 0.41$ & $-0.54 \pm 0.03$ & $0.14 \pm 0.1$ \\
\hline PM J18548+1058 & 185453.6 & +105840.303 & $4051 \pm 66$ & - & $0.40 \pm 0.06$ & - \\
\hline HIP93069 & 185730.6 & -555930.5 & $4293 \pm 92$ & 4.19 & $0.43 \pm 0.08$ & - \\
\hline HIP93101 & 185800.1 & +05 5429.8 & $3932 \pm 92$ & - & $0.05 \pm 0.08$ & - \\
\hline HIP93203 & 185905.7 & +133721.2 & 6087 & 3.374 & - & - \\
\hline HIP94521 & 191416.0 & -084308.5 & $4890 \pm 37$ & $3.163 \pm 0.096$ & $-0.055 \pm 0.025$ & $1.137 \pm 0.039$ \\
\hline HIP94739 & 191642.9 & -455321.4 & 3673 & 4.212 & - & - \\
\hline HIP94761 & 191655.3 & +051008.7 & $3570 \pm 92$ & - & $0.04 \pm 0.08$ & - \\
\hline HIP95447 & 192457.8 & +115634.3 & $5676 \pm 45$ & $4.21 \pm 0.079$ & $0.408 \pm 0.035$ & $1.151 \pm 0.056$ \\
\hline HIP96229 & 193405.2 & +072245.5 & $4623 \pm 89$ & $2.824 \pm 0.214$ & $0.135 \pm 0.046$ & $1.287 \pm 0.084$ \\
\hline PM J19473-2424 & 194720.3 & -242439.4 & 3780 & - & - & - \\
\hline HIP98066 & 195550.2 & -261758.9 & $5535 \pm 20$ & $3.828 \pm 0.032$ & $0.033 \pm 0.017$ & $1.164 \pm 0.024$ \\
\hline HIP98767 & 200336.9 & +295353.1 & $5672 \pm 32$ & $4.411 \pm 0.045$ & $0.274 \pm 0.024$ & $0.946 \pm 0.08$ \\
\hline HIP98920 & 200509.5 & +195927.2 & $4781 \pm 54$ & $2.933 \pm 0.123$ & $0.052 \pm 0.033$ & $1.317 \pm 0.052$ \\
\hline HIP99171 & 200801.8 & -004040.9 & $4792 \pm 43$ & $3.135 \pm 0.103$ & $-0.012 \pm 0.025$ & $1.058 \pm 0.045$ \\
\hline HIP99240 & 200841.9 & -661045.6 & $5648 \pm 28$ & $4.34 \pm 0.06$ & $0.99 \pm 0.04$ & $0.38 \pm 0.02$ \\
\hline HIP99461 & 201111.6 & -360550.6 & $4922 \pm 88$ & $4.349 \pm 0.153$ & $-0.574 \pm 0.063$ & $0.018 \pm 1.760$ \\
\hline HIP99701 & 201353.4 & -450950.4 & $3754 \pm 92$ & - & $-0.07 \pm 0.06$ & - \\
\hline HIP99825 & 201516.6 & -270157.1 & $5078 \pm 61$ & $4.427 \pm 0.125$ & $-0.019 \pm 0.032$ & $0.645 \pm 0.137$ \\
\hline HIP99894 & 201606.0 & +043451.3 & $5162 \pm 28$ & $3.432 \pm 0.045$ & $-0.162 \pm 0.024$ & $1.104 \pm 0.037$ \\
\hline HIP99913 & 201619.8 & +213555.5 & $4841 \pm 50$ & $2.934 \pm 0.117$ & $0.129 \pm 0.032$ & $1.31 \pm 0.048$ \\
\hline HIP101221 & 203104.3 & -150321.8 & $4749 \pm 141$ & $2.66 \pm 0.297$ & $-0.148 \pm 0.138$ & $0.05 \pm 0.289$ \\
\hline HIP101345 & 203223.5 & -095113.1 & $5683 \pm 13$ & $4.056 \pm 0.02$ & $0.077 \pm 0.011$ & $1.125 \pm 0.015$ \\
\hline HIP101916 & 203907.6 & +100510.1 & $5722 \pm 16$ & $3.835 \pm 0.029$ & $0.071 \pm 0.014$ & $1.294 \pm 0.02$ \\
\hline HIP102141 & 204151.1 & -322606.6 & 2992 & 3.467 & - & - \\
\hline HIP102409 & 204509.5 & -312027.0 & 3399 & 3.84 & - & - \\
\hline PM J20466-8143 & 204637.1 & -814313.6 & $3561 \pm 92$ & - & $-0.06 \pm 0.05$ & - \\
\hline HIP102891 & 205041.7 & -123241.0 & $4663 \pm 42$ & $2.581 \pm 0.093$ & $-0.186 \pm 0.026$ & $1.308 \pm 0.038$ \\
\hline HIP103039 & 205233.0 & -165829.1 & 3029 & 4.584 & - & - \\
\hline HIP106106 & 212936.8 & +173835.6 & 3142 & 4.037 & - & - \\
\hline HIP106255 & 213118.6 & -94726.3 & $3127 \pm 92$ & 4.311 & $0.15 \pm 0.05$ & - \\
\hline HIP106440 & 213333.9 & -490032.0 & $3664 \pm 92$ & - & $-0.189 \pm 0.08$ & - \\
\hline HIP107649 & 214815.6 & -471810.4 & $5924 \pm 15$ & $4.454 \pm 0.023$ & $0.013 \pm 0.012$ & $1.025 \pm 0.023$ \\
\hline HIP107705 & 214905.8 & -720608.8 & $3228 \pm 92$ & - & $0.24 \pm 0.05$ & - \\
\hline HIP108513 & 215859.6 & +190113.4 & $4920 \pm 56$ & $3.206 \pm 0.123$ & $0.176 \pm 0.036$ & $1.201 \pm 0.056$ \\
\hline HIP108706 & 220113.1 & +281824.9 & 3029 & 4.39 & - & - \\
\hline HIP108782 & 220210.3 & +012400.9 & $3848 \pm 92$ & - & $0.002 \pm 0.08$ & - \\
\hline PM J22024-3704 & 220229.4 & -370451.3 & $3434 \pm 92$ & - & $-0.05 \pm 0.04$ & - \\
\hline HIP108868 & 220316.4 & -063120.7 & $5045 \pm 40$ & $2.918 \pm 0.09$ & $0.075 \pm 0.033$ & $1.43 \pm 0.042$ \\
\hline HIP108870 & 220317.4 & -564647.3 & $4616 \pm 159$ & $4.248 \pm 0.382$ & $-0.162 \pm 0.102$ & $0.234 \pm 1.545$ \\
\hline HIP109577 & 221151.3 & +1602 26.1 & $5010 \pm 33$ & $3.307 \pm 0.074$ & $0.083 \pm 0.027$ & $1.085 \pm 0.044$ \\
\hline HIP109822 & 221438.2 & -154903.6 & $4980 \pm 26$ & $3.673 \pm 0.046$ & $-0.253 \pm 0.017$ & $0.84 \pm 0.036$ \\
\hline HIP110109 & 221815.1 & -533731.9 & $5872 \pm 18$ & $4.47 \pm 0.04$ & $-0.19 \pm 0.01$ & $1.06 \pm 0.03$ \\
\hline HIP111766 & 223829.7 & -652242.5 & 3075 & 3.783 & - & - \\
\hline HIP113020 & 225316.7 & -141548.9 & $3049 \pm 92$ & 4.23 & - & - \\
\hline HIP113148 & 225445.6 & -161618.3 & $4684 \pm 79$ & $3.038 \pm 0.18$ & $0.189 \pm 0.043$ & $1.211 \pm 0.084$ \\
\hline HIP113283 & 225623.8 & -313354.6 & $4575 \pm 72$ & $4.239 \pm 0.244$ & $-0.089 \pm 0.032$ & $0.600 \pm 0.263$ \\
\hline HIP113296 & 225634.8 & +163312.5 & $3769 \pm 92$ & - & $0.026 \pm 0.08$ & - \\
\hline HIP113357 & 225727.9 & +204607.3 & $5805 \pm 16$ & $4.347 \pm 0.022$ & $0.215 \pm 0.013$ & $1.035 \pm 0.021$ \\
\hline HIP113576 & 230016.7 & -223128.2 & $3766 \pm 92$ & - & $0.25 \pm 0.08$ & - \\
\hline HIP113801 & 230244.3 & -205213.4 & $4941 \pm 25$ & $3.073 \pm 0.064$ & $-0.117 \pm 0.019$ & $1.219 \pm 0.027$ \\
\hline HIP114526 & 231149.3 & +265051.3 & $4951 \pm 29$ & $3.094 \pm 0.072$ & $-0.074 \pm 0.022$ & $1.138 \pm 0.031$ \\
\hline HIP114699 & 231406.0 & -624156.3 & $5356 \pm 16$ & $3.921 \pm 0.029$ & $-0.117 \pm 0.013$ & $0.949 \pm 0.021$ \\
\hline HIP115332 & 232137.4 & +17 1726.0 & $3156 \pm 92$ & 4.124 & $0.28 \pm 0.04$ & - \\
\hline HIP115623 & 232522.7 & +232414.4 & 6012 & 2.918 & - & - \\
\hline HIP115953 & 232932.0 & -043155.9 & $4722 \pm 113$ & $3.257 \pm 0.26$ & $0.364 \pm 0.063$ & $1.135 \pm 0.124$ \\
\hline HIP116384 & 233500.2 & +013619.4 & $3635 \pm 92$ & - & $0.54 \pm 0.08$ & - \\
\hline HIP116422 & 233528.6 & +011847.5 & $4646 \pm 62$ & $2.65 \pm 0.142$ & $0.082 \pm 0.035$ & $1.303 \pm 0.06$ \\
\hline HIP116624 & 233802.9 & -150541.4 & $4731 \pm 40$ & $2.458 \pm 0.11$ & $-0.064 \pm 0.028$ & $1.453 \pm 0.04$ \\
\hline HIP117473 & 234912.5 & +02 2404.9 & $3725 \pm 92$ & - & $-0.513 \pm 0.08$ & - \\
\hline
\end{tabular}


S. Hojjatpanah et al.: Catalog for the ESPRESSO blind radial velocity exoplanet survey

Table B.3. Chemical abundances relative to the Sun.

\begin{tabular}{|c|c|c|c|c|c|c|c|c|c|c|}
\hline Star & {$[\mathrm{Na} / \mathrm{H}]$} & {$[\mathrm{MgI} / \mathrm{H}]$} & {$[\mathrm{AlI} / \mathrm{H}]$} & {$[\mathrm{SiI} / \mathrm{H}]$} & {$[\mathrm{CaI} / \mathrm{H}]$} & {$[\mathrm{ScII} / \mathrm{H}]$} & {$[\mathrm{TiI} / \mathrm{H}]$} & {$[\mathrm{TiII} / \mathrm{H}]$} & {$[\mathrm{CrI} / \mathrm{H}]$} & {$[\mathrm{NiI} / \mathrm{H}]$} \\
\hline HIP1421 & $0.150 \pm 0.017$ & $0.107 \pm 0.056$ & $0.066 \pm 0.022$ & $0.119 \pm 0.063$ & $0.031 \pm 0.056$ & $0.057 \pm 0.021$ & $0.032 \pm 0.070$ & $0.016 \pm 0.056$ & $0.013 \pm 0.042$ & $0.007 \pm 0.044$ \\
\hline HIP1599 & $-0.250 \pm 0.021$ & $-0.146 \pm 0.045$ & $-0.192 \pm 0.031$ & $-0.180 \pm 0.025$ & $-0.208 \pm 0.063$ & $-0.137 \pm 0.024$ & $-0.155 \pm 0.030$ & $-0.166 \pm 0.015$ & $-0.230 \pm 0.034$ & $-0.233 \pm 0.014$ \\
\hline HIP3765 & $-0.347 \pm 0.013$ & $-0.183 \pm 0.081$ & $-0.217 \pm 0.017$ & $-0.261 \pm 0.071$ & $-0.170 \pm 0.147$ & $-0.309 \pm 0.059$ & $-0.149 \pm 0.074$ & $-0.385 \pm 0.029$ & $-0.282 \pm 0.073$ & $-0.352 \pm 0.038$ \\
\hline HIP3834 & $0.015 \pm 0.040$ & $0.117 \pm 0.073$ & $0.105 \pm 0.026$ & $0.104 \pm 0.067$ & $0.078 \pm 0.153$ & $0.064 \pm 0.026$ & $0.100 \pm 0.097$ & $0.025 \pm 0.042$ & $0.058 \pm 0.070$ & $0.044 \pm 0.053$ \\
\hline HIP4801 & $0.359 \pm 0.052$ & $0.310 \pm 0.098$ & $0.404 \pm 0.035$ & $0.316 \pm 0.157$ & $0.081 \pm 0.066$ & $0.178 \pm 0.083$ & $0.172 \pm 0.136$ & $0.042 \pm 0.113$ & $0.090 \pm 0.061$ & $0.199 \pm 0.101$ \\
\hline HIP6605 & $-0.169 \pm 0.045$ & $0.016 \pm 0.044$ & $-0.038 \pm 0.021$ & $-0.090 \pm 0.062$ & $-0.103 \pm 0.053$ & $-0.114 \pm 0.030$ & $-0.084 \pm 0.079$ & $-0.134 \pm 0.039$ & $-0.189 \pm 0.029$ & $-0.178 \pm 0.037$ \\
\hline HIP6714 & $0.011 \pm 0.021$ & $-0.008 \pm 0.080$ & $0.082 \pm 0.052$ & $0.082 \pm 0.067$ & $0.062 \pm 0.039$ & $0.005 \pm 0.023$ & $0.069 \pm 0.121$ & $-0.058 \pm 0.069$ & $0.045 \pm 0.037$ & $0.001 \pm 0.040$ \\
\hline HIP8102 & $-0.533 \pm 0.028$ & $-0.257 \pm 0.072$ & $-0.296 \pm 0.027$ & $-0.407 \pm 0.020$ & $-0.364 \pm 0.095$ & $-0.405 \pm 0.046$ & $-0.265 \pm 0.036$ & $-0.397 \pm 0.031$ & $-0.495 \pm 0.044$ & $-0.538 \pm 0.023$ \\
\hline HIP10138 & $-0.322 \pm 0.035$ & $-0.110 \pm 0.053$ & $-0.131 \pm 0.033$ & $-0.224 \pm 0.040$ & $-0.237 \pm 0.077$ & $-0.310 \pm 0.021$ & $-0.115 \pm 0.032$ & $-0.294 \pm 0.017$ & $-0.247 \pm 0.056$ & $-0.310 \pm 0.020$ \\
\hline HIP10446 & $0.012 \pm 0.020$ & $0.029 \pm 0.071$ & $0.091 \pm 0.023$ & $0.063 \pm 0.071$ & $0.039 \pm 0.064$ & $0.031 \pm 0.018$ & $0.062 \pm 0.074$ & $0.001 \pm 0.043$ & $0.010 \pm 0.064$ & $0.021 \pm 0.045$ \\
\hline HIP12754 & $0.372 \pm 0.080$ & $0.356 \pm 0.071$ & $0.466 \pm 0.035$ & $0.342 \pm 0.147$ & $0.159 \pm 0.069$ & $0.348 \pm 0.116$ & $0.244 \pm 0.103$ & $0.068 \pm 0.092$ & $0.175 \pm 0.059$ & $0.275 \pm 0.111$ \\
\hline HIP13402 & $-0.064 \pm 0.034$ & $-0.064 \pm 0.027$ & $0.125 \pm 0.122$ & $0.048 \pm 0.112$ & $0.092 \pm 0.079$ & $-0.088 \pm 0.041$ & $0.059 \pm 0.041$ & $-0.078 \pm 0.067$ & $0.060 \pm 0.040$ & $0.008 \pm 0.034$ \\
\hline HIP15330 & $-0.287 \pm 0.027$ & $-0.154 \pm 0.069$ & $-0.163 \pm 0.038$ & $-0.204 \pm 0.030$ & $-0.204 \pm 0.085$ & $-0.179 \pm 0.034$ & $-0.153 \pm 0.033$ & $-0.198 \pm 0.018$ & $-0.208 \pm 0.023$ & $-0.246 \pm 0.014$ \\
\hline HIP15371 & $-0.290 \pm 0.031$ & $-0.159 \pm 0.061$ & $-0.197 \pm 0.048$ & $-0.215 \pm 0.010$ & $-0.207 \pm 0.052$ & $-0.182 \pm 0.026$ & $-0.178 \pm 0.019$ & $-0.197 \pm 0.016$ & $-0.237 \pm 0.037$ & $-0.245 \pm 0.014$ \\
\hline HIP 15510 & $-0.332 \pm 0.016$ & $-0.114 \pm 0.046$ & $-0.086 \pm 0.018$ & $-0.221 \pm 0.028$ & $-0.160 \pm 0.093$ & $-0.201 \pm 0.024$ & $-0.100 \pm 0.038$ & $-0.158 \pm 0.030$ & $-0.355 \pm 0.039$ & $-0.363 \pm 0.030$ \\
\hline HIP 15514 & $-0.256 \pm 0.050$ & $-0.114 \pm 0.038$ & $-0.105 \pm 0.026$ & $-0.134 \pm 0.031$ & $-0.097 \pm 0.062$ & $-0.163 \pm 0.029$ & $-0.100 \pm 0.049$ & $-0.131 \pm 0.024$ & $-0.163 \pm 0.034$ & $-0.222 \pm 0.028$ \\
\hline HIP16537 & $-0.245 \pm 0.021$ & $-0.212 \pm 0.024$ & $-0.155 \pm 0.026$ & $-0.092 \pm 0.075$ & $-0.062 \pm 0.107$ & $-0.189 \pm 0.043$ & $-0.089 \pm 0.046$ & $-0.186 \pm 0.064$ & $-0.100 \pm 0.091$ & $-0.179 \pm 0.046$ \\
\hline HIP16641 & $-0.328 \pm 0.051$ & $-0.101 \pm 0.036$ & $-0.059 \pm 0.018$ & $-0.158 \pm 0.033$ & $-0.178 \pm 0.073$ & $-0.121 \pm 0.024$ & $-0.077 \pm 0.062$ & $-0.112 \pm 0.019$ & $-0.305 \pm 0.066$ & $-0.293 \pm 0.031$ \\
\hline HIP16852 & $-0.020 \pm 0.026$ & $0.094 \pm 0.033$ & $-0.061 \pm 0.024$ & $-0.018 \pm 0.070$ & $-0.033 \pm 0.064$ & $0.010 \pm 0.072$ & $-0.027 \pm 0.026$ & $-0.038 \pm 0.014$ & $-0.069 \pm 0.033$ & $-0.051 \pm 0.021$ \\
\hline HIP17027 & $0.105 \pm 0.089$ & $0.106 \pm 0.037$ & $0.088 \pm 0.030$ & $0.078 \pm 0.051$ & $0.017 \pm 0.060$ & $0.069 \pm 0.024$ & $0.050 \pm 0.051$ & $0.051 \pm 0.055$ & $0.007 \pm 0.056$ & $0.032 \pm 0.048$ \\
\hline HIP17086 & $-0.019 \pm 0.086$ & $0.029 \pm 0.070$ & $0.119 \pm 0.022$ & $0.112 \pm 0.077$ & $-0.039 \pm 0.076$ & $0.051 \pm 0.038$ & $0.040 \pm 0.089$ & $-0.023 \pm 0.068$ & $-0.019 \pm 0.035$ & $0.004 \pm 0.057$ \\
\hline HIP17378 & $0.136 \pm 0.016$ & $0.181 \pm 0.033$ & $0.158 \pm 0.023$ & $0.184 \pm 0.072$ & $0.048 \pm 0.053$ & $0.136 \pm 0.043$ & $0.141 \pm 0.048$ & $0.080 \pm 0.038$ & $0.069 \pm 0.060$ & $0.117 \pm 0.041$ \\
\hline HIP18606 & $0.201 \pm 0.069$ & $0.111 \pm 0.017$ & $0.186 \pm 0.018$ & $0.146 \pm 0.083$ & $0.069 \pm 0.072$ & $0.088 \pm 0.041$ & $0.131 \pm 0.102$ & $0.006 \pm 0.065$ & $0.070 \pm 0.094$ & $0.097 \pm 0.052$ \\
\hline HIP19849 & $-0.308 \pm 0.031$ & $-0.125 \pm 0.055$ & $-0.051 \pm 0.027$ & $-0.189 \pm 0.031$ & $-0.137 \pm 0.104$ & $-0.244 \pm 0.037$ & $-0.018 \pm 0.060$ & $-0.209 \pm 0.031$ & $-0.278 \pm 0.069$ & $-0.310 \pm 0.033$ \\
\hline HIP21253 & $0.149 \pm 0.044$ & $0.167 \pm 0.045$ & $0.265 \pm 0.038$ & $0.285 \pm 0.090$ & $0.025 \pm 0.055$ & $0.193 \pm 0.035$ & $0.148 \pm 0.074$ & $0.118 \pm 0.059$ & $0.089 \pm 0.043$ & $0.176 \pm 0.070$ \\
\hline HIP22263 & $-0.131 \pm 0.024$ & $-0.031 \pm 0.020$ & $-0.058 \pm 0.034$ & $-0.026 \pm 0.049$ & $0.025 \pm 0.074$ & $-0.069 \pm 0.040$ & $0.024 \pm 0.029$ & $-0.027 \pm 0.016$ & $0.025 \pm 0.018$ & $-0.044 \pm 0.014$ \\
\hline HIP22319 & $-0.198 \pm 0.018$ & $0.001 \pm 0.031$ & $-0.080 \pm 0.025$ & $-0.112 \pm 0.025$ & $-0.110 \pm 0.047$ & $-0.116 \pm 0.026$ & $-0.058 \pm 0.034$ & $-0.083 \pm 0.021$ & $-0.120 \pm 0.031$ & $-0.163 \pm 0.021$ \\
\hline HIP23311 & $0.304 \pm 0.073$ & $0.285 \pm 0.112$ & $0.400 \pm 0.035$ & $0.238 \pm 0.060$ & $0.075 \pm 0.080$ & $0.268 \pm 0.072$ & $0.399 \pm 0.055$ & $0.135 \pm 0.131$ & $0.235 \pm 0.069$ & $0.278 \pm 0.062$ \\
\hline HIP23835 & $-0.195 \pm 0.018$ & $-0.041 \pm 0.023$ & $-0.044 \pm 0.024$ & $-0.098 \pm 0.034$ & $-0.097 \pm 0.069$ & $-0.045 \pm 0.039$ & $-0.060 \pm 0.042$ & $-0.053 \pm 0.016$ & $-0.201 \pm 0.024$ & $-0.196 \pm 0.022$ \\
\hline HIP24130 & $0.000 \pm 0.020$ & $0.154 \pm 0.027$ & $0.168 \pm 0.021$ & $0.147 \pm 0.087$ & $0.026 \pm 0.072$ & $0.037 \pm 0.026$ & $0.101 \pm 0.093$ & $0.037 \pm 0.039$ & $-0.030 \pm 0.035$ & $-0.014 \pm 0.050$ \\
\hline HIP24659 & $-0.055 \pm 0.034$ & $0.129 \pm 0.047$ & $0.102 \pm 0.035$ & $0.056 \pm 0.094$ & $-0.059 \pm 0.057$ & $0.028 \pm 0.014$ & $0.026 \pm 0.061$ & $-0.005 \pm 0.053$ & $-0.064 \pm 0.031$ & $-0.016 \pm 0.041$ \\
\hline HIP24679 & $-0.387 \pm 0.053$ & $-0.116 \pm 0.036$ & $-0.115 \pm 0.041$ & $-0.196 \pm 0.039$ & $-0.215 \pm 0.054$ & $-0.193 \pm 0.011$ & $-0.162 \pm 0.086$ & $-0.186 \pm 0.037$ & $-0.325 \pm 0.069$ & $-0.327 \pm 0.031$ \\
\hline HIP26366 & $-0.425 \pm 0.040$ & $-0.227 \pm 0.021$ & $-0.204 \pm 0.024$ & $-0.320 \pm 0.032$ & $-0.354 \pm 0.050$ & $-0.323 \pm 0.022$ & $-0.271 \pm 0.063$ & $-0.262 \pm 0.029$ & $-0.542 \pm 0.045$ & $-0.522 \pm 0.035$ \\
\hline HIP27280 & $0.032 \pm 0.014$ & $-0.055 \pm 0.075$ & $-0.037 \pm 0.016$ & $-0.011 \pm 0.048$ & $0.006 \pm 0.051$ & $-0.036 \pm 0.024$ & $-0.018 \pm 0.062$ & $-0.061 \pm 0.056$ & $-0.056 \pm 0.033$ & $-0.106 \pm 0.039$ \\
\hline HIP29271 & $0.103 \pm 0.014$ & $0.131 \pm 0.027$ & $0.153 \pm 0.017$ & $0.103 \pm 0.058$ & $0.047 \pm 0.051$ & $0.121 \pm 0.059$ & $0.151 \pm 0.024$ & $0.109 \pm 0.020$ & $0.126 \pm 0.030$ & $0.140 \pm 0.021$ \\
\hline HIP30804 & $0.299 \pm 0.058$ & $0.386 \pm 0.138$ & $0.300 \pm 0.058$ & $0.246 \pm 0.167$ & $0.089 \pm 0.080$ & $0.194 \pm 0.058$ & $0.163 \pm 0.123$ & $0.058 \pm 0.103$ & $0.115 \pm 0.107$ & $0.196 \pm 0.094$ \\
\hline HIP30815 & $0.127 \pm 0.020$ & $0.113 \pm 0.047$ & $0.018 \pm 0.023$ & $0.046 \pm 0.057$ & $0.029 \pm 0.069$ & $-0.005 \pm 0.020$ & $0.009 \pm 0.059$ & $-0.026 \pm 0.040$ & $-0.047 \pm 0.037$ & $-0.049 \pm 0.032$ \\
\hline HIP31592 & $0.497 \pm 0.116$ & $0.354 \pm 0.069$ & $0.331 \pm 0.040$ & $0.285 \pm 0.122$ & $0.146 \pm 0.069$ & $0.262 \pm 0.081$ & $0.234 \pm 0.083$ & $0.076 \pm 0.071$ & $0.168 \pm 0.078$ & $0.233 \pm 0.064$ \\
\hline HIP31674 & $-0.058 \pm 0.062$ & $0.010 \pm 0.049$ & $0.038 \pm 0.025$ & $0.068 \pm 0.087$ & $-0.013 \pm 0.067$ & $0.021 \pm 0.019$ & $0.023 \pm 0.076$ & $0.009 \pm 0.056$ & $-0.045 \pm 0.048$ & $-0.041 \pm 0.042$ \\
\hline HIP33139 & $0.372 \pm 0.025$ & $0.199 \pm 0.043$ & $0.301 \pm 0.027$ & $0.338 \pm 0.094$ & $0.089 \pm 0.062$ & $0.326 \pm 0.060$ & $0.167 \pm 0.061$ & $0.145 \pm 0.054$ & $0.178 \pm 0.060$ & $0.234 \pm 0.074$ \\
\hline HIP33588 & $0.400 \pm 0.133$ & $0.311 \pm 0.076$ & $0.353 \pm 0.045$ & $0.256 \pm 0.098$ & $0.019 \pm 0.087$ & $0.105 \pm 0.070$ & $0.113 \pm 0.109$ & $0.015 \pm 0.088$ & $0.089 \pm 0.084$ & $0.183 \pm 0.080$ \\
\hline HIP35846 & $-0.103 \pm 0.019$ & $-0.022 \pm 0.065$ & $-0.042 \pm 0.022$ & $0.017 \pm 0.040$ & $0.008 \pm 0.049$ & $0.025 \pm 0.017$ & $0.019 \pm 0.062$ & $-0.023 \pm 0.061$ & $-0.001 \pm 0.035$ & $-0.041 \pm 0.039$ \\
\hline HIP37606 & $0.005 \pm 0.026$ & $0.081 \pm 0.031$ & $0.081 \pm 0.031$ & $0.039 \pm 0.032$ & $-0.007 \pm 0.059$ & $0.116 \pm 0.023$ & $0.085 \pm 0.022$ & $0.086 \pm 0.043$ & $0.033 \pm 0.033$ & $0.053 \pm 0.027$ \\
\hline HIP37826 & $0.132 \pm 0.023$ & $0.127 \pm 0.130$ & $0.165 \pm 0.055$ & $0.163 \pm 0.107$ & $0.011 \pm 0.068$ & $0.054 \pm 0.039$ & $0.088 \pm 0.079$ & $0.007 \pm 0.110$ & $0.032 \pm 0.077$ & $0.087 \pm 0.102$ \\
\hline HIP37853 & $-0.670 \pm 0.059$ & $-0.439 \pm 0.059$ & $-0.493 \pm 0.082$ & $-0.551 \pm 0.035$ & $-0.519 \pm 0.061$ & $-0.633 \pm 0.034$ & $-0.438 \pm 0.084$ & $-0.507 \pm 0.022$ & $-0.732 \pm 0.047$ & $-0.769 \pm 0.039$ \\
\hline HIP38140 & $0.100 \pm 0.030$ & $-0.110 \pm 0.067$ & $-0.081 \pm 0.038$ & $-0.060 \pm 0.043$ & $-0.061 \pm 0.052$ & $-0.091 \pm 0.039$ & $-0.029 \pm 0.052$ & $-0.120 \pm 0.041$ & $-0.073 \pm 0.038$ & $-0.121 \pm 0.038$ \\
\hline HIP38183 & $0.042 \pm 0.062$ & $-0.023 \pm 0.055$ & $-0.055 \pm 0.021$ & $-0.015 \pm 0.050$ & $-0.070 \pm 0.134$ & $-0.073 \pm 0.031$ & $-0.012 \pm 0.075$ & $-0.059 \pm 0.059$ & $-0.088 \pm 0.029$ & $-0.118 \pm 0.040$ \\
\hline HIP38267 & $-0.019 \pm 0.111$ & $0.025 \pm 0.051$ & $0.086 \pm 0.028$ & $0.073 \pm 0.073$ & $-0.097 \pm 0.053$ & $-0.002 \pm 0.052$ & $-0.009 \pm 0.080$ & $-0.011 \pm 0.060$ & $-0.087 \pm 0.067$ & $-0.063 \pm 0.060$ \\
\hline HIP38303 & $0.131 \pm 0.071$ & $0.138 \pm 0.084$ & $0.031 \pm 0.060$ & $0.002 \pm 0.064$ & $-0.018 \pm 0.048$ & $-0.017 \pm 0.025$ & $-0.003 \pm 0.074$ & $-0.060 \pm 0.079$ & $-0.092 \pm 0.052$ & $-0.120 \pm 0.045$ \\
\hline HIP38375 & $0.248 \pm 0.029$ & $0.139 \pm 0.088$ & $0.118 \pm 0.035$ & $0.150 \pm 0.109$ & $0.089 \pm 0.061$ & $0.056 \pm 0.055$ & $0.074 \pm 0.081$ & $0.009 \pm 0.048$ & $0.086 \pm 0.038$ & $0.056 \pm 0.052$ \\
\hline HIP38748 & $-0.241 \pm 0.041$ & $-0.078 \pm 0.024$ & $-0.112 \pm 0.028$ & $-0.160 \pm 0.088$ & $-0.210 \pm 0.061$ & $-0.194 \pm 0.010$ & $-0.160 \pm 0.066$ & $-0.193 \pm 0.044$ & $-0.304 \pm 0.032$ & $-0.304 \pm 0.034$ \\
\hline HIP38908 & $-0.272 \pm 0.018$ & $-0.056 \pm 0.042$ & $-0.097 \pm 0.024$ & $-0.146 \pm 0.028$ & $-0.179 \pm 0.055$ & $-0.110 \pm 0.035$ & $-0.072 \pm 0.039$ & $-0.075 \pm 0.008$ & $-0.309 \pm 0.036$ & $-0.276 \pm 0.020$ \\
\hline HIP40693 & $-0.082 \pm 0.015$ & $-0.007 \pm 0.035$ & $0.006 \pm 0.022$ & $-0.059 \pm 0.043$ & $-0.057 \pm 0.067$ & $-0.063 \pm 0.095$ & $0.004 \pm 0.024$ & $-0.094 \pm 0.022$ & $-0.032 \pm 0.050$ & $-0.048 \pm 0.027$ \\
\hline HIP41926 & $-0.392 \pm 0.038$ & $-0.313 \pm 0.056$ & $-0.291 \pm 0.052$ & $-0.381 \pm 0.023$ & $-0.367 \pm 0.051$ & $-0.403 \pm 0.041$ & $-0.275 \pm 0.034$ & $-0.415 \pm 0.045$ & $-0.396 \pm 0.044$ & $-0.437 \pm 0.026$ \\
\hline HIP42808 & $-0.210 \pm 0.029$ & $-0.162 \pm 0.040$ & $-0.108 \pm 0.037$ & $-0.032 \pm 0.046$ & $-0.029 \pm 0.105$ & $-0.138 \pm 0.067$ & $-0.023 \pm 0.075$ & $-0.118 \pm 0.086$ & $-0.047 \pm 0.053$ & $-0.116 \pm 0.046$ \\
\hline HIP45002 & $-0.116 \pm 0.063$ & $0.042 \pm 0.064$ & $-0.006 \pm 0.029$ & $-0.074 \pm 0.061$ & $-0.145 \pm 0.051$ & $-0.069 \pm 0.016$ & $-0.089 \pm 0.071$ & $-0.119 \pm 0.053$ & $-0.201 \pm 0.063$ & $-0.202 \pm 0.046$ \\
\hline HIP46543 & $-0.340 \pm 0.023$ & $-0.255 \pm 0.027$ & $-0.255 \pm 0.035$ & $-0.292 \pm 0.026$ & $-0.299 \pm 0.057$ & $-0.270 \pm 0.020$ & $-0.248 \pm 0.041$ & $-0.265 \pm 0.016$ & $-0.370 \pm 0.030$ & $-0.376 \pm 0.024$ \\
\hline HIP49844 & $0.407 \pm 0.078$ & $0.341 \pm 0.062$ & $0.528 \pm 0.052$ & $0.337 \pm 0.101$ & $0.132 \pm 0.094$ & $0.246 \pm 0.122$ & $0.249 \pm 0.141$ & $0.046 \pm 0.131$ & $0.179 \pm 0.082$ & $0.260 \pm 0.120$ \\
\hline HIP50887 & $0.000 \pm 0.051$ & $0.014 \pm 0.068$ & $0.022 \pm 0.022$ & $0.027 \pm 0.036$ & $0.019 \pm 0.060$ & $0.003 \pm 0.013$ & $0.038 \pm 0.061$ & $-0.016 \pm 0.036$ & $-0.042 \pm 0.044$ & $-0.058 \pm 0.038$ \\
\hline HIP50903 & $-0.071 \pm 0.071$ & $-0.045 \pm 0.061$ & $-0.066 \pm 0.041$ & $-0.075 \pm 0.058$ & $-0.074 \pm 0.052$ & $-0.075 \pm 0.017$ & $-0.047 \pm 0.066$ & $-0.065 \pm 0.040$ & $-0.093 \pm 0.045$ & $-0.149 \pm 0.041$ \\
\hline HIP50939 & $-0.282 \pm 0.038$ & $0.002 \pm 0.049$ & $-0.083 \pm 0.034$ & $-0.155 \pm 0.041$ & $-0.186 \pm 0.055$ & $-0.200 \pm 0.011$ & $-0.156 \pm 0.076$ & $-0.209 \pm 0.038$ & $-0.275 \pm 0.030$ & $-0.268 \pm 0.035$ \\
\hline HIP52316 & $-0.036 \pm 0.016$ & $0.049 \pm 0.023$ & $0.058 \pm 0.018$ & $0.062 \pm 0.069$ & $-0.039 \pm 0.069$ & $0.024 \pm 0.007$ & $0.030 \pm 0.049$ & $-0.004 \pm 0.029$ & $-0.010 \pm 0.062$ & $-0.004 \pm 0.035$ \\
\hline HIP54863 & $0.162 \pm 0.030$ & $0.279 \pm 0.089$ & $0.278 \pm 0.032$ & $0.160 \pm 0.132$ & $0.066 \pm 0.076$ & $0.100 \pm 0.052$ & $0.100 \pm 0.107$ & $0.019 \pm 0.087$ & $0.052 \pm 0.051$ & $0.088 \pm 0.091$ \\
\hline HIP56452 & $-0.416 \pm 0.030$ & $-0.318 \pm 0.079$ & $-0.285 \pm 0.039$ & $-0.373 \pm 0.021$ & $-0.361 \pm 0.064$ & $-0.387 \pm 0.042$ & $-0.270 \pm 0.036$ & $-0.403 \pm 0.040$ & $-0.393 \pm 0.054$ & $-0.435 \pm 0.027$ \\
\hline HIP56830 & $-0.701 \pm 0.025$ & $-0.415 \pm 0.040$ & $-0.445 \pm 0.031$ & $-0.492 \pm 0.024$ & $-0.494 \pm 0.063$ & $-0.538 \pm 0.027$ & $-0.443 \pm 0.041$ & $-0.473 \pm 0.022$ & $-0.770 \pm 0.036$ & $-0.736 \pm 0.023$ \\
\hline HIP57079 & $-0.126 \pm 0.020$ & $-0.077 \pm 0.062$ & $-0.032 \pm 0.024$ & $-0.052 \pm 0.064$ & $-0.062 \pm 0.101$ & $-0.040 \pm 0.012$ & $-0.054 \pm 0.068$ & $-0.028 \pm 0.034$ & $-0.168 \pm 0.031$ & $-0.131 \pm 0.056$ \\
\hline HIP57137 & $-0.155 \pm 0.018$ & $0.016 \pm 0.031$ & $-0.058 \pm 0.018$ & $-0.108 \pm 0.053$ & $-0.091 \pm 0.057$ & $-0.126 \pm 0.010$ & $-0.046 \pm 0.059$ & $-0.138 \pm 0.023$ & $-0.103 \pm 0.034$ & $-0.139 \pm 0.038$ \\
\hline HIP57443 & $-0.341 \pm 0.015$ & $-0.158 \pm 0.056$ & $-0.161 \pm 0.021$ & $-0.241 \pm 0.030$ & $-0.261 \pm 0.074$ & $-0.229 \pm 0.041$ & $-0.177 \pm 0.030$ & $-0.223 \pm 0.015$ & $-0.312 \pm 0.032$ & $-0.323 \pm 0.021$ \\
\hline HIP62103 & $-0.389 \pm 0.039$ & $-0.283 \pm 0.045$ & $-0.290 \pm 0.028$ & $-0.344 \pm 0.019$ & $-0.321 \pm 0.063$ & $-0.307 \pm 0.021$ & $-0.317 \pm 0.056$ & $-0.316 \pm 0.026$ & $-0.422 \pm 0.033$ & $-0.415 \pm 0.027$ \\
\hline HIP62268 & $0.189 \pm 0.021$ & $0.127 \pm 0.063$ & $0.220 \pm 0.022$ & $0.224 \pm 0.090$ & $0.016 \pm 0.071$ & $0.121 \pm 0.038$ & $0.102 \pm 0.081$ & $0.022 \pm 0.062$ & $0.075 \pm 0.064$ & $0.111 \pm 0.056$ \\
\hline HIP62325 & $-0.085 \pm 0.044$ & $0.066 \pm 0.045$ & $0.116 \pm 0.048$ & $0.083 \pm 0.101$ & $-0.028 \pm 0.059$ & $0.049 \pm 0.019$ & $0.063 \pm 0.064$ & $0.030 \pm 0.081$ & $-0.072 \pm 0.060$ & $-0.055 \pm 0.046$ \\
\hline HIP64725 & $0.007 \pm 0.070$ & $0.096 \pm 0.054$ & $0.161 \pm 0.028$ & $0.166 \pm 0.105$ & $0.030 \pm 0.078$ & $0.020 \pm 0.033$ & $0.097 \pm 0.076$ & $0.013 \pm 0.057$ & $-0.017 \pm 0.043$ & $0.023 \pm 0.044$ \\
\hline HIP64751 & $-0.091 \pm 0.066$ & $-0.105 \pm 0.065$ & $-0.070 \pm 0.031$ & $-0.107 \pm 0.075$ & $-0.108 \pm 0.123$ & $-0.115 \pm 0.036$ & $-0.086 \pm 0.086$ & $-0.113 \pm 0.054$ & $-0.193 \pm 0.039$ & $-0.199 \pm 0.037$ \\
\hline HIP64924 & $-0.086 \pm 0.016$ & $0.000 \pm 0.016$ & $0.040 \pm 0.021$ & $-0.001 \pm 0.052$ & $0.001 \pm 0.110$ & $0.033 \pm 0.025$ & $0.026 \pm 0.019$ & $-0.007 \pm 0.021$ & $-0.011 \pm 0.031$ & $-0.022 \pm 0.025$ \\
\hline HIP67851 & $0.063 \pm 0.035$ & $0.286 \pm 0.050$ & $0.298 \pm 0.043$ & $0.137 \pm 0.065$ & $0.040 \pm 0.069$ & $0.220 \pm 0.053$ & $0.190 \pm 0.081$ & $0.143 \pm 0.059$ & $-0.007 \pm 0.038$ & $0.046 \pm 0.065$ \\
\hline HIP67890 & $0.351 \pm 0.034$ & $0.379 \pm 0.056$ & $0.450 \pm 0.044$ & $0.439 \pm 0.143$ & $0.165 \pm 0.074$ & $0.317 \pm 0.074$ & $0.231 \pm 0.096$ & $0.173 \pm 0.077$ & $0.166 \pm 0.056$ & $0.325 \pm 0.096$ \\
\hline HIP70038 & $0.190 \pm 0.067$ & $0.013 \pm 0.086$ & $0.044 \pm 0.024$ & $0.051 \pm 0.061$ & $0.065 \pm 0.046$ & $0.016 \pm 0.021$ & $0.033 \pm 0.067$ & $-0.062 \pm 0.058$ & $0.030 \pm 0.038$ & $-0.007 \pm 0.044$ \\
\hline
\end{tabular}


A\&A 629, A80 (2019)

Table B.3. continued.

\begin{tabular}{|c|c|c|c|c|c|c|c|c|c|c|}
\hline Star & {$[\mathrm{NaI} / \mathrm{H}]$} & {$[\mathrm{MgI} / \mathrm{H}]$} & {$[\mathrm{AlI} / \mathrm{H}]$} & {$[\mathrm{SiI} / \mathrm{H}]$} & {$[\mathrm{CaI} / \mathrm{H}]$} & {$[\mathrm{ScII} / \mathrm{H}]$} & {$[\mathrm{TiI} / \mathrm{H}]$} & {$[\mathrm{TiII} / \mathrm{H}]$} & {$[\mathrm{CrI} / \mathrm{H}]$} & {$[\mathrm{NiI} / \mathrm{H}]$} \\
\hline HIP70336 & $0.317 \pm 0.086$ & $0.058 \pm 0.067$ & $0.117 \pm 0.025$ & $0.221 \pm 0.064$ & $0.087 \pm 0.060$ & $0.132 \pm 0.032$ & $0.099 \pm 0.069$ & $0.031 \pm 0.054$ & $0.078 \pm 0.044$ & $0.112 \pm 0.060$ \\
\hline HIP73927 & $-0.110 \pm 0.054$ & $0.008 \pm 0.057$ & $0.129 \pm 0.021$ & $0.061 \pm 0.064$ & $-0.038 \pm 0.063$ & $0.010 \pm 0.036$ & $0.019 \pm 0.089$ & $-0.056 \pm 0.049$ & $-0.080 \pm 0.055$ & $-0.065 \pm 0.057$ \\
\hline HIP76219 & $0.152 \pm 0.093$ & $0.181 \pm 0.075$ & $0.219 \pm 0.040$ & $0.127 \pm 0.090$ & $-0.005 \pm 0.064$ & $0.099 \pm 0.023$ & $0.074 \pm 0.066$ & $0.032 \pm 0.055$ & $-0.021 \pm 0.041$ & $0.041 \pm 0.049$ \\
\hline HIP77257 & $-0.031 \pm 0.015$ & $0.037 \pm 0.015$ & $0.056 \pm 0.020$ & $0.024 \pm 0.037$ & $0.025 \pm 0.068$ & $0.069 \pm 0.038$ & $0.032 \pm 0.021$ & $0.032 \pm 0.011$ & $-0.026 \pm 0.024$ & $0.016 \pm 0.019$ \\
\hline HIP77512 & $-0.074 \pm 0.026$ & $-0.120 \pm 0.060$ & $0.002 \pm 0.128$ & $-0.095 \pm 0.063$ & $-0.065 \pm 0.050$ & $-0.131 \pm 0.027$ & $-0.058 \pm 0.054$ & $-0.144 \pm 0.032$ & $-0.138 \pm 0.026$ & $-0.163 \pm 0.030$ \\
\hline HIP79137 & $0.118 \pm 0.016$ & $0.248 \pm 0.015$ & $0.274 \pm 0.017$ & $0.180 \pm 0.068$ & $0.123 \pm 0.068$ & $0.221 \pm 0.047$ & $0.251 \pm 0.076$ & $0.141 \pm 0.045$ & $0.135 \pm 0.041$ & $0.137 \pm 0.055$ \\
\hline HIP79522 & $-0.124 \pm 0.018$ & $0.022 \pm 0.056$ & $-0.079 \pm 0.017$ & $-0.082 \pm 0.057$ & $-0.124 \pm 0.052$ & $-0.166 \pm 0.015$ & $-0.111 \pm 0.075$ & $-0.159 \pm 0.053$ & $-0.196 \pm 0.030$ & $-0.202 \pm 0.033$ \\
\hline HIP79605 & $0.159 \pm 0.055$ & $0.102 \pm 0.057$ & $0.239 \pm 0.020$ & $0.233 \pm 0.140$ & $0.054 \pm 0.057$ & $0.139 \pm 0.062$ & $0.098 \pm 0.084$ & $0.023 \pm 0.048$ & $0.045 \pm 0.061$ & $0.091 \pm 0.068$ \\
\hline HIP80910 & $0.308 \pm 0.039$ & $0.316 \pm 0.065$ & $0.292 \pm 0.038$ & $0.215 \pm 0.095$ & $0.101 \pm 0.098$ & $0.257 \pm 0.073$ & $0.192 \pm 0.092$ & $0.084 \pm 0.068$ & $0.118 \pm 0.076$ & $0.205 \pm 0.074$ \\
\hline HIP81300 & $-0.039 \pm 0.020$ & $-0.022 \pm 0.058$ & $0.035 \pm 0.021$ & $-0.007 \pm 0.053$ & $0.056 \pm 0.104$ & $-0.017 \pm 0.025$ & $0.061 \pm 0.036$ & $-0.054 \pm 0.023$ & $0.061 \pm 0.082$ & $-0.011 \pm 0.030$ \\
\hline HIP82989 & $-0.012 \pm 0.017$ & $0.169 \pm 0.043$ & $0.106 \pm 0.019$ & $0.110 \pm 0.043$ & $0.032 \pm 0.065$ & $0.074 \pm 0.016$ & $0.110 \pm 0.079$ & $0.050 \pm 0.039$ & $0.025 \pm 0.027$ & $0.026 \pm 0.045$ \\
\hline HIP83541 & $0.394 \pm 0.015$ & $0.360 \pm 0.068$ & $0.407 \pm 0.017$ & $0.367 \pm 0.065$ & $0.234 \pm 0.053$ & $0.385 \pm 0.064$ & $0.365 \pm 0.041$ & $0.283 \pm 0.064$ & $0.315 \pm 0.034$ & $0.367 \pm 0.038$ \\
\hline HIP83688 & $0.049 \pm 0.022$ & $-0.017 \pm 0.055$ & $0.006 \pm 0.026$ & $0.024 \pm 0.059$ & $0.026 \pm 0.093$ & $0.036 \pm 0.052$ & $0.064 \pm 0.060$ & $0.028 \pm 0.065$ & $0.007 \pm 0.035$ & $-0.053 \pm 0.044$ \\
\hline HIP84478 & $-0.381 \pm 0.053$ & $-0.313 \pm 0.087$ & $-0.323 \pm 0.040$ & $-0.277 \pm 0.062$ & $-0.236 \pm 0.091$ & $-0.439 \pm 0.072$ & $-0.133 \pm 0.069$ & $-0.129 \pm 0.111$ & $-0.276 \pm 0.098$ & $-0.327 \pm 0.084$ \\
\hline HIP85207 & $0.284 \pm 0.062$ & $0.043 \pm 0.078$ & $0.110 \pm 0.033$ & $0.137 \pm 0.064$ & $0.096 \pm 0.060$ & $0.042 \pm 0.020$ & $0.111 \pm 0.081$ & $-0.028 \pm 0.083$ & $0.103 \pm 0.042$ & $0.060 \pm 0.069$ \\
\hline HIP86400 & $-0.059 \pm 0.058$ & $-0.092 \pm 0.063$ & $0.019 \pm 0.052$ & $-0.035 \pm 0.142$ & $-0.096 \pm 0.112$ & $-0.146 \pm 0.087$ & $0.020 \pm 0.078$ & $-0.101 \pm 0.071$ & $-0.025 \pm 0.094$ & $-0.058 \pm 0.052$ \\
\hline HIP86796 & $0.364 \pm 0.020$ & $0.326 \pm 0.027$ & $0.334 \pm 0.028$ & $0.297 \pm 0.060$ & $0.220 \pm 0.050$ & $0.343 \pm 0.048$ & $0.311 \pm 0.028$ & $0.302 \pm 0.044$ & $0.294 \pm 0.026$ & $0.344 \pm 0.028$ \\
\hline HIP87540 & $-0.106 \pm 0.035$ & $-0.008 \pm 0.060$ & $0.032 \pm 0.035$ & $0.027 \pm 0.091$ & $-0.108 \pm 0.052$ & $-0.062 \pm 0.069$ & $-0.079 \pm 0.097$ & $-0.144 \pm 0.060$ & $-0.127 \pm 0.062$ & $-0.109 \pm 0.044$ \\
\hline HIP87834 & $0.228 \pm 0.020$ & $0.293 \pm 0.039$ & $0.361 \pm 0.031$ & $0.266 \pm 0.097$ & $0.134 \pm 0.060$ & $0.190 \pm 0.036$ & $0.228 \pm 0.073$ & $0.125 \pm 0.063$ & $0.163 \pm 0.072$ & $0.192 \pm 0.065$ \\
\hline HIP89962 & $-0.167 \pm 0.022$ & $-0.097 \pm 0.036$ & $-0.072 \pm 0.018$ & $-0.086 \pm 0.046$ & $-0.119 \pm 0.049$ & $-0.116 \pm 0.019$ & $-0.098 \pm 0.067$ & $-0.107 \pm 0.031$ & $-0.176 \pm 0.084$ & $-0.184 \pm 0.033$ \\
\hline HIP94521 & $0.086 \pm 0.076$ & $0.093 \pm 0.054$ & $0.065 \pm 0.025$ & $0.037 \pm 0.074$ & $-0.051 \pm 0.052$ & $-0.015 \pm 0.032$ & $-0.024 \pm 0.069$ & $-0.084 \pm 0.035$ & $-0.090 \pm 0.051$ & $-0.057 \pm 0.045$ \\
\hline HIP95447 & $0.484 \pm 0.048$ & $0.331 \pm 0.060$ & $0.477 \pm 0.024$ & $0.404 \pm 0.061$ & $0.351 \pm 0.102$ & $0.474 \pm 0.050$ & $0.480 \pm 0.053$ & $0.372 \pm 0.049$ & $0.363 \pm 0.026$ & $0.454 \pm 0.050$ \\
\hline HIP96229 & $0.262 \pm 0.026$ & $0.328 \pm 0.061$ & $0.476 \pm 0.025$ & $0.221 \pm 0.134$ & $0.118 \pm 0.059$ & $0.329 \pm 0.077$ & $0.260 \pm 0.135$ & $0.128 \pm 0.083$ & $0.109 \pm 0.052$ & $0.148 \pm 0.102$ \\
\hline HIP98066 & $-0.066 \pm 0.021$ & $0.009 \pm 0.045$ & $0.009 \pm 0.023$ & $0.011 \pm 0.027$ & $0.024 \pm 0.066$ & $0.049 \pm 0.031$ & $0.044 \pm 0.030$ & $0.043 \pm 0.030$ & $0.003 \pm 0.025$ & $-0.012 \pm 0.032$ \\
\hline HIP98767 & $0.254 \pm 0.022$ & $0.374 \pm 0.055$ & $0.350 \pm 0.024$ & $0.279 \pm 0.052$ & $0.223 \pm 0.070$ & $0.392 \pm 0.062$ & $0.373 \pm 0.047$ & $0.274 \pm 0.023$ & $0.276 \pm 0.047$ & $0.304 \pm 0.049$ \\
\hline HIP98920 & $0.108 \pm 0.022$ & $0.177 \pm 0.065$ & $0.190 \pm 0.023$ & $0.140 \pm 0.087$ & $0.022 \pm 0.050$ & $0.103 \pm 0.049$ & $0.079 \pm 0.089$ & $0.004 \pm 0.057$ & $0.003 \pm 0.046$ & $0.053 \pm 0.053$ \\
\hline HIP99171 & $0.070 \pm 0.018$ & $0.222 \pm 0.037$ & $0.254 \pm 0.019$ & $0.146 \pm 0.097$ & $0.076 \pm 0.061$ & $0.075 \pm 0.035$ & $0.188 \pm 0.076$ & $0.048 \pm 0.048$ & $-0.007 \pm 0.077$ & $0.013 \pm 0.052$ \\
\hline HIP99461 & $-0.499 \pm 0.022$ & $-0.293 \pm 0.082$ & $-0.243 \pm 0.026$ & $-0.412 \pm 0.047$ & $-0.367 \pm 0.085$ & $-0.458 \pm 0.053$ & $-0.169 \pm 0.048$ & $-0.424 \pm 0.042$ & $-0.493 \pm 0.076$ & $-0.570 \pm 0.030$ \\
\hline HIP99825 & $0.031 \pm 0.011$ & $0.007 \pm 0.026$ & $0.052 \pm 0.018$ & $-0.018 \pm 0.054$ & $-0.022 \pm 0.106$ & $0.038 \pm 0.065$ & $0.098 \pm 0.081$ & $-0.024 \pm 0.080$ & $0.029 \pm 0.067$ & $0.016 \pm 0.051$ \\
\hline HIP99894 & $-0.247 \pm 0.021$ & $-0.191 \pm 0.033$ & $-0.144 \pm 0.021$ & $-0.143 \pm 0.028$ & $-0.113 \pm 0.084$ & $-0.110 \pm 0.011$ & $-0.101 \pm 0.063$ & $-0.138 \pm 0.022$ & $-0.174 \pm 0.068$ & $-0.211 \pm 0.041$ \\
\hline HIP99913 & $0.081 \pm 0.036$ & $0.153 \pm 0.063$ & $0.167 \pm 0.022$ & $0.194 \pm 0.094$ & $0.050 \pm 0.063$ & $0.134 \pm 0.037$ & $0.136 \pm 0.087$ & $0.093 \pm 0.065$ & $0.072 \pm 0.056$ & $0.107 \pm 0.054$ \\
\hline HIP101221 & $-0.164 \pm 0.022$ & $-0.368 \pm 0.053$ & $-0.154 \pm 0.025$ & $-0.100 \pm 0.093$ & $-0.234 \pm 0.067$ & $-0.241 \pm 0.047$ & $-0.224 \pm 0.061$ & $-0.269 \pm 0.081$ & $-0.250 \pm 0.091$ & $-0.152 \pm 0.080$ \\
\hline HIP101345 & $-0.028 \pm 0.016$ & $0.107 \pm 0.014$ & $0.094 \pm 0.018$ & $0.068 \pm 0.039$ & $0.057 \pm 0.071$ & $0.121 \pm 0.059$ & $0.082 \pm 0.023$ & $0.090 \pm 0.012$ & $0.051 \pm 0.026$ & $0.055 \pm 0.029$ \\
\hline HIP101916 & $-0.002 \pm 0.022$ & $0.061 \pm 0.029$ & $0.035 \pm 0.034$ & $0.078 \pm 0.062$ & $0.062 \pm 0.067$ & $0.086 \pm 0.020$ & $0.062 \pm 0.039$ & $0.079 \pm 0.023$ & $0.038 \pm 0.025$ & $0.036 \pm 0.012$ \\
\hline HIP102891 & $-0.188 \pm 0.017$ & $-0.074 \pm 0.041$ & $-0.021 \pm 0.022$ & $-0.057 \pm 0.061$ & $-0.207 \pm 0.052$ & $-0.141 \pm 0.047$ & $-0.122 \pm 0.088$ & $-0.187 \pm 0.056$ & $-0.230 \pm 0.063$ & $-0.196 \pm 0.050$ \\
\hline HIP107649 & $-0.110 \pm 0.023$ & $-0.010 \pm 0.064$ & $-0.030 \pm 0.032$ & $-0.024 \pm 0.032$ & $0.000 \pm 0.046$ & $0.012 \pm 0.012$ & $0.014 \pm 0.029$ & $-0.007 \pm 0.013$ & $-0.020 \pm 0.028$ & $-0.041 \pm 0.017$ \\
\hline HIP108513 & $0.249 \pm 0.032$ & $0.304 \pm 0.077$ & $0.267 \pm 0.038$ & $0.312 \pm 0.108$ & $0.127 \pm 0.059$ & $0.140 \pm 0.072$ & $0.202 \pm 0.078$ & $0.056 \pm 0.065$ & $0.150 \pm 0.041$ & $0.212 \pm 0.058$ \\
\hline HIP108868 & $0.324 \pm 0.023$ & $0.235 \pm 0.059$ & $0.196 \pm 0.025$ & $0.195 \pm 0.060$ & $0.058 \pm 0.063$ & $0.132 \pm 0.030$ & $0.113 \pm 0.083$ & $-0.005 \pm 0.084$ & $0.048 \pm 0.038$ & $0.090 \pm 0.051$ \\
\hline HIP 108870 & $-0.198 \pm 0.041$ & $-0.131 \pm 0.078$ & $-0.081 \pm 0.019$ & $-0.134 \pm 0.078$ & $-0.142 \pm 0.097$ & $-0.253 \pm 0.103$ & $-0.022 \pm 0.101$ & $-0.091 \pm 0.129$ & $-0.109 \pm 0.087$ & $-0.149 \pm 0.060$ \\
\hline HIP109577 & $0.112 \pm 0.033$ & $0.004 \pm 0.081$ & $0.173 \pm 0.033$ & $0.094 \pm 0.056$ & $0.083 \pm 0.069$ & $0.085 \pm 0.034$ & $0.133 \pm 0.079$ & $0.060 \pm 0.045$ & $0.054 \pm 0.082$ & $0.079 \pm 0.044$ \\
\hline HIP 109822 & $-0.184 \pm 0.013$ & $0.023 \pm 0.030$ & $0.048 \pm 0.015$ & $-0.080 \pm 0.042$ & $-0.103 \pm 0.074$ & $-0.038 \pm 0.074$ & $0.021 \pm 0.088$ & $-0.023 \pm 0.043$ & $-0.234 \pm 0.040$ & $-0.220 \pm 0.036$ \\
\hline HIP110109 & $-0.251 \pm 0.017$ & $-0.168 \pm 0.042$ & $-0.194 \pm 0.030$ & $-0.200 \pm 0.016$ & $-0.227 \pm 0.055$ & $-0.166 \pm 0.022$ & $-0.178 \pm 0.030$ & $-0.167 \pm 0.017$ & $-0.227 \pm 0.026$ & $-0.231 \pm 0.012$ \\
\hline HIP113148 & $0.264 \pm 0.031$ & $0.326 \pm 0.047$ & $0.333 \pm 0.029$ & $0.331 \pm 0.112$ & $0.106 \pm 0.061$ & $0.316 \pm 0.090$ & $0.196 \pm 0.099$ & $0.121 \pm 0.073$ & $0.123 \pm 0.063$ & $0.252 \pm 0.091$ \\
\hline HIP113357 & $0.236 \pm 0.012$ & $0.174 \pm 0.037$ & $0.206 \pm 0.016$ & $0.205 \pm 0.065$ & $0.162 \pm 0.075$ & $0.279 \pm 0.061$ & $0.204 \pm 0.015$ & $0.184 \pm 0.021$ & $0.196 \pm 0.021$ & $0.237 \pm 0.018$ \\
\hline HIP113801 & $-0.113 \pm 0.019$ & $0.037 \pm 0.047$ & $-0.038 \pm 0.025$ & $-0.052 \pm 0.054$ & $-0.081 \pm 0.049$ & $-0.072 \pm 0.014$ & $-0.051 \pm 0.080$ & $-0.072 \pm 0.048$ & $-0.155 \pm 0.043$ & $-0.146 \pm 0.033$ \\
\hline HIP114526 & $-0.127 \pm 0.046$ & $-0.022 \pm 0.050$ & $-0.009 \pm 0.029$ & $0.005 \pm 0.055$ & $-0.028 \pm 0.068$ & $-0.029 \pm 0.016$ & $-0.015 \pm 0.069$ & $-0.027 \pm 0.093$ & $-0.098 \pm 0.051$ & $-0.101 \pm 0.035$ \\
\hline HIP114699 & $-0.170 \pm 0.016$ & $-0.011 \pm 0.035$ & $-0.004 \pm 0.018$ & $-0.097 \pm 0.010$ & $-0.094 \pm 0.070$ & $-0.036 \pm 0.016$ & $0.004 \pm 0.041$ & $-0.046 \pm 0.017$ & $-0.118 \pm 0.045$ & $-0.151 \pm 0.018$ \\
\hline HIP115953 & $0.575 \pm 0.056$ & $0.476 \pm 0.043$ & $0.575 \pm 0.037$ & $0.486 \pm 0.102$ & $0.266 \pm 0.074$ & $0.563 \pm 0.126$ & $0.417 \pm 0.073$ & $0.302 \pm 0.081$ & $0.299 \pm 0.069$ & $0.509 \pm 0.099$ \\
\hline HIP116422 & $0.211 \pm 0.046$ & $0.100 \pm 0.061$ & $0.230 \pm 0.027$ & $0.280 \pm 0.125$ & $0.012 \pm 0.057$ & $0.164 \pm 0.078$ & $0.103 \pm 0.107$ & $-0.003 \pm 0.076$ & $0.031 \pm 0.044$ & $0.100 \pm 0.071$ \\
\hline HIP116624 & $-0.060 \pm 0.036$ & $0.063 \pm 0.047$ & $0.089 \pm 0.025$ & $0.084 \pm 0.080$ & $-0.104 \pm 0.049$ & $-0.031 \pm 0.023$ & $-0.045 \pm 0.101$ & $-0.119 \pm 0.067$ & $-0.126 \pm 0.039$ & $-0.046 \pm 0.061$ \\
\hline
\end{tabular}


S. Hojjatpanah et al.: Catalog for the ESPRESSO blind radial velocity exoplanet survey

Table B.4. $[\mathrm{X} / \mathrm{Fe}]$ abundance ratios for Carbon $(\mathrm{X}=\mathrm{C})$ and Oxygen $(\mathrm{X}=\mathrm{O})$ with errors in measurements of lines for sample of stars with $T_{\text {eff }}>5200 \mathrm{~K}$.

\begin{tabular}{|c|c|c|c|c|}
\hline Star & {$[\mathrm{O} / \mathrm{Fe}]$} & errO & {$[\mathrm{C} / \mathrm{Fe}]$} & errC \\
\hline HIP1599 & 0.069 & 0.090 & -0.072 & 0.037 \\
\hline HIP8102 & 0.288 & 0.101 & 0.188 & 0.089 \\
\hline HIP10138 & 0.176 & .090 & 0.177 & 0.068 \\
\hline HIP13402 & -0.014 & 0.092 & -0.024 & 0.077 \\
\hline HIP15330 & 0.0 & & -0.008 & 0.014 \\
\hline HIP15371 & 0.219 & 0.100 & -0.014 & 0.026 \\
\hline HIP15510 & 99.990 & 0.000 & 0.107 & 0.129 \\
\hline HIP16852 & 0.025 & 0.058 & -0.048 & 0.031 \\
\hline HIP22263 & -0.016 & 113 & -0 . & 0.017 \\
\hline HIP23835 & 0.314 & 0.048 & & 0.014 \\
\hline HIP29271 & 0.050 & 0.080 & -0.050 & 0.015 \\
\hline HIP37606 & 0.016 & 0.0 & -0.076 & 0.018 \\
\hline HIP3 & 99.9 & 0.0 & 0.3 & 0.087 \\
\hline HIP38908 & 0.383 & 0.062 & 0.162 & 0.042 \\
\hline HIP40693 & -0.041 & 0.107 & -0.034 & 0.031 \\
\hline HIP41926 & 99.990 & 0.000 & 0.117 & 0.135 \\
\hline HIP56452 & 0.184 & 0.10 & 0.1 & 0.174 \\
\hline HIP57443 & .200 & .103 & 0.040 & 0.084 \\
\hline HIP64924 & -0.014 & 0.095 & -0.053 & 0.021 \\
\hline HIP77257 & 0.013 & 0.038 & -0.037 & 0.024 \\
\hline HIP81300 & -0.094 & 0.117 & 0.042 & 0.084 \\
\hline HIP8 & -0.113 & 0.0 & -0.093 & 0.049 \\
\hline HIP86796 & -0.039 & 0.048 & -0.063 & 0.028 \\
\hline HIP95447 & -0.153 & 0.063 & -0.162 & 0.042 \\
\hline HIP98066 & -0.100 & 0.065 & -0.125 & 0.027 \\
\hline HIP98767 & -0.081 & 0.0 & -0.083 & 0.027 \\
\hline HIP101345 & 0.020 & 0.052 & -0.063 & 0.014 \\
\hline HIP101916 & -0.027 & 0.049 & -0.122 & 0.037 \\
\hline HIP107649 & -0.114 & 0.081 & -0.126 & 0.034 \\
\hline HIP110109 & 0.020 & & -0.086 & 0.042 \\
\hline HIP113357 & -0.243 & 0.048 & -0.073 & 0.014 \\
\hline HIP114699 & 0.172 & 0.058 & -0.071 & 0.037 \\
\hline
\end{tabular}

Table B.5. 21 spectroscopic identified binary by Pourbaix et al. (2004) in the sample.

\begin{tabular}{ccccc}
\hline \hline Star & RA (hh:mm:ss) & Dec $\left(^{\circ}:^{\prime}:^{\prime \prime}\right)$ & $V$ & Spectral-type \\
\hline HIP4592 & 005845.75 & -055258.1 & 6.59 & K0 \\
HIP11964 & 023422.57 & -434746.9 & 8.70 & K7Ve \\
HIP17207 & 034110.52 & +033641.0 & 9.96 & M0V \\
HIP23221 & 045950.45 & -101548.0 & 5.38 & K0IV \\
HIP25282 & 052428.91 & -005328.8 & 5.08 & G9III-IV \\
HIP27913 & 055422.98 & +201634.2 & 4.41 & G0V \\
HIP29982 & 061840.35 & +090249.9 & 6.24 & G5III \\
HIP34608 & 071006.68 & +211449.1 & 6.43 & G8V \\
HIP39198 & 080103.40 & -062509.1 & 6.46 & G5 \\
HIP45170 & 091217.55 & +145945.7 & 6.51 & G9V \\
HIP55505 & 112205.29 & -244639.8 & 9.41 & K4-5 \\
HIP73182 & 145726.54 & -212441.5 & 8.33 & M1.5V \\
HIP82817 & 165528.75 & -082010.8 & 9.02 & M3Ve \\
HIP86400 & 173916.92 & +033318.9 & 6.52 & K3V \\
HIP90729 & 183041.65 & +204854.2 & 6.50 & G8IV \\
HIP91751 & 184236.12 & -070425.2 & 6.15 & K1III \\
HIP95066 & 192032.91 & -052456.8 & 5.00 & G8III-IV \\
HIP98351 & 195856.43 & -303218.2 & 6.28 & K1III \\
HIP101955 & 203937.71 & +045819.3 & 7.89 & K5V \\
HIP107095 & 214132.86 & -140251.4 & 5.18 & G2IV \\
HIP111802 & 223845.58 & -203716.1 & 9.09 & dM1e \\
\hline
\end{tabular}

